\title{
SOKKIИ'"
}
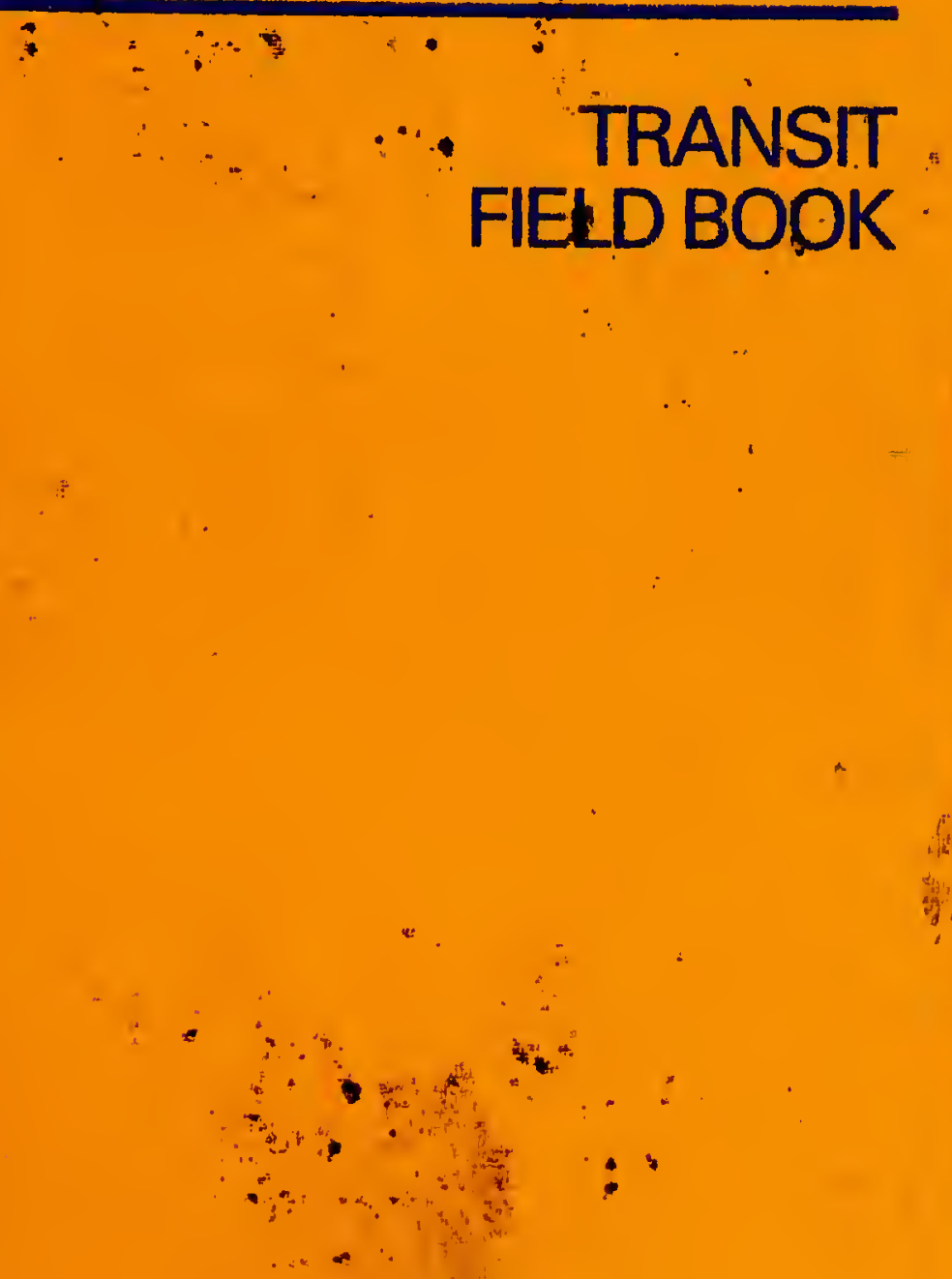

No. 8152-00 
Property of Lee J Hickey

$$
\begin{aligned}
& \text { Address Dpt. of Geolonte reophyses } \\
& \text { yale ciniv }
\end{aligned}
$$

$$
\begin{aligned}
& \text { Telephone } \frac{176 \text { whitey Aver }}{\text { New HAVIN, CT. CG5T! }} \\
& (20 j) 785-86.68
\end{aligned}
$$

This Book is manufactured of a High Grade $50 \%$ Rag Ledger Paper having a Water Resistant Surface, and is sewed with Nylon Waterproof Thread. 


\section{N DEX}


<smiles>[CH]1C=CC=C1</smiles>

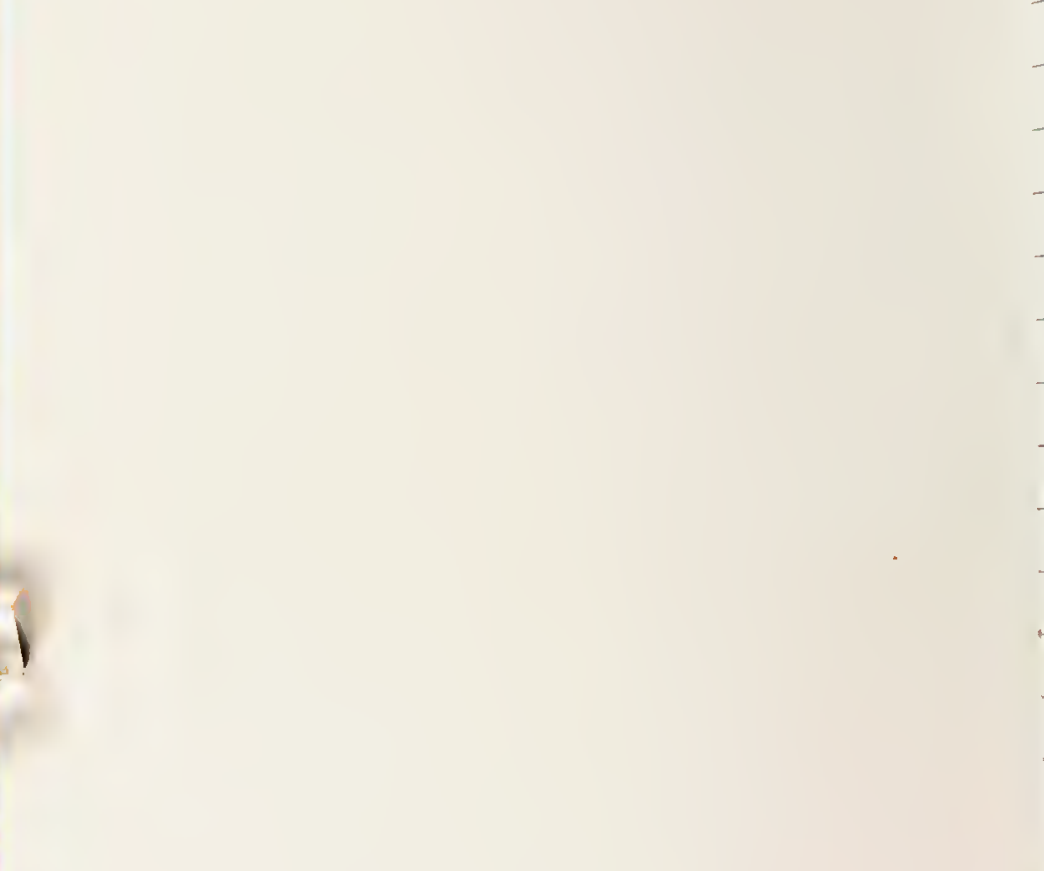




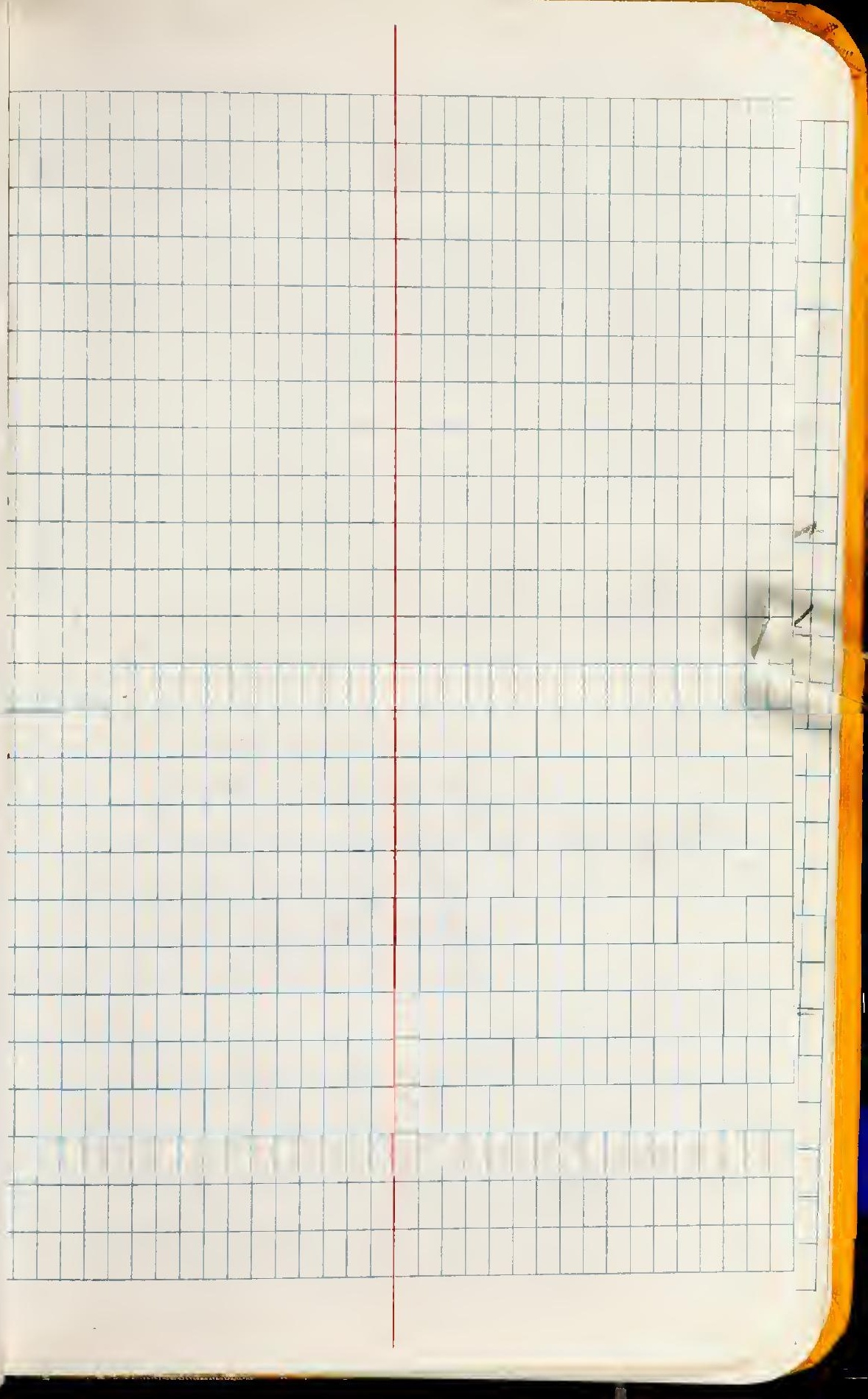


Yorkshire $\quad \mid 9 / 20 / 62$

Scalby Mess Mocki at Scniby Beuk.

$$
\text { SE } 0320.9145
$$

Sheer is. Orfupue Surver-Sarboro

200.9201

w Chris Hill

Geff Hickel

David ThYlor

Scaby Fu - Bajoc/an

Lomg hab mbr (Upper)

Senrburo $\mathrm{km}$.

Gristhurpe Mer

jhebkerstun mbr

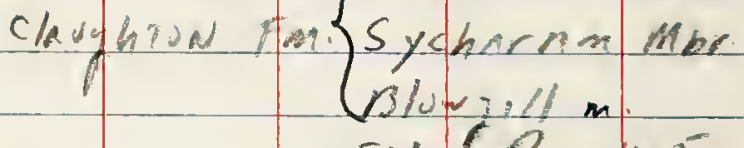

Dle; Beck Fm

$\begin{aligned} \text { Lallowich tm } & \text { Deltelu } \\ & \text { Conl Rs }\end{aligned}$

Dogger

Spe P. $h_{2}$ of Brit. aesh Surver Gullfe to gcol of Eastern Erglpal for sel fiz frem ropatpaye for sectoun 


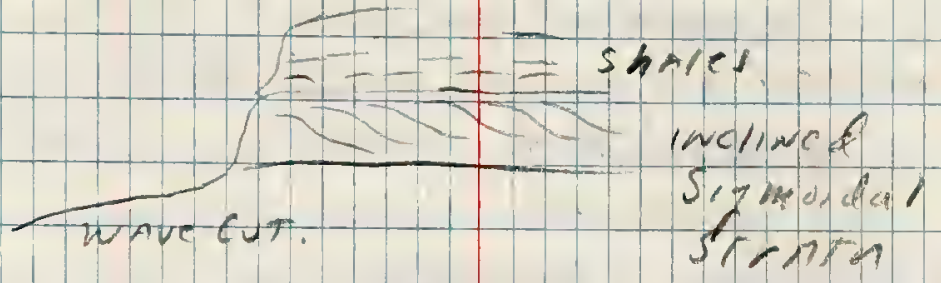

Meander chawnels w

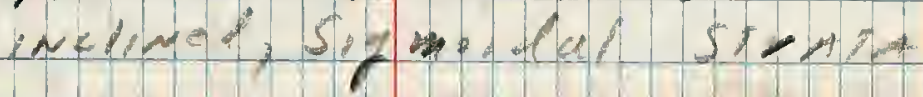

Upper Scalby cubs.5s + lenses 4 muldy SS.

Lu scaldx Fm. - Laterel accreTiul - Channels W/intervenimy c/ays

$$
\text { s.17s }
$$

Locality in Lem Nab ner of SAAlbr fm. 


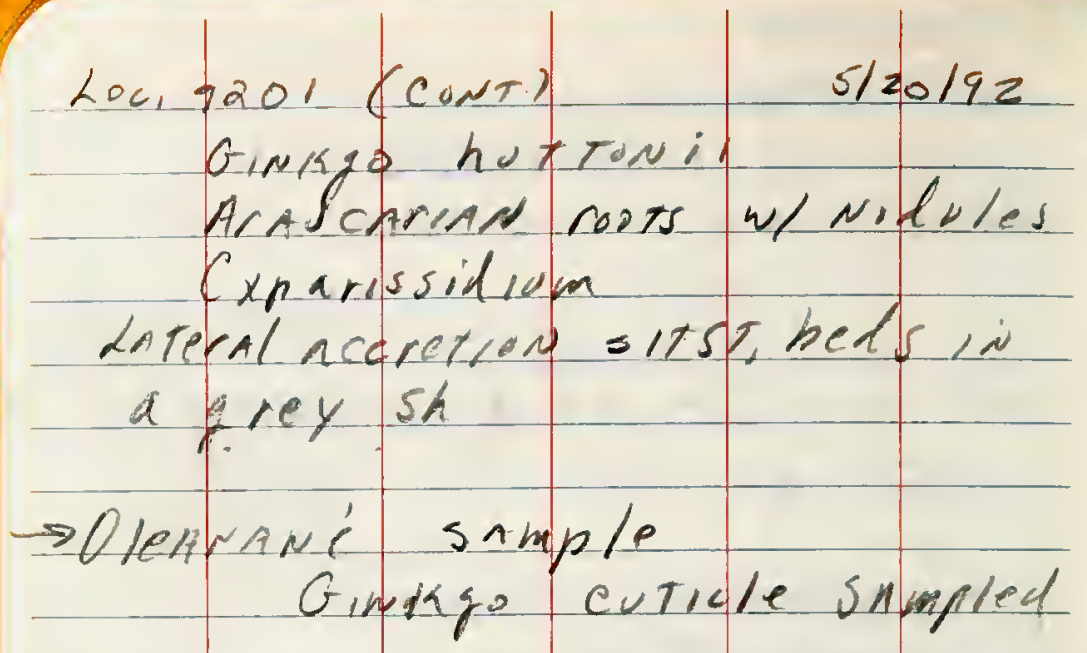

$\rightarrow 920101$. sample frum Gink?0 ned, Tight, very tigr. ss to s/zsi

$9201 \mathrm{C} \gamma 1$. Sample - From s/umpel but Tizht dArk grey Clays of Long wab mbr. of scnlby A. Mulstine

henses of fali ace rmulatioi ss. nierspercel w med quey mulit whin lavers of up to $1 \mathrm{~cm}$ if IT. jores slist.

- Tups ot Ss. chamrels 
Greguently lenckel w/ vert robts a limonte below od

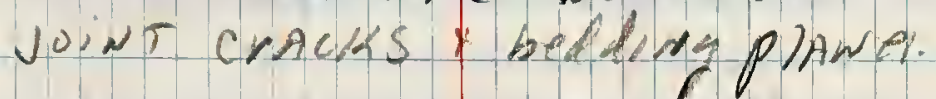

Facies - Menuder belt sarls $r$ flool basin shs.

Lacalnty is on ond Sunw MAp

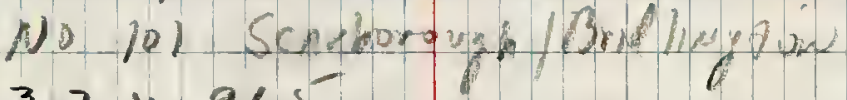
$3.2 \times 91.5$ 


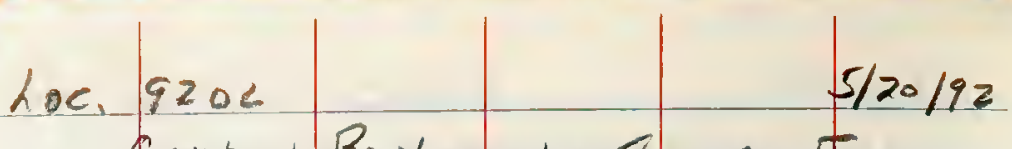

Caytun Bay on the point

is the bie catleal Yon's Yab.

ON. ord Survey maplol at.

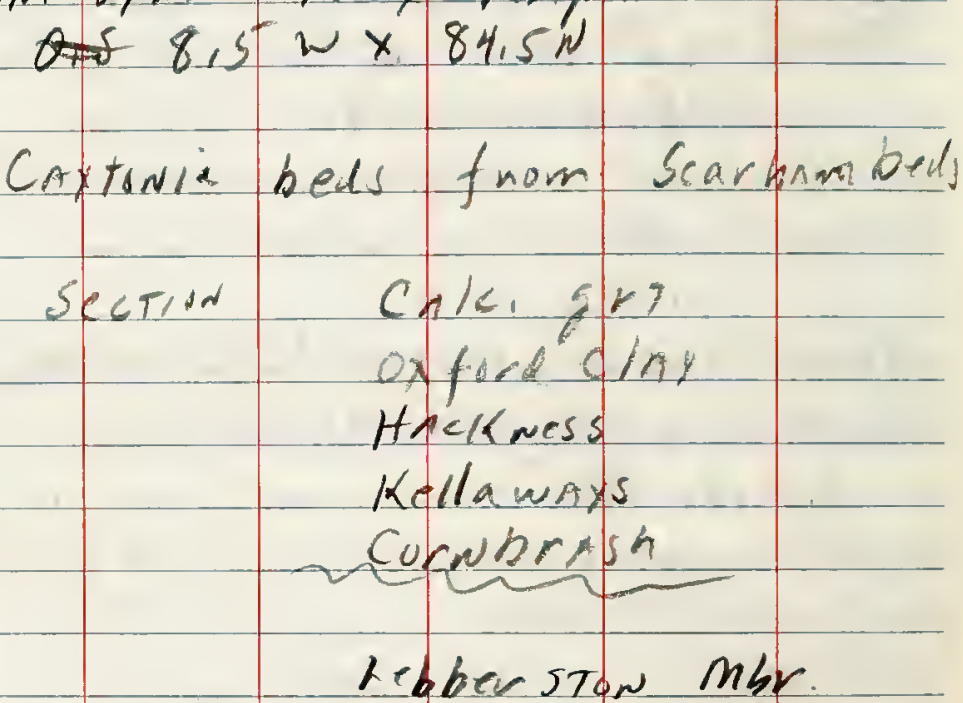

Lebber stow Mbr.

Sycirhams Fun.

Sycarham mbr of The Clayghted Fm. is win-mprists.

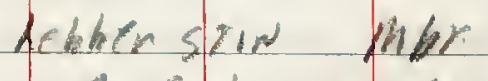

1) Col ew or Arewte

Show $x$ - medeng but This

is u, unll fin

$x$-bxting flat 
$9203 a \quad 5 / 20 / 92$ CAyTON BAy - S. Side

Base of Grislihorpe mor 1 Clavghion Fm

2T. Quey Silty Clay of suplpised now - marive middle deltaic Fm.

Some ironstone nodules present

From 1 m. ahore Top of manime Lebperstor mbr.

92036 Classic CAytonia beds in Middle Gristhorp. mbr of claughtid Fn.

Visited $512019=$ xagow $1 / 22 / 92$ 
92036

Taenropteris smalla large Willsomia

$N_{1}$ lls sonioperis

Cladophlehis dewiculata caytonia fruiss Brachr phyllum Geimitzia sagenapteris Equiserum

Todik dennane Bulk and Taxow Bulk 5 Mmples

Fine grained w/ carbs la miruce forohahly back swamp

Alposit

Laterally probahly Ehawnel Ss units.as IT is interbedted wl them

Chanpeld SS. \$T Tops w/ leachiny to $0.5 \mathrm{~m}$ t reofs (verticit) 
$9203 C$

$5 / 20192$

Caytoria bed about $300 \mathrm{~m}$ S. OT MAIN CAYTONIA $10 \mathrm{c}$

on $N$ side of Yous Nab

Brond fIAT chawmel SSS up To $1 \mathrm{~mm} 1.5 \mathrm{~m}$ Thiek

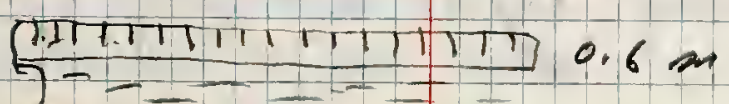

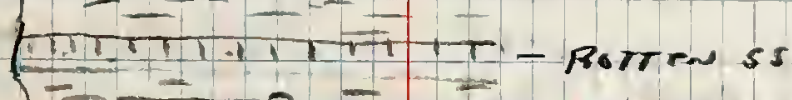

- impin

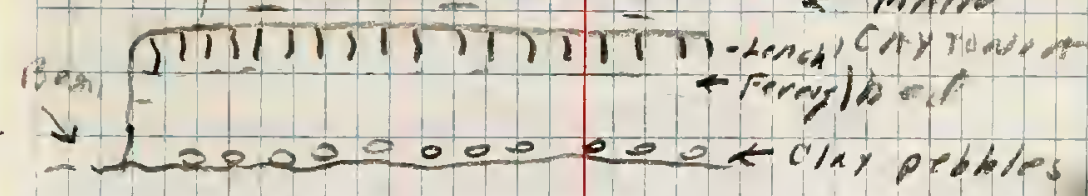

Osmundaceae

Geinitzid appenrs'more abundan

Bevisited $5 / 23 / 92$

Im.

$1111-B 0075$

obob-Fe stive 


$$
9204
$$

Oxford Clay

From the toe af a slump block Inst is about $1 / 4$ mri in berg th

Good Tight sample but a o list nit a Cloy

not CArbONACeOUS. 
yorkshire midale a upper Jurassic segura.

STinges.

Kimmeridge Ciay

V Calcalreues Grit

Coral Bay

onford Clay

hackness Drek

Kellaways Rock

Corwbrash

11111111111

Scalb , tm

Scarborough Fm

Gristhorpe mbr \& CAy

bavenscar Gp. yows wab Fom habbers- Baj

CDelTAiC Seris Millepere bed So Yours min

Ellerbeck Fin

SAfTwich Fm.
K, $\mathrm{mm}$.

ONF

call.

IIII

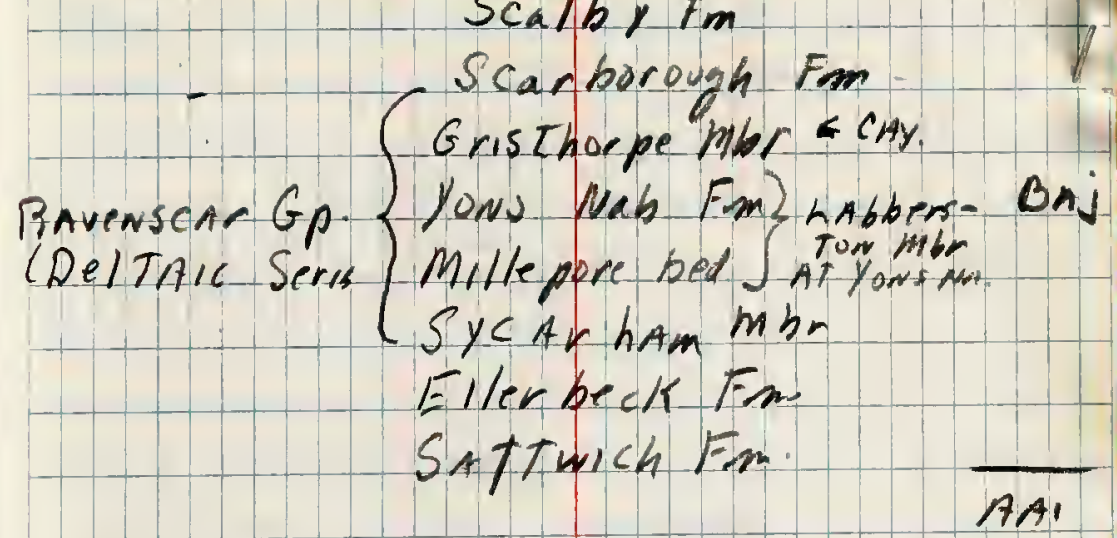




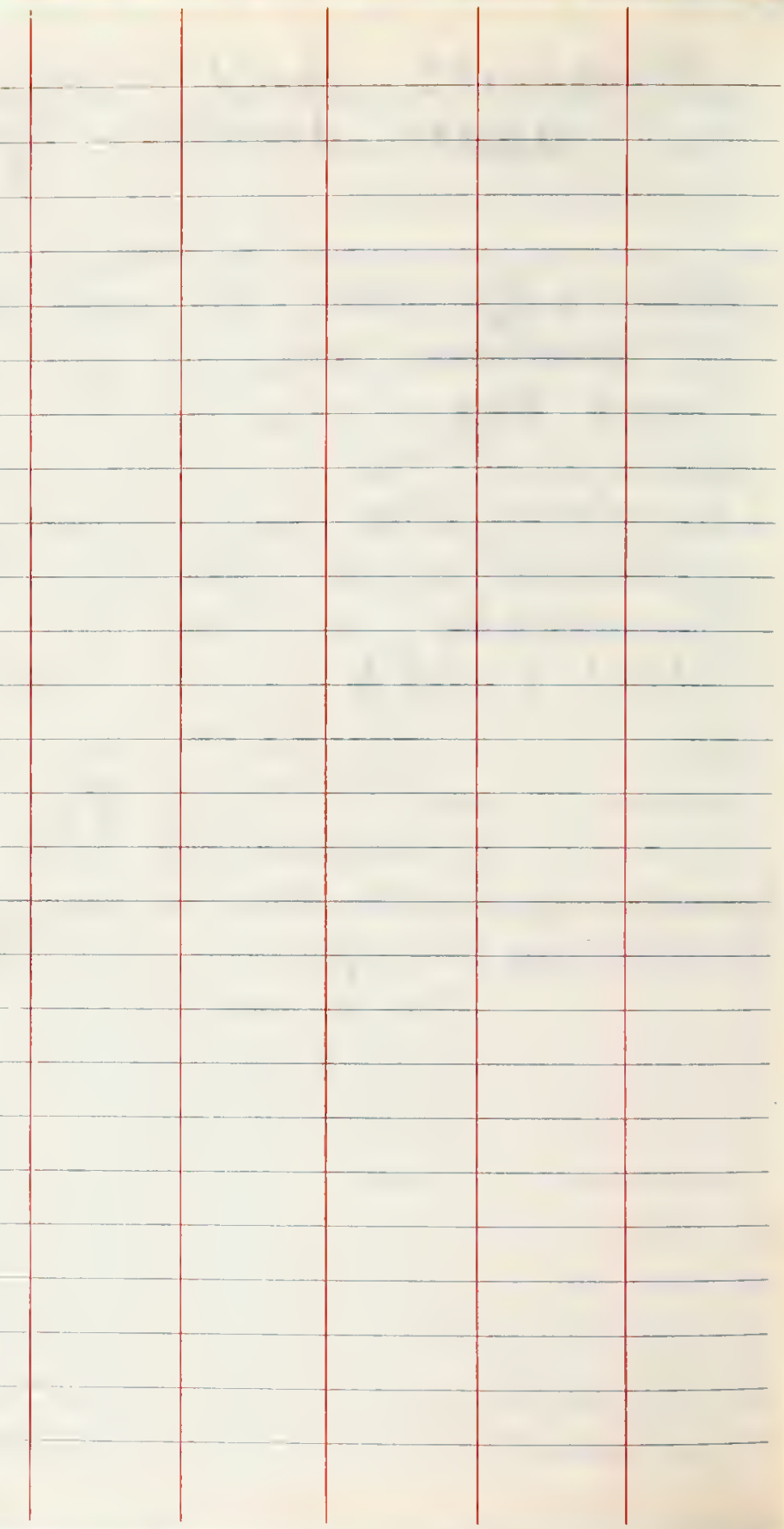

$!$

1
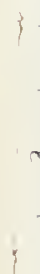
$\pi$
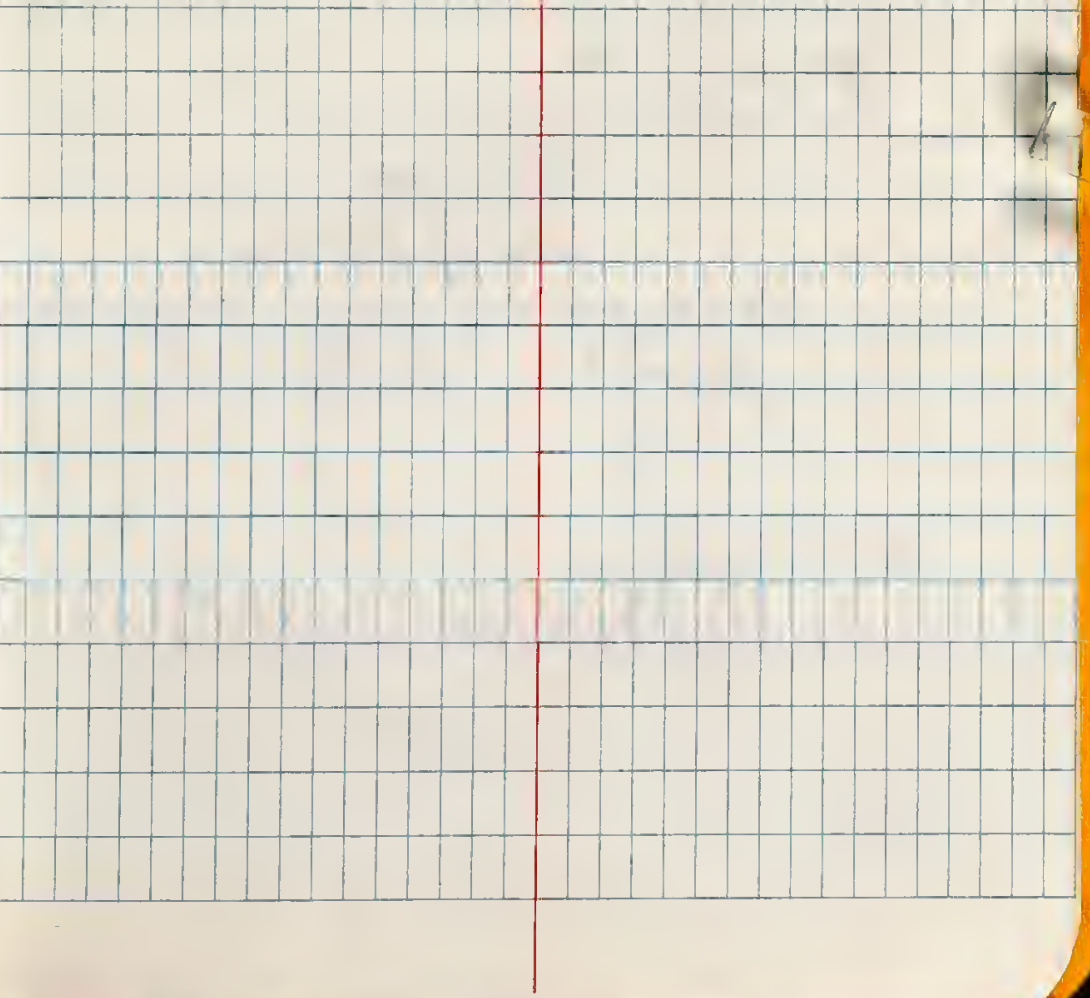


\begin{tabular}{l|l|l|} 
Loc $920 s$ & & $5 / 21 / 92$ \\
Hasty Bank & & \\
Chris Hills & Dissertaleon. \\
Sect. D. &
\end{tabular}

Saltwien Fm. Aalenian

Equistum

Corysuspens.

Pteruma

Dacypteris

Pachypteris has regular pinorste lenf.

Paleocelolyy

Luwer pelta plain 570 mieraplawertur 
Pachypteris

Sagewapteris colpoides

sphemonaieva gyrom

Equistum

PTIlophyllym pectinories.

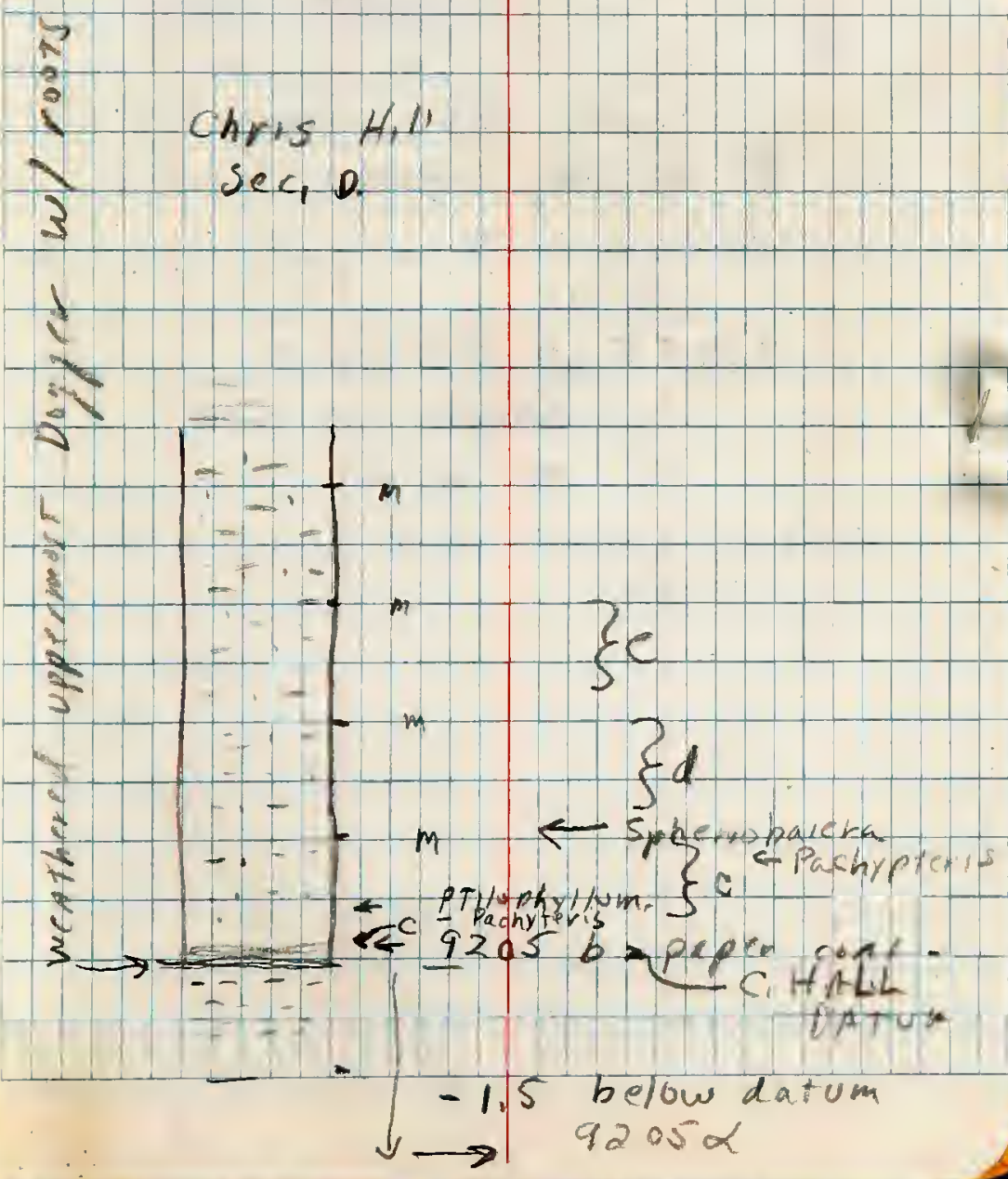




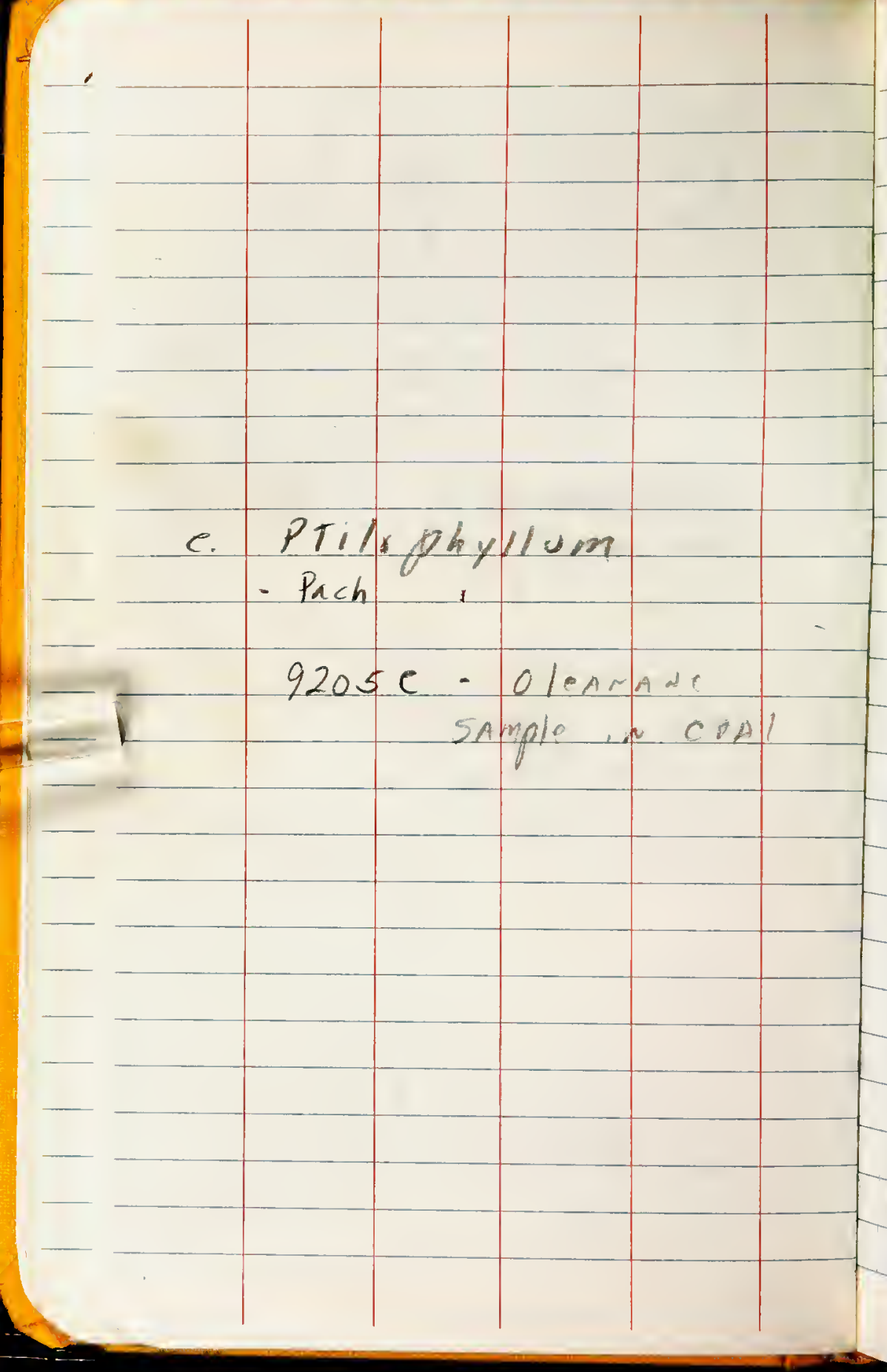


9206 Whithy EAST Cl/fF

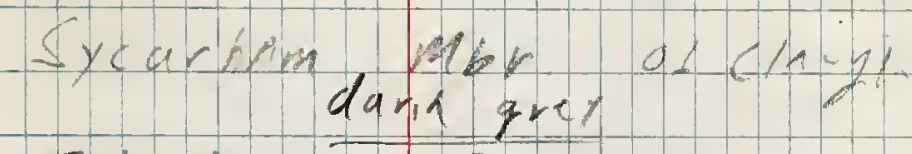

Elperbeck Fun rerroy ss

SALTWICh Fm.

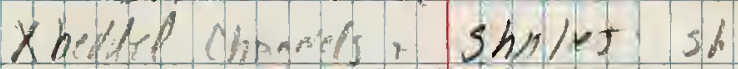

- Im Dogper Thenomnine ss.

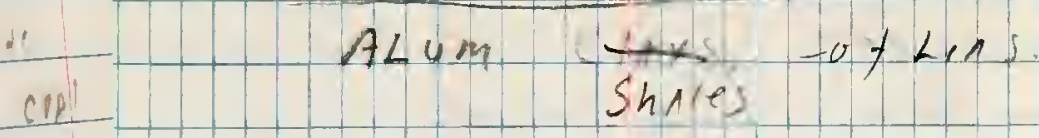

D. 0 D

pos tmy nitw 
5/22/92

9207 Roger Trod (N. Side)

9257 a J $x$ cha cham $0,3 \mathrm{~m}$ abore in Hihes ledye 4

Ferugimears ss w Iberrowy : shells some siferite nilwes Booted t limomite yellow meathered. SS. hecomes more masteve upward.

This Ellerheck, wreval is a marive fdelthic interfingered ". seyuedac

$w$ cycles of ss lobes inat grow swamps andare ther infundeted 
b) marine contras Wail fined by wert than so sheet. Acer ss swamp mu j

Laver delta plane is slight

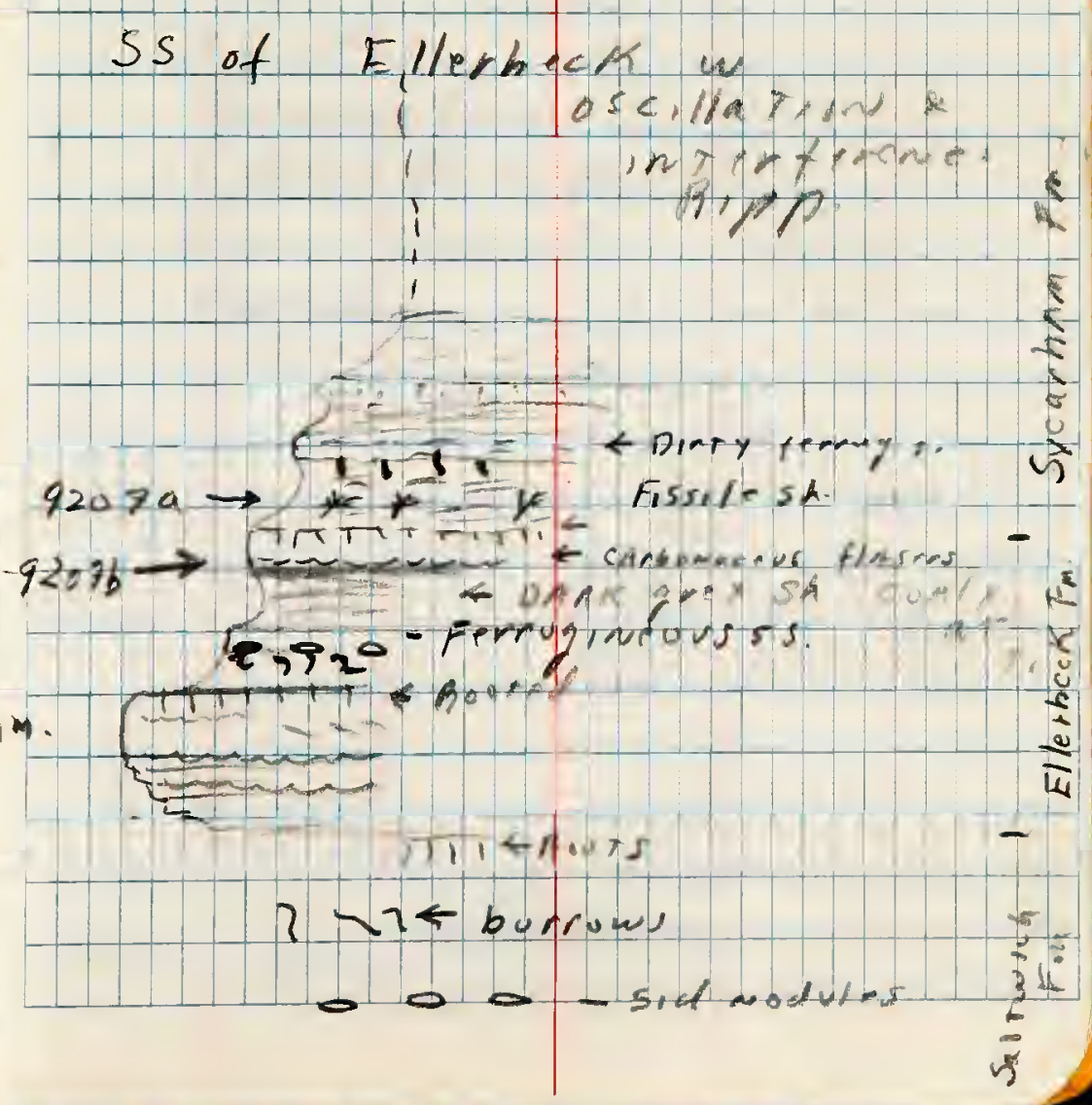




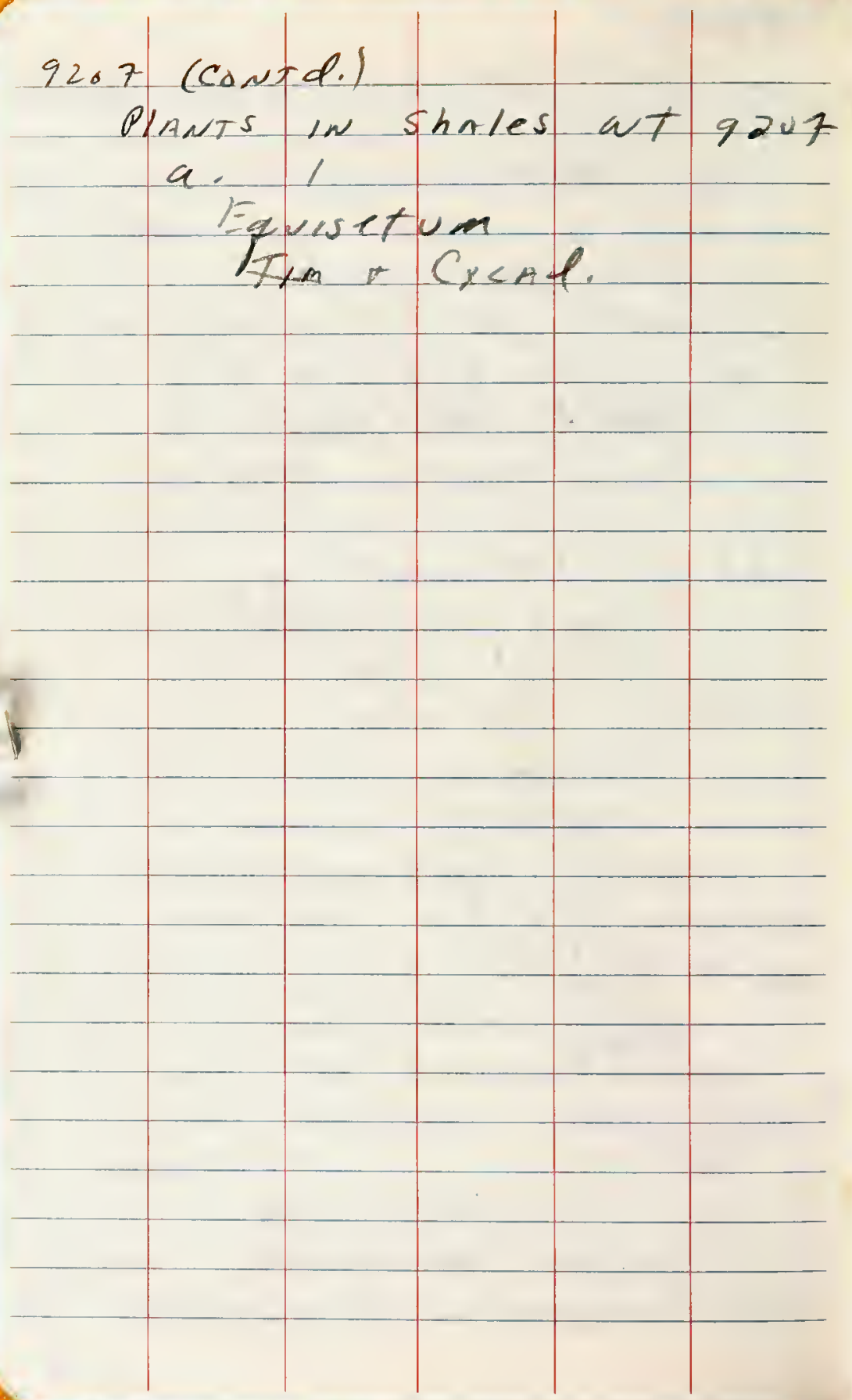




$$
5 / 22 / 92
$$

$9203 \mathrm{~b}$ CAytar A BCl again -

9203 C FurThest S. IN caytur bay at yous WAb

Clado

Milsar,a

Taenupteraj

Sphenubarera

Eeinitzia 


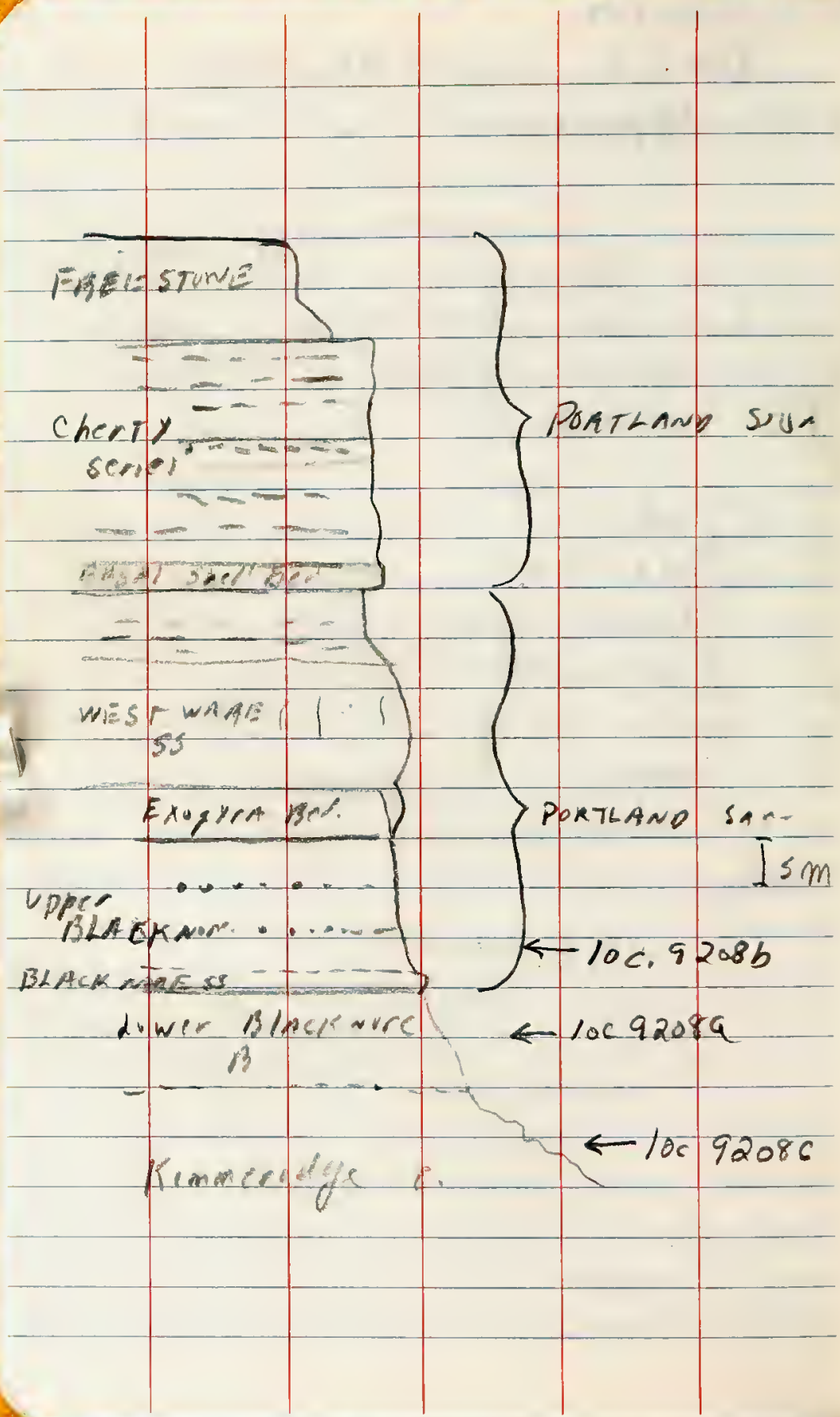


Dorset

cimber Beds

$60 \mathrm{~m}$ Lu/warth Bels

26.7 DortzAnd stome

Part lamblan

36.8 Portiand saivl

Krmmeradyian

- 65 Cor a lliar

- 42 Upper difurd Clay

$-\quad \begin{aligned} & 8 \\ & -19\end{aligned}$

Oxforedian

- 3.2 kelliways Bukiday

s Upper connbras 


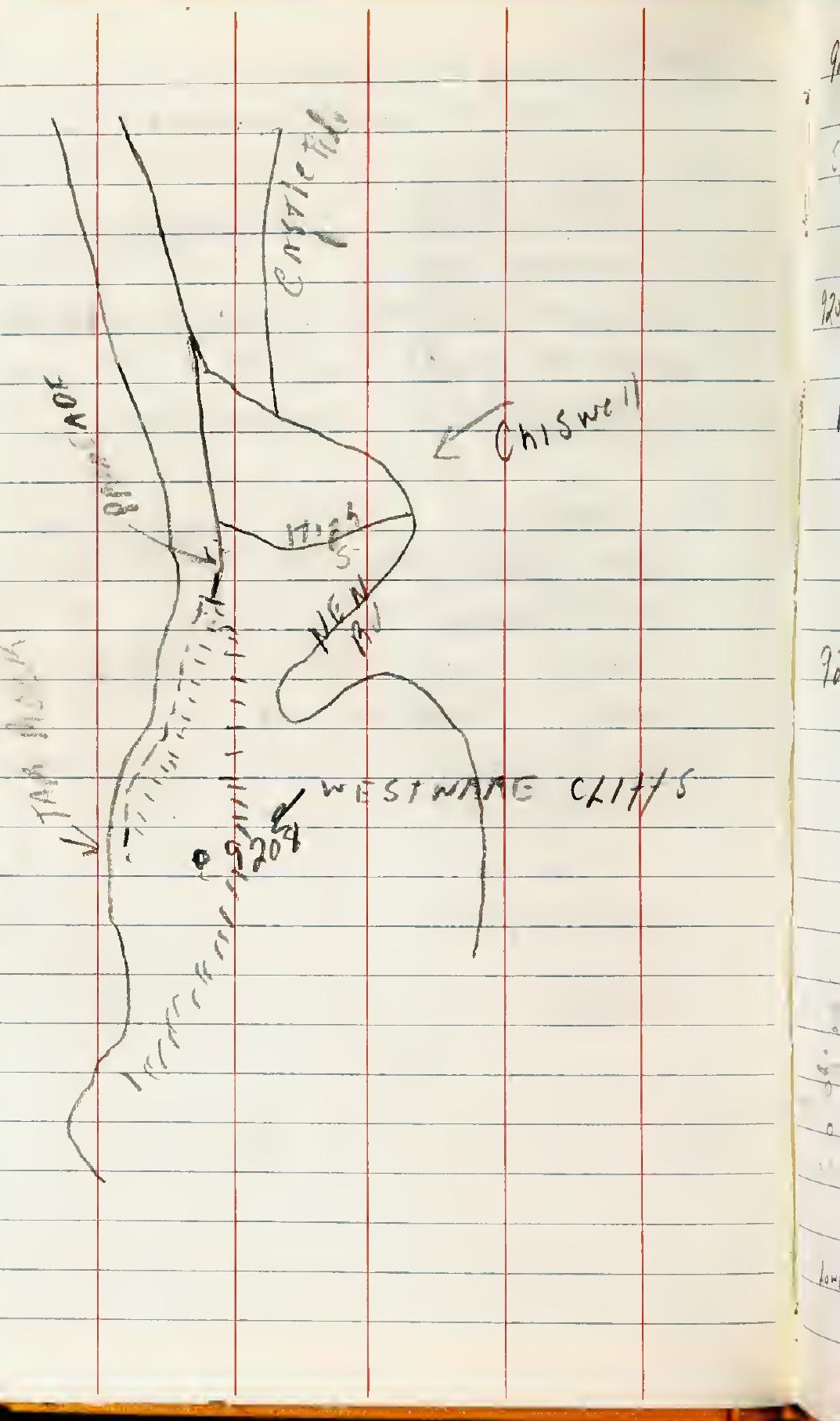


9208 West naRE ChiffS LOC.

$3124 / 95$ kac. I/A it GeJul Dorset COAST sy 68107248

9208 a Lower B/ack NoAE Mbr of ParTLAND SAND NeArshore marine.

2 SAmple! TAKAEN

9208 b upper BLACK NOME MSE it Dertiand sanel

w Clam shells

- Frum slump

$$
\nu^{\sin p}
$$

$$
\begin{aligned}
& x-92086
\end{aligned}
$$

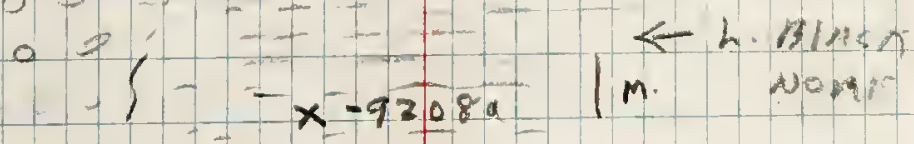

Lower BLACK Nore

a Grecwish Biack prob. 
ghucontic 5 :

$w$ pecten shells.

$9208 \mathrm{C}$ Kimmeridye ClAY

- Dark grep unctuous clay ar base of The TAM Basins Slump Block.

of The the of the slump just above berm of loose rock. 
9209 Furzy Clift toc. mip ref. $5 \times 69.981 .8$

Dorchester Sheet 194.

$0,33 \mathrm{~m}$ Below Red Nodule Bed of Bomlenze Clay wher of Upper Oxfore CINY Gryphrex frum snme towel $\rightarrow$ 72-09 opendwe somples 
9210 Enst ot Bowlense Cove mp nef. SY 70.781 .8

Nothe Clay of Coralliaw

ombelow the Bencliff

Gri?.

- SAmp 9210 for Olenvar

Eav. wear shore subtidaf. 
9211 peurs point

5124192

Fram Corbula beds of Durlster bels

-he uurlslon diels of Ehe Purke $A$ For.

At Type Amp of Murbecte on Durlstun Ras, w of SWAarge Seg comsists af do em to broken shell is w interheded Inmintied unetubus dark grev clays w shell hash.

$\rightarrow$ Sample g2/1 abour $300 \mathrm{~m}$ et tip af Peveil doprit. ir corbula oleawane Beds.

- Middle Purbeek in kowerimesi 15 
92116

Cinder bels sim above sea kevel

ON DurIston BAY

Pure Clay w Shells in - ir DArk grey carbon

- On. Durlsnin Bay $5 \mathrm{~m}$ whore S.L. 
9212 a swarage Bay $5 / 25192$ a Swirly North limb Swanazé B GS Sheet 343 (part 342)

Sheet 343 of Ged Survey $03,580,5$

Dorsetshire

WeAld CN Fm.

Carbonaceous SIT w

Comminuted plant matTER io med. grey, ferrujeneos stained kent if sig.

welled segihere consists of $x$ held ell ss tar cult a Flat y Trough x -bedded some sanguve

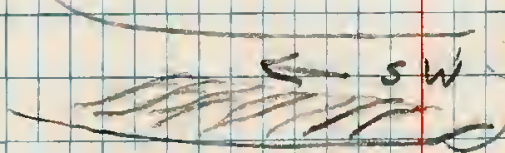

SS. InTernedeled at $4.5 \mathrm{~m}$. intervals we maroon to reddish mudstone w/ ped., thizdmorph 5745 indicating gley soils. 
This is a teresirlat seguence on a low flost plain (piob mende bejt w) a salsudal subtesplea Climato

$$
\text { B. }{ }^{2} p \times\left\{\begin{array}{l}
18-19 \\
20-21 \\
20<9212 a
\end{array}\right.
$$


Loc. 9212b sumange Bay at an access julley Juso s. of N.most grom

Sheer 343 0 $3,380.4$.

X dedted f to migriss

$w$ comminted p/ant MATTER

App $3 \mathrm{~m}$ expared in clift

Current dir.s.su.

Prombly 20 m heliw 9212a 
$92 / 3$ Studland B/AY $5 / 25792$ BGS Sheet 343 4.482.4

PX29-2r K/Eocene Chalk/ Rending be of uncunf. w fissure filling 
9214 StuOLANl.

Redend Sand.

Barshat Beds Focene

BGS Sheet $343 \quad 3,7582,8$

Black Phan Carbonnceous

ClAY in a red to

Bronin Ferrogincow

f.g.ss. w $x$-beds:

some mangmese striming

$\rightarrow$ Snmple 9214 . 


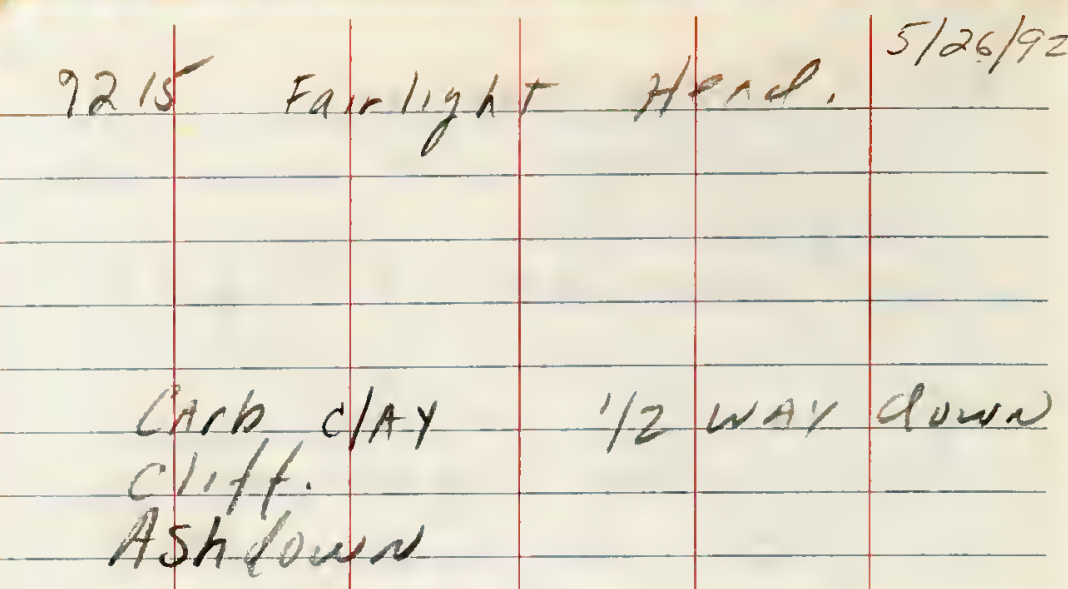
9215 b 9215 Besch Beldw

Trovgth chande) poINONA s. W Arits ot PAAN MATIEA W B.PP. plampi. 
$92 / 6$ Axis of Follight $5 / 24 / 92$

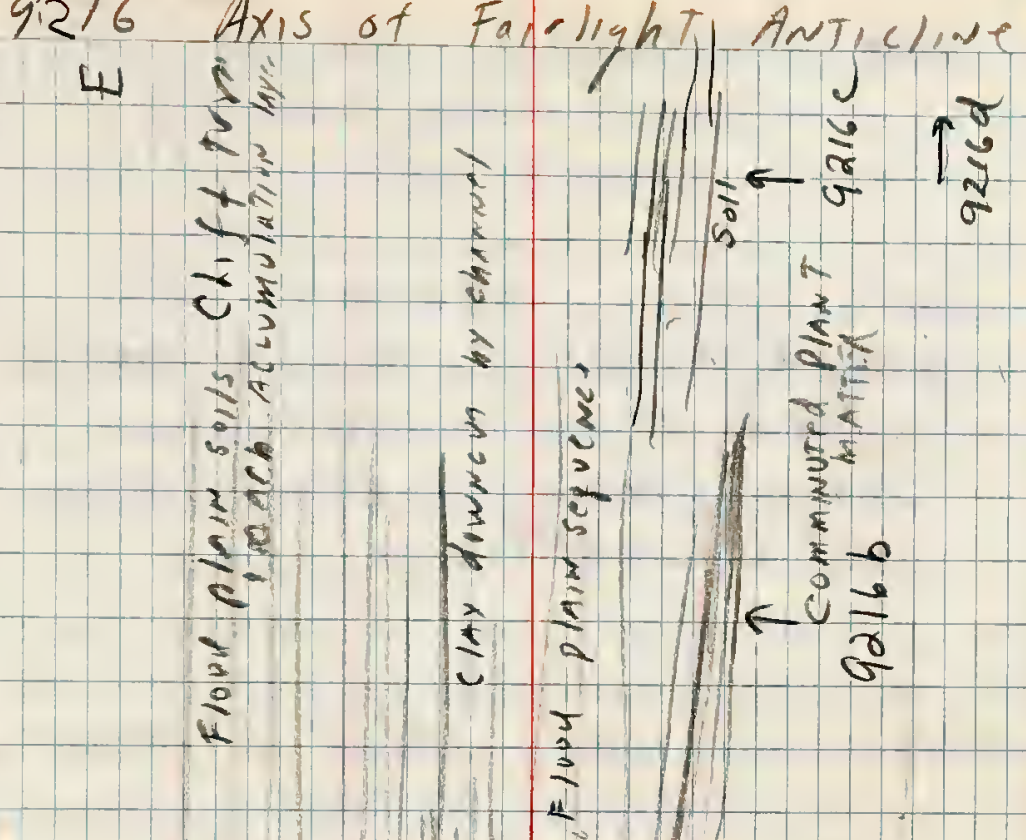

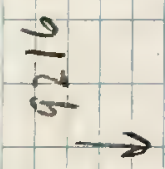

3
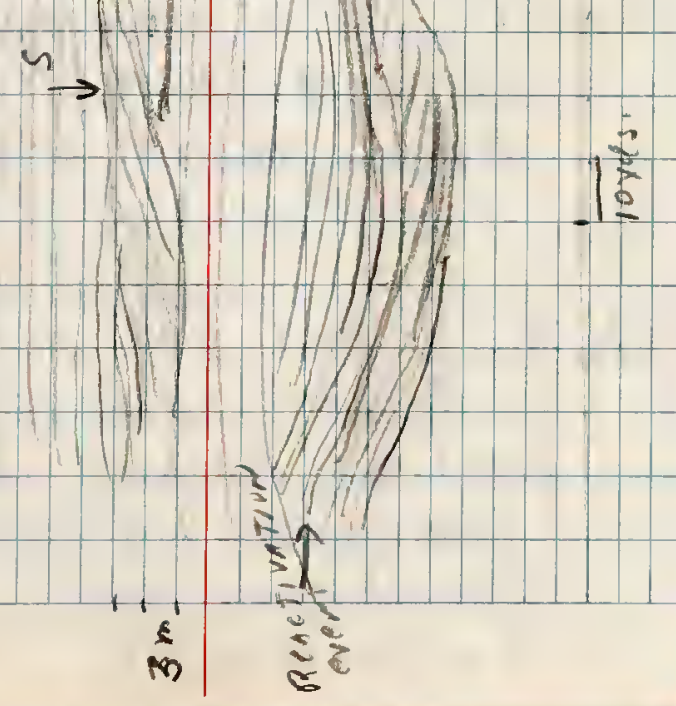


$$
216 \text { (cont) } \mid \text { maly } 26
$$

Looks like sequeate of meander belt Ss chanvels * flool basin solis. spme gleyry mort/y ad solized

9216 b cravasse splay deporLt or distal levee.

Ahout 100 yds e. Of view prorint 9216

9216 C. "B" N ACcumu/ATIun horizon ( Wumate) of floed plaw sequevere

9216d PHANT bed In weardewl DISTAI SPIAY dT SAmC revel as 9216 c Onycheopses feryle Brachrphrlum poncretum Ruffordial"? 
Sphenlepis

_PSeudocyCAS.

same level of distal splay UNIT 


$$
9217 \text { Burried enty J/26/92 }
$$

recint fupesi of

Tumps and sways

map ref:

Geol Survey /:150, ous

sheet 320/321

$1080 \quad 1282$

ptersi

Farly holicere

aा 5 , opo yes b.p. 
MAY 27,1992

9218 ockley Brtck BIT at

Smokejacks farm

Tq 11.237.5 Qu11.187

Donking.

Havterivian/Barrimind saly

Extensive parallel bedded carh \& Fernzheous WeAthe cing shale $20 \mathrm{~m}$ thikh w it grey silerite wudules about $12 \mathrm{~m}$ ahove hale of PIT:

Orie Tayoliod core Ockley mystery diant

- Beeties

Fish fromems

Abour $10 \mathrm{me}$ Heghen a BaryonyX (Walkeri: - and Iguapadon

$-0=5-2$

$3 \times 1$

$P \times, 4, D$ 

9219 . LAybrook BriLk works
upper Burreming

- Wealder G/ay

Ibstbck Bace pits

Tq 11618.9 sheet 182 .

on Brighton Sheet 198

wenid CVAY

paraliel bedutel

* Limmated w bloturbatives on sompe surfaces Anoxic Clay dan prey but This is rave as all motriel and showes some sigus yf at lerst some 0,574 rantipas.

worm tubes od harizowtal surfaces below romspme 
9220 WAOHUS ClAY of Weald Fm

In old lower pit of Freshlare Brick cu

Shoes laminated w SIIT layers w some slicks and vertical Equisetum

Paludal flood barm on lower delta plane.

Laminated nu broturhatrod Anodic (semi) w fresher
yworevals 
9221 New Dit of Freshlane Brick CO.

- Grinsted Clay a Upper Tinbridge hells sar li UTS is a series ox Shallu, fuulsion channels $3 \mathrm{~m}$ Thxik 4 apow $100-$ $200 \mathrm{~m}$ pronl. LATernl to these is a seyvewce of Typical Red Greentu hlack motrled colis bleds (AnTApsco) mpk m Ihe clays ron.earbumacefis r so wos Sample.

Fourd "peblete bed" at Top Di lowef Junbrilge wells

NoT suirahle for sampling 
$9222-9230 \mathrm{used}$

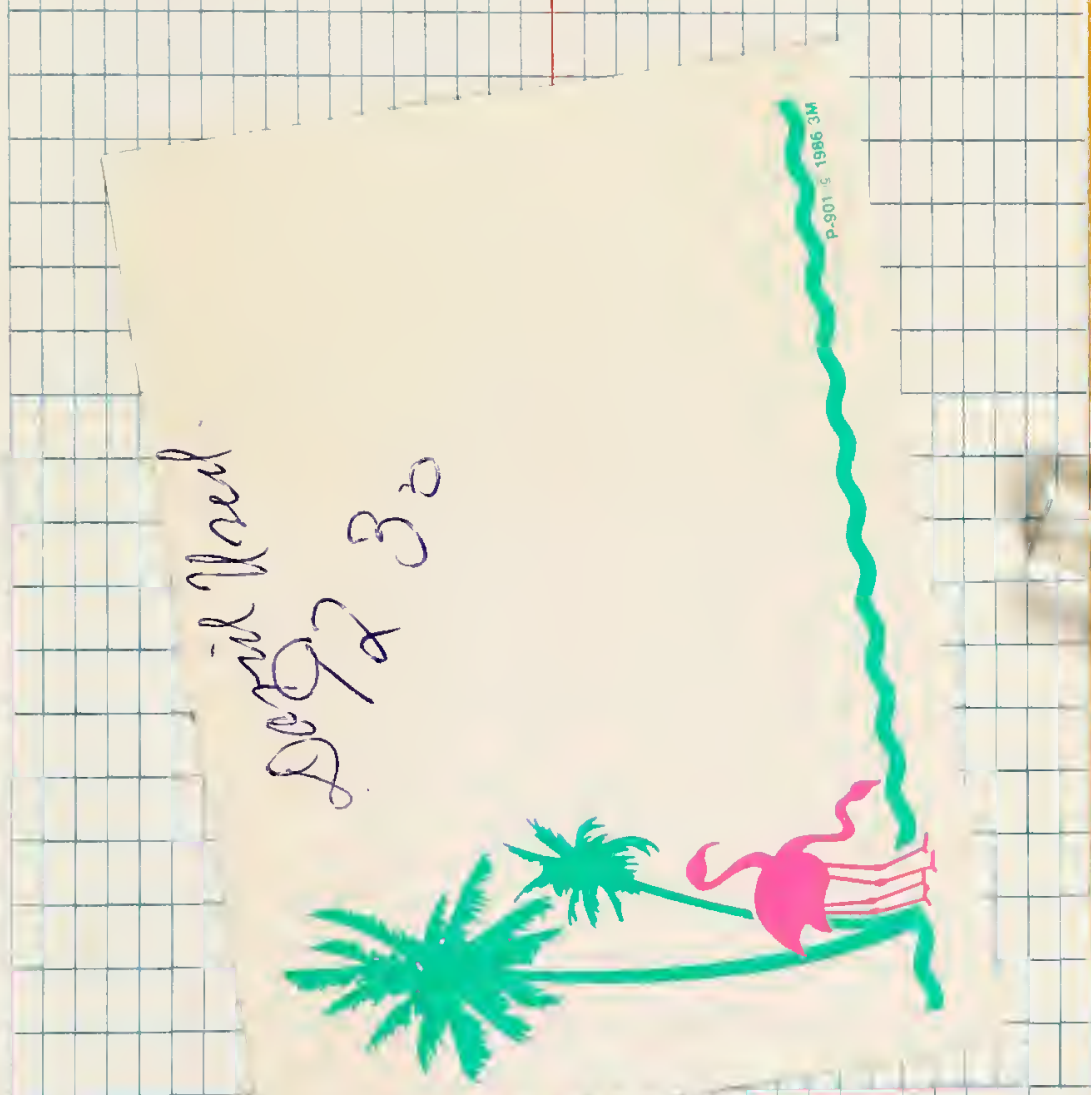




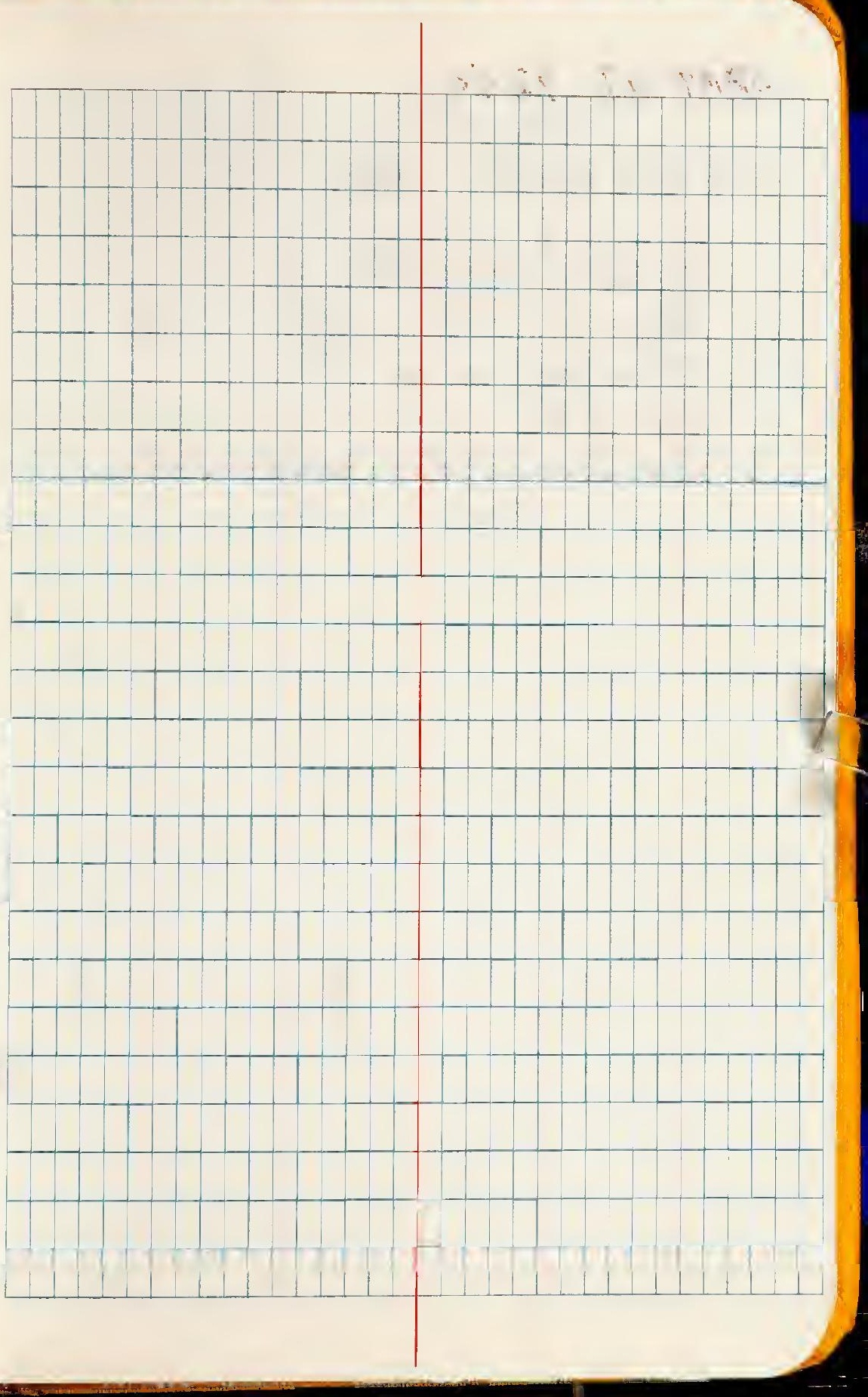


SAat at 9240

6119

BCh site 23.

Washimatomites

Araventites long. Co 7

Fruiting axis

INO 10

DN 20 BCR SERAmbler 
SUNNY, hOT, S STIII.

AT BCR South end:

$6 / 25$

Plotice position of

SITES 42-47 on

Aerial Photograph.

122.3 from nut hole to
Mat hab 
Lot. $38 A$

$6 / 26 / 92$

Large Prim leaf of washing powites $15 \mathrm{~cm}$ above soil Ayer px by scots kat is Twisted counter clocknows to or left. side especially af base 
$7 / 3 / 92$

meeteetre Dump w

Kay Behreas melyer

Brian

Scust

gubla Murnham.

Soils noi lenkhed. snovid peture for extra collecring 
9241

$$
7 / 3 / 22
$$

Revisined loc $1133 \times 1 / 34$ 
$B C R$

7/6/92

JUST $201 \mathrm{~N}$ of site 36

Substrate of soil relapsed

SANdY ClAY Almost no soil develop-

Plants Acer Cred very imps. 


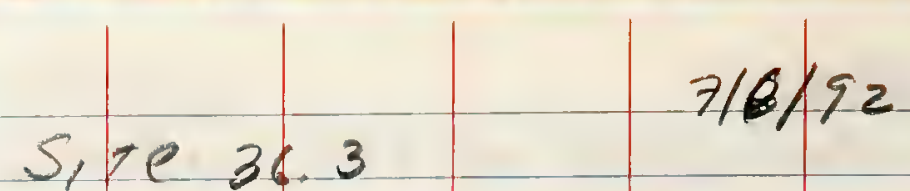

VRM $20 \mathrm{~cm}$ across $w$ shenthing menvly circular lenves that fill w/ selimen. 


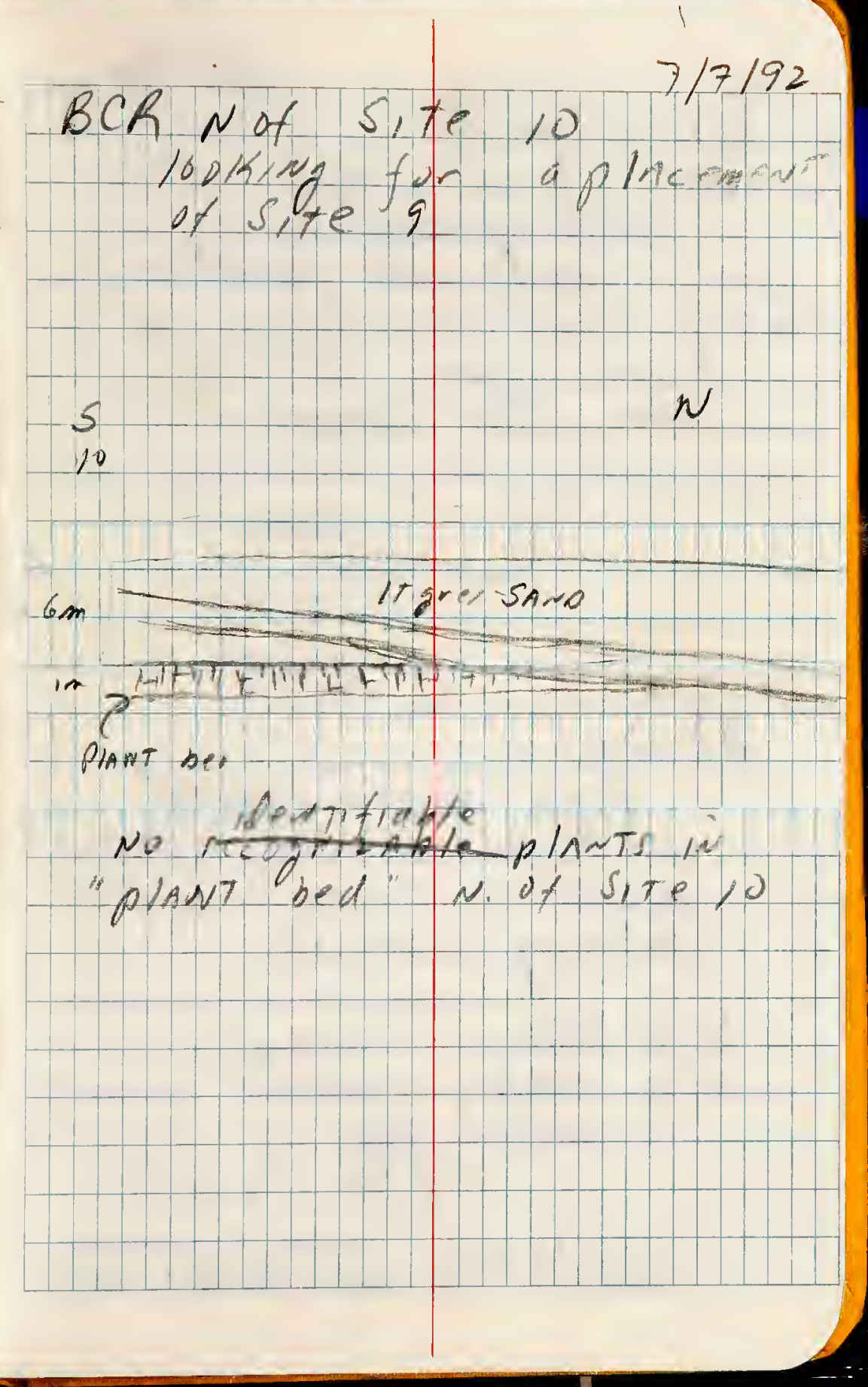




$\mid$\begin{tabular}{l|l} 
Discussion & $7 / 8 / 92$
\end{tabular}

Stindiry water ouly in powl at 50 -

Demsly pranale sail w elay lamente

A Senvall

High WAtir Titile wot flooded but salden fiess.

Bedueruy subtrait

Dies Irac

Tuff fiwiwg upwids alwros in furtab cm figss to 517 .

PIANT HASA at 10-24 cm 
level.

Pint hash possibly

mostly water lN ow

* from they up to top

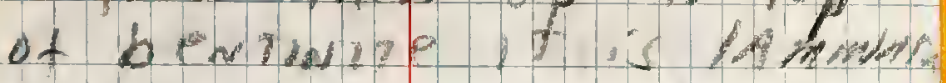

Press boned still water IAminatide

- One eruption

- (Air)

- Pontine

- Burial of remain plants.

- Arr fall for origan 


$$
\text { SITe } 18 \text { Redialing| }
$$

This was right down to the soil horizon and below The pressbated 
$7 / 10 / 9=$

$B C B$

AT Site 18.1

Consusing N

No pressbond at Thas

SITE

Also almost no Anemin frementil Aluhowe this is fominum iT phT is $8 \mathrm{~m}$ to The north. 

Site 18.3

$$
7 / 14 / 9=
$$

- Sorlhere is bedded with beds of melt p w/ cart SilT.

layers of unrewarded plant incl.

Palm fronts $y$.

Aravearites found here Much fustic in soil. tuft is sharply based $w$ only series of plant material incl. palms y leader fern frags very rarely found.

Some convoluted rs noted in Tuff

SITE AnANdund for PIANT Census. 


$$
7 / 4 / 92
$$

Site 18.4

SITE Abnedonel fer lack of fessits.

Inotes recorded ow data capture sheers gever to A.te Behreasmever. 
$7115 / 92$

Meeteetse Section

3 Bentursites ar bare of se-kon

istuss above PMAG MTSAI

2 2nd. 2 Im helow MTE 42

3rd Just below MTiE 44

Door Thini DEy MTE 45

Ahore MTE 16 pour

2 In LANCE AT ROAd intersecsin Do FArm

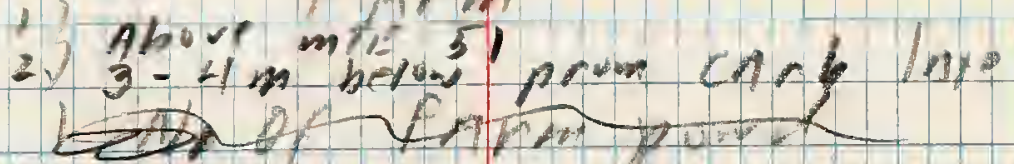
beiween mis 52-53

FArm porl.

below Mre 54 


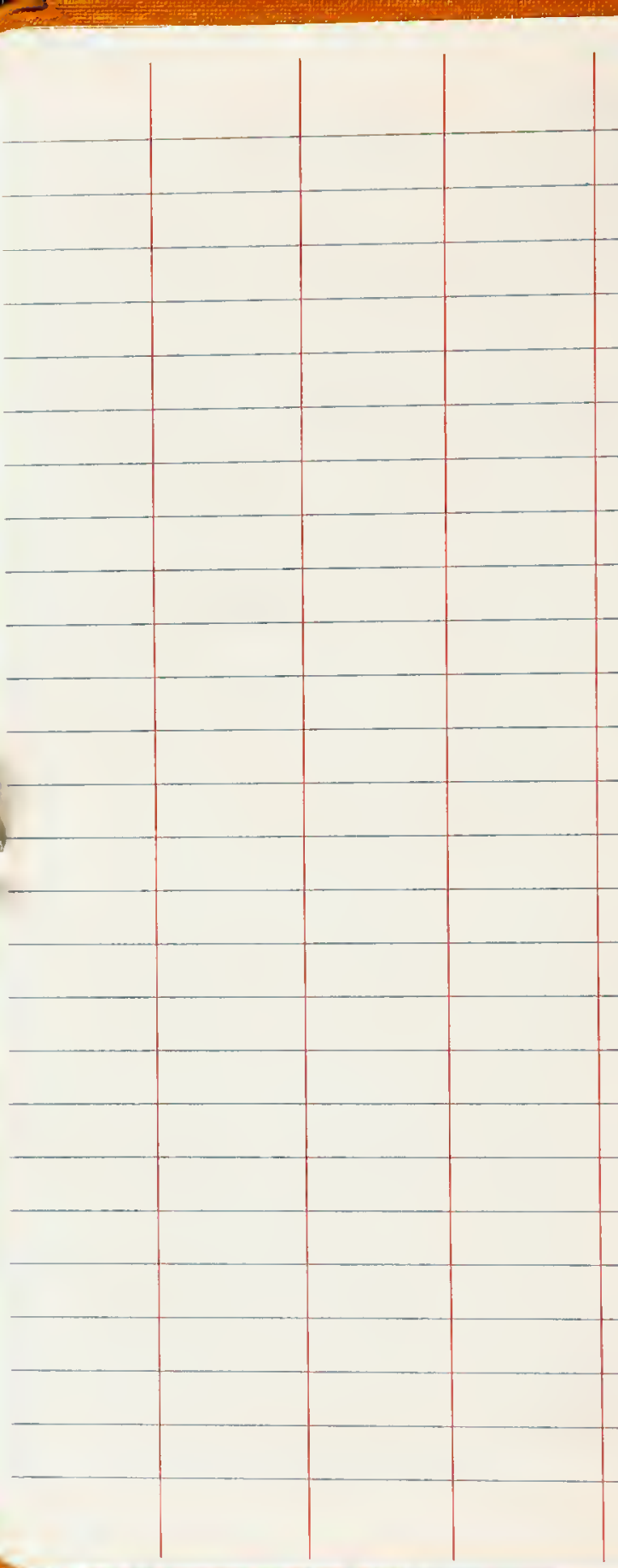




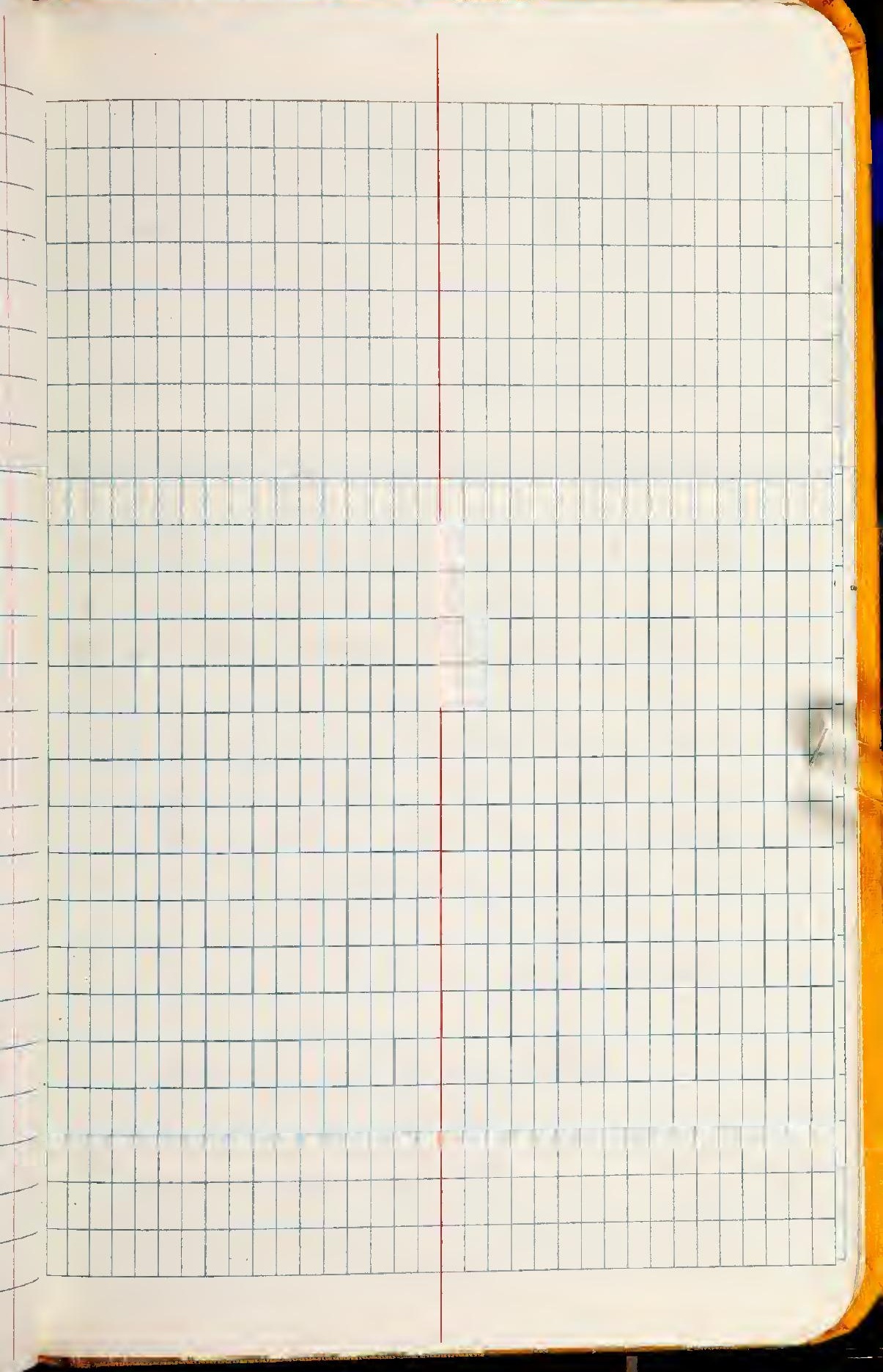


$7 / 18 / 92$

$9242=M O-1$

Eurvey of mainfovil plat 50 e on provarde wose in meetedre dirall

Cewsus completed at 4:50 p.m. as thurderstarm apprapered

Thu cretacea / SLW unezwhelmingly. ant more than 998

MANY STIUK

at chuces wood masses of thujits of cur fossil phants

Area of pit

$1.5 \times 2.5 \times 3 \mathrm{~m}$

2.5 


$$
7119 / 92
$$

RAin day up field wonk. 


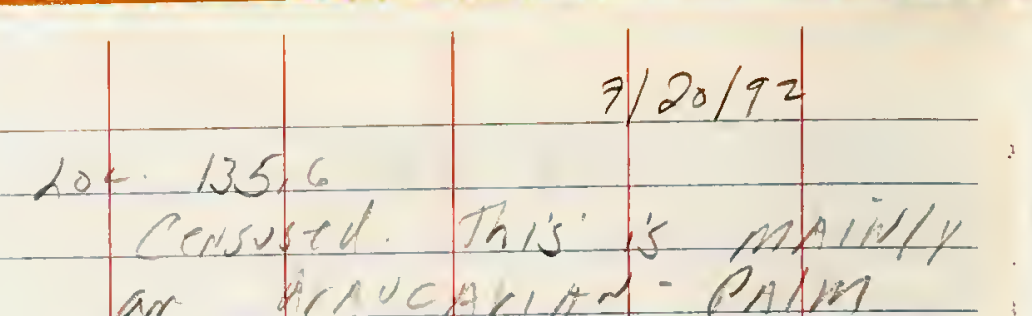
ar Pencherity-PAXm drminhted ss app a

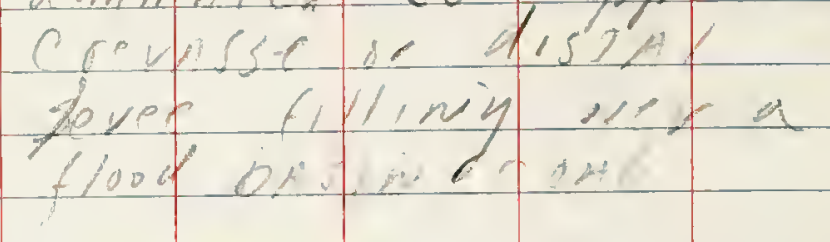
Swht seetid ow consus
spet 


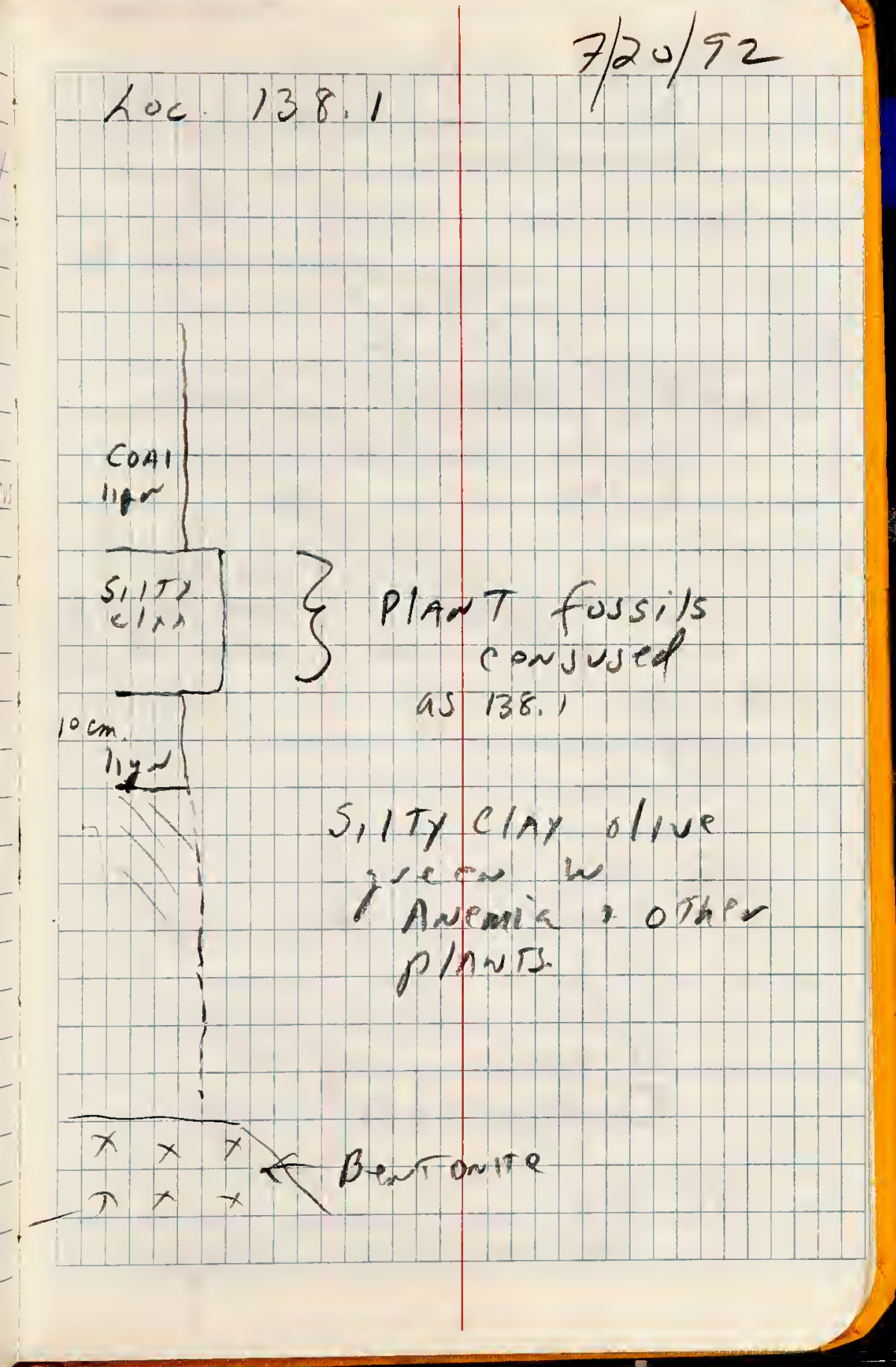




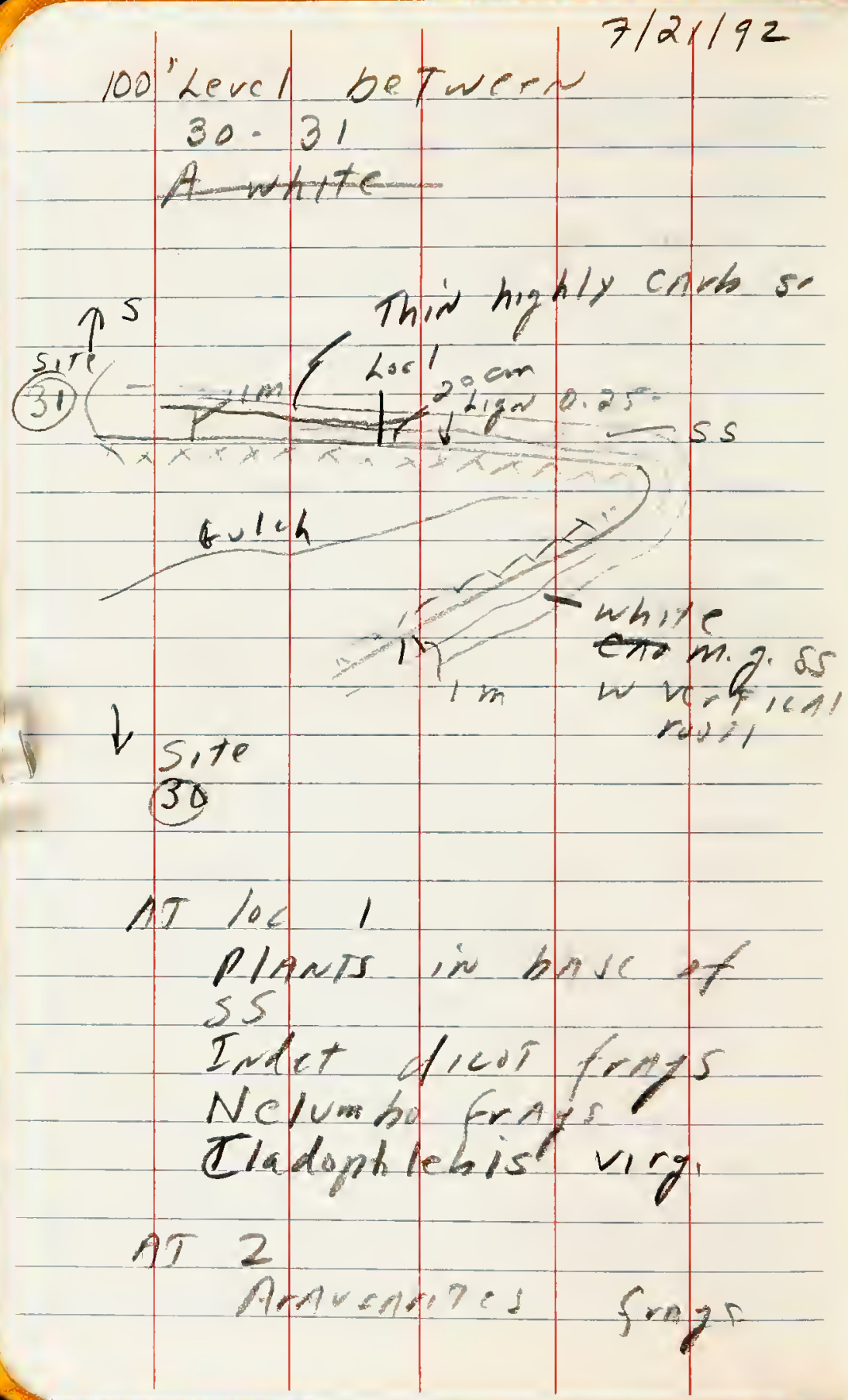


$7122 / 92$

AT SITE 242

PIT Size $2 \times 3 \mathrm{~m}$

Fossils sparse

Sec. observations made on standard sheet. 


$$
\begin{array}{l|l|l|l|l|l|}
5179 & \text { a. BCh } \\
16.4 & 7123192
\end{array}
$$

PaIm letef found it $12 \mathrm{~cm}$ above soil Appiny doun to $8 \mathrm{~cm}$ on alstal portion Also farol we Avemia fremente and frogments

Press bond layes at about $25 \mathrm{~cm}$ nave soil laper 
Site $14.4 \quad 7 / 23 / 9=$

- Pressbanel nt as cm above bare soil

Found mar sell $40 \mathrm{~cm}$ above sail.

No fossils to pAse of bestial. except for palms right at base. 
$7125 / 92$ site $(-716$

Clinker direlopled foos prominent $2 \mathrm{~m}$ ligaite * ligutin shlite

PIANTS found

Eodic ranopteris

Indet monacot

203

Indet dicot if r $12 \mathrm{~cm} 10 \mathrm{mp}$

Ancmia fremitritis

Verticil rooted moro 

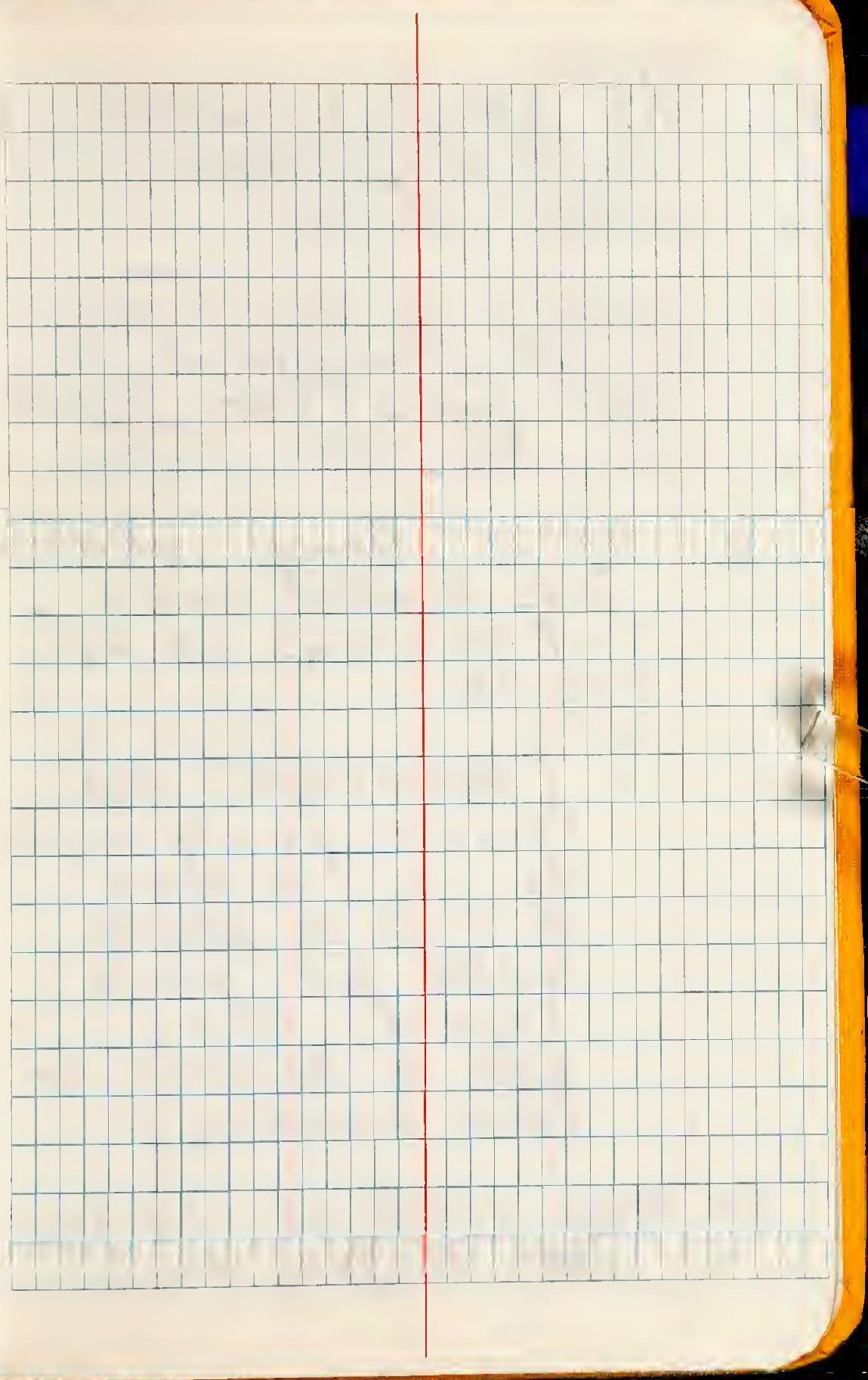
$7 / 29 / 92$ AT Base of Jason midge sfortion

STAnTon on the highest kedge ferrugincols ss That forms the top of
the fox Hills Fin

O Bp at Brow impure flat bedded conHills $\mathrm{Fm}$ UNIT

0 Mecteetse Fin Friable dourly export

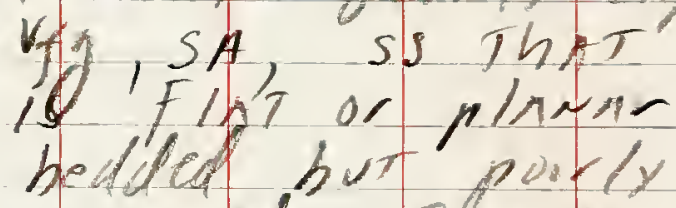
belled but poorly reposed fores hale of necteetse in [wave Donematch]

$2 \mathrm{~m}$ Base STAKE Of JH Sect. same IT. grey SS- 


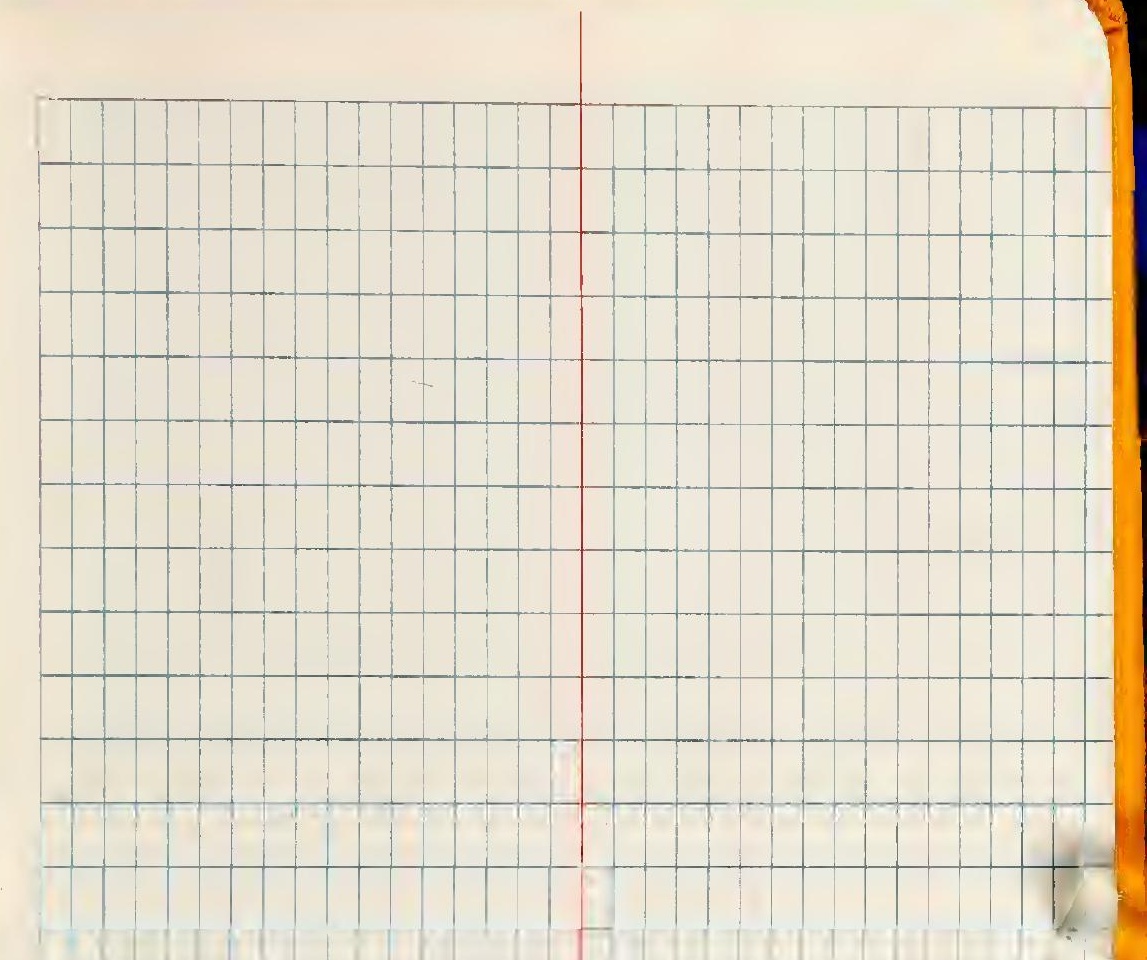

$+$ 


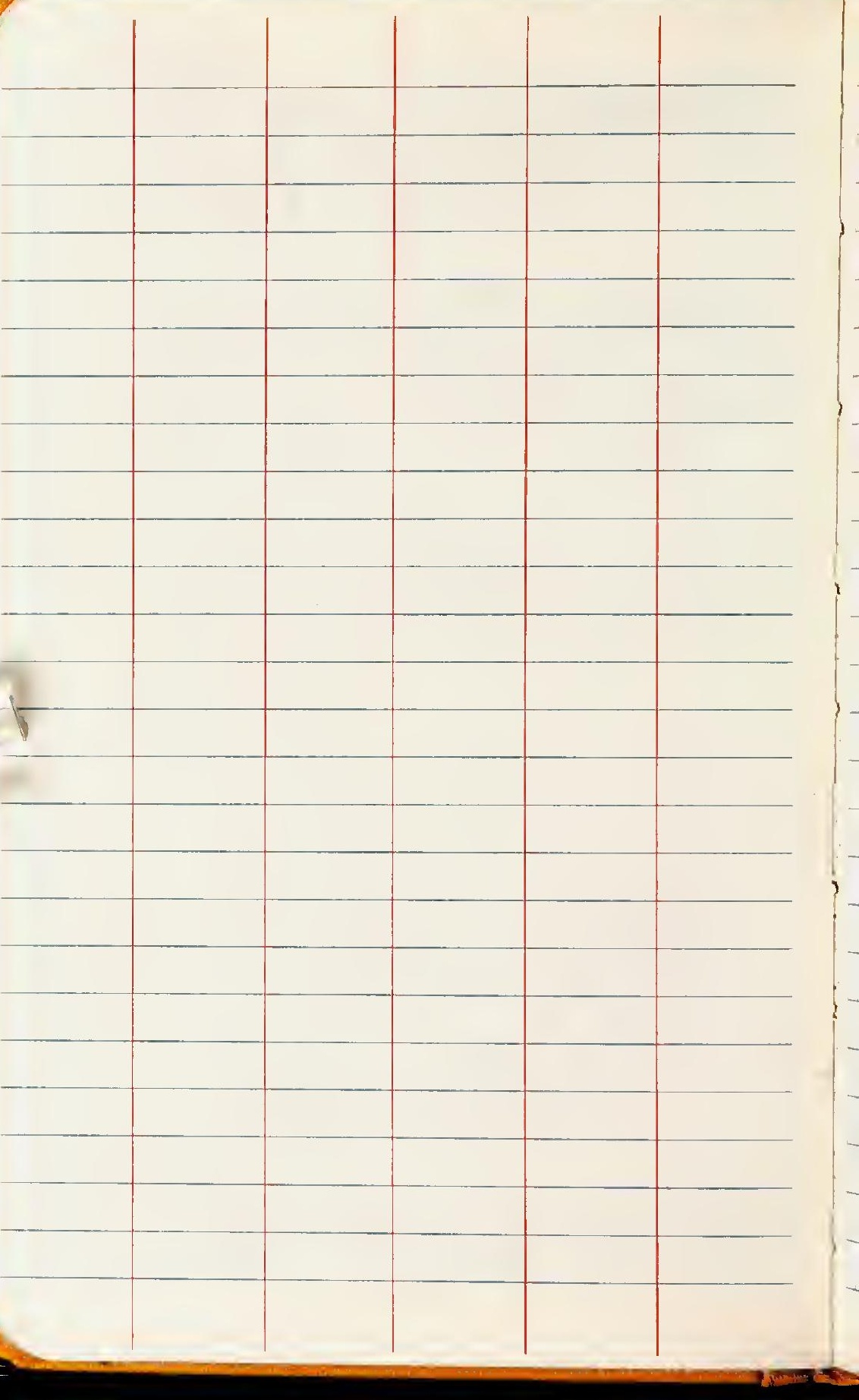




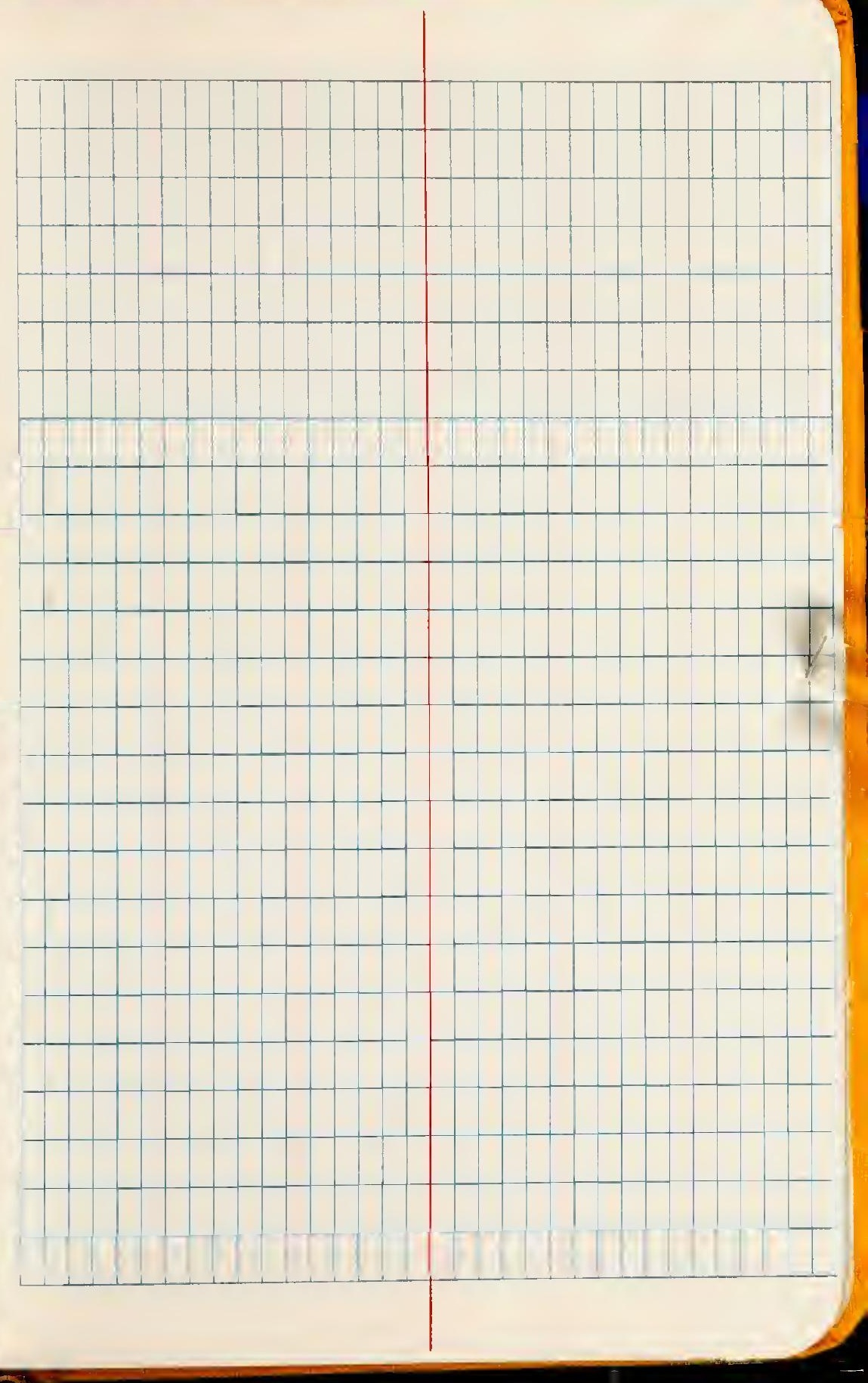



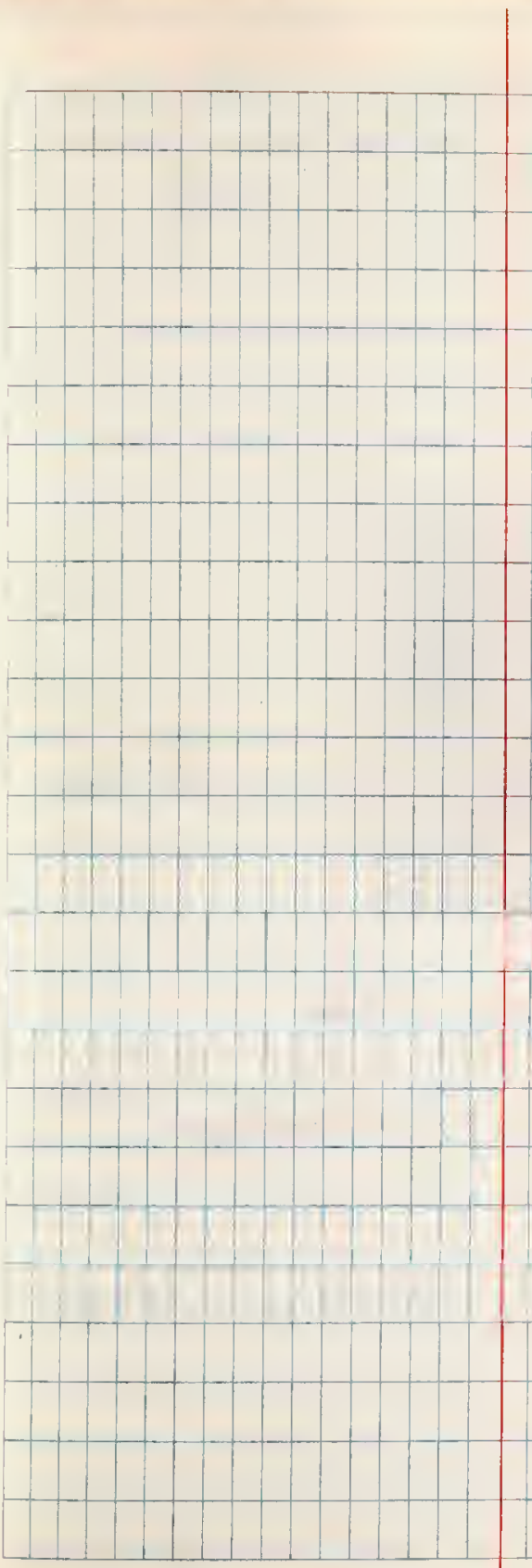


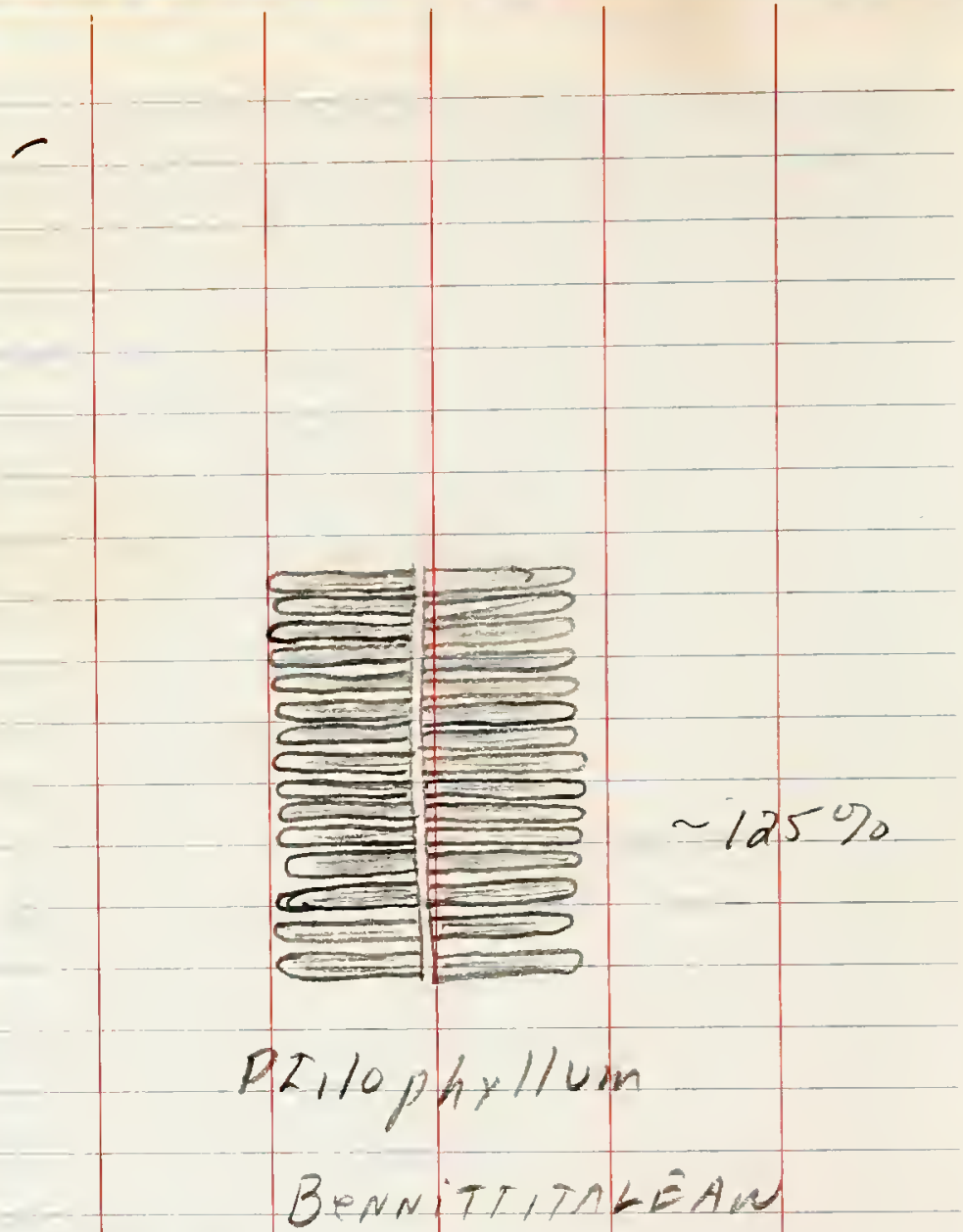



$\mid$

Stum Stexpoing

Put

Axibul toper the

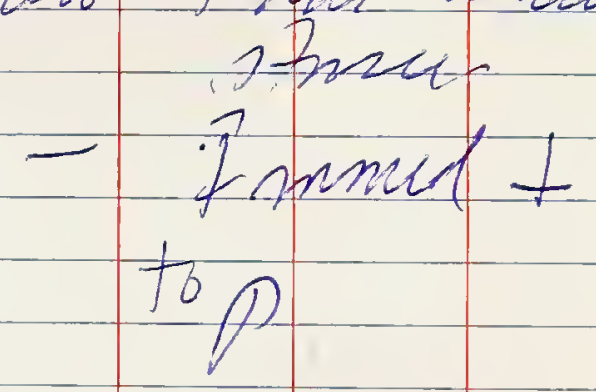

Fruthol \& - Dareted

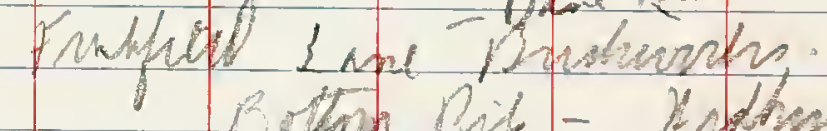

Botan Pite - Xendanz

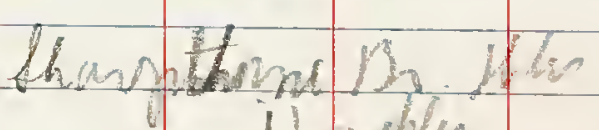

Howlen 


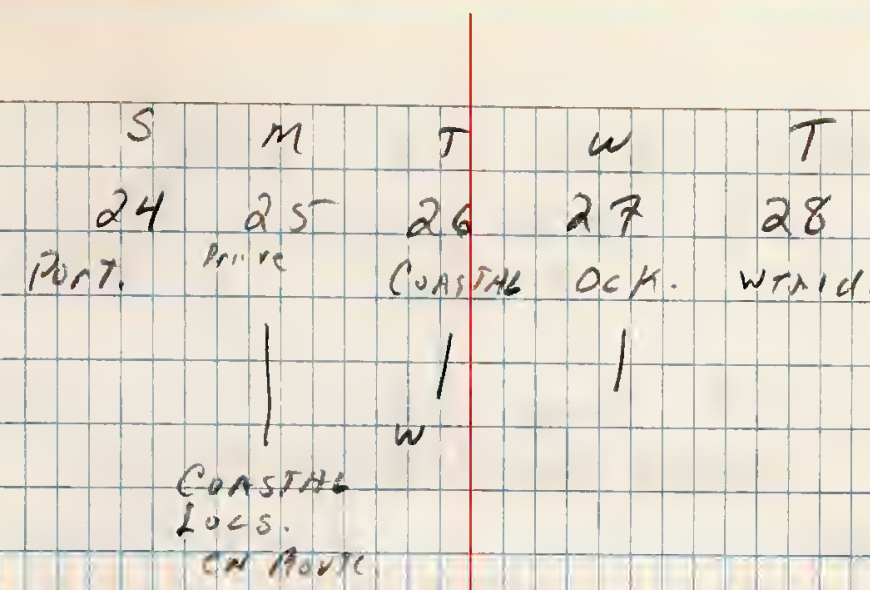


Meet Chris at 9:30 ait Snoke jackis rarenc= wonks. in Ockley

0.5. Map. 187 (1:5i, 000 Sa) Grid kef to 116372 Chis - mus. 0719389483 - mus $\begin{array}{r}0719389423 \\ \text { dis } 081940.6643\end{array}$

Jasor Hicks

to Acknowledge Cherrin in his phpers

Noton Wilh - Hastines oul 7 0424428168

Pin Heths

$$
0798.872348
$$




\section{INDEX OF \\ CURVE AND REDUCTION TABLES}

Table I-SLOPE STAKE

Table II-STADLA CORRECTION AND HORIZONTAL DISTANCES

Table III-TRIGONOMETRIC FORMULAE

Table IV-NATURAL TRIGONOMETRICAL FUNCTIONS

CURVE FORMULAE

Table V-TANGENTS AND EXTERNALS TO A $1^{\circ}$ CURVE USEFUL RELATIONS

Table VI-INCHES TO DECIMALS OF A FOOT

Table VII-MINUTES IN DECIMALS OF A DEGREE

Table VIII-MIDDLE ORDINATES OF RAILS

Table IX-SHORT RADIUS CURVES

Table $\mathrm{X}$-RODS IN FEET, 10THS AND 100THS OF FEET

Table XI-LINKS IN FEET, 10THS AND 100THS OF FEET

$$
\begin{aligned}
& \text { left blar } \\
& \text { lett Haw IL } \\
& \text { lefer }
\end{aligned}
$$


TABLE I. SLOPE STAKE

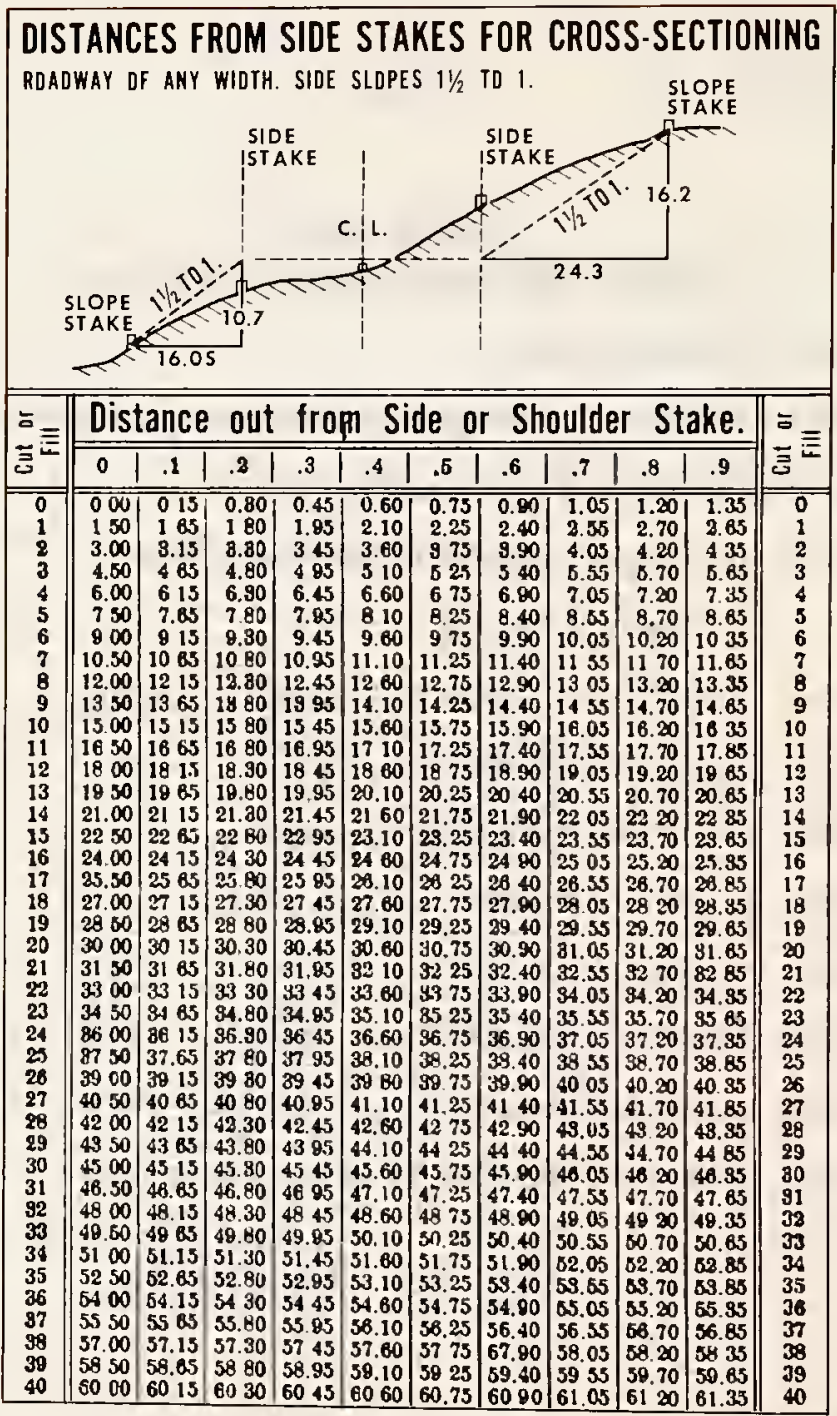


TABLE II. STADIA CORRECION AND HORIZONTAL DISTANCES

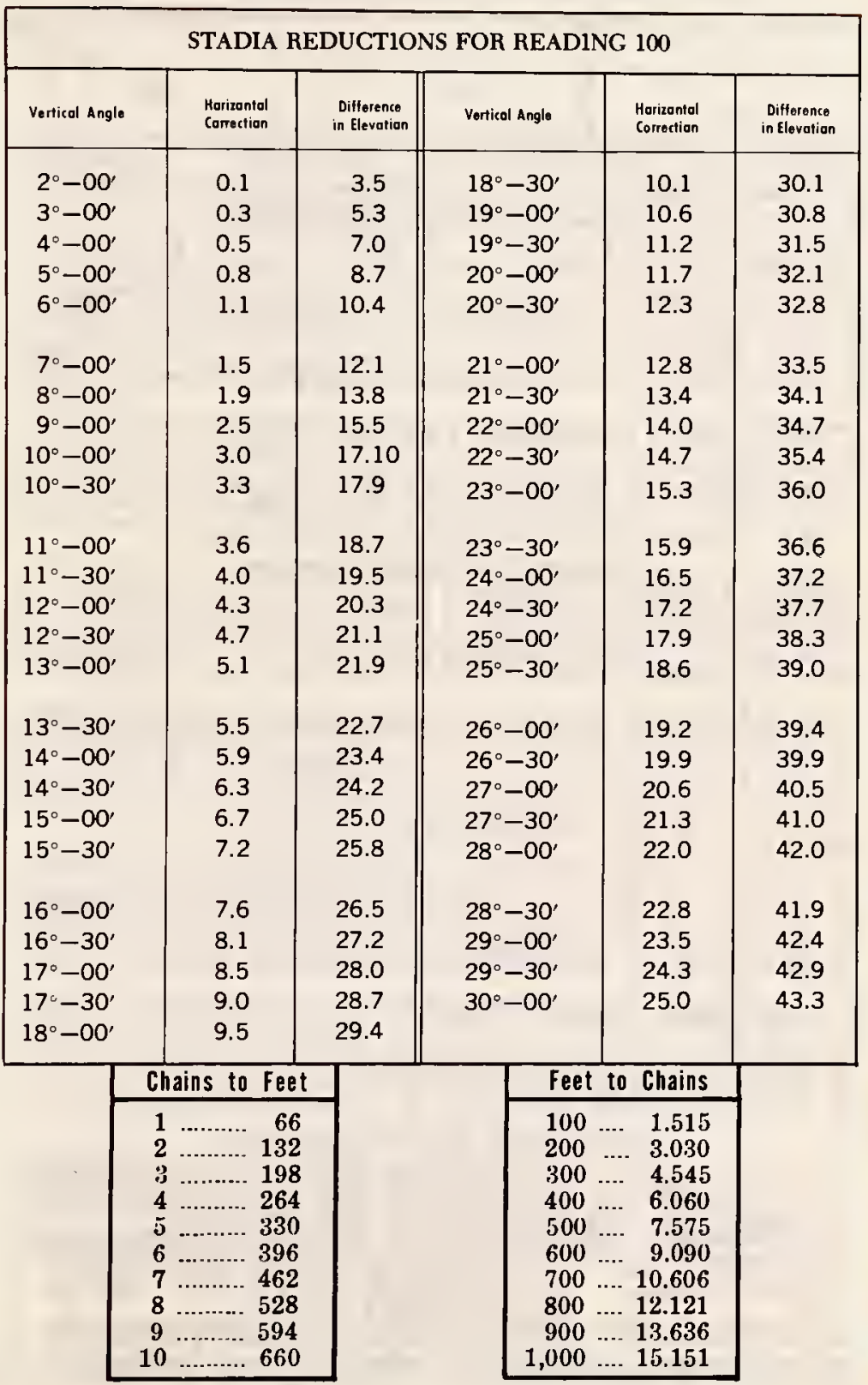




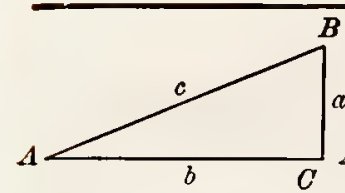

Right Triangle

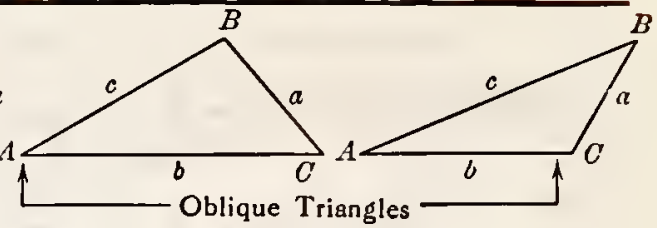

Solution of Right Triangles

For Angle $A, \sin =\frac{a}{c}, \cos =\frac{b}{c}, \tan =\frac{a}{b}, \cot =\frac{b}{a}, \sec =\frac{c}{b}, \operatorname{cosec}=\frac{c}{a}$

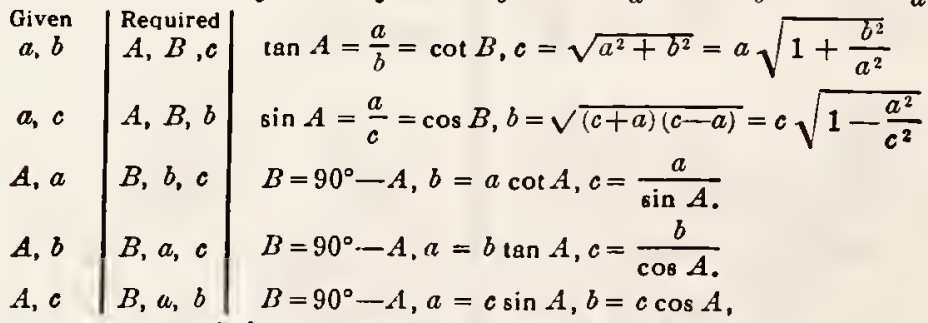

Solution of Oblique Triangles

$A, B, a\left|\begin{array}{l}\text { Gequired } \\ b, c, C\end{array}\right| \quad b=\frac{a \sin B}{\sin A}, C=180^{\circ}-(A+B), c=\frac{a \sin C}{\sin A}$

$A, a, b \quad B, c, C \quad \sin B=\frac{b \sin A}{a}, C=180^{\circ}-(A+B), c=\frac{a \sin C}{\sin A}$

$a, b, C \quad A, B, c \quad A+B=180^{\circ}-C, \tan \frac{1}{2}(A-B)=\frac{(a-b) \tan \frac{1}{2}(A-B)}{a+b}$.

$$
c=\frac{a \sin C}{\sin A}
$$

$a, b, c$

$A, B, C$

$s=\frac{a+b+c}{2}, \sin \frac{1}{2} A=\sqrt{\frac{(s-b)(*-c)}{b c}}$,

$$
\sin \frac{1}{2} B=\sqrt{\frac{(x-a)(*-c)}{a c}}, C=180^{\circ}-(A+B)
$$

$s=\frac{a+b+c}{2}$, area $=\sqrt{s(s-a)(s-b)(s-c)}$

$a, c$ Area

$A, b, c$ Area

area $=\frac{b c \sin A}{2}$

$A, B, C, a \mid$ Area $\quad$ aré $=\frac{a^{2} \sin B \sin C}{2 \sin A}$

\section{REDUCTION TO HORIZONTAL}

Horizontal distance = Slope distance maltiplied by the cosine of the vertical ankle. Thus: slope distance $=319.4 \mathrm{ft}$.

Vert. anple $-5^{\circ} 10^{\prime}$. From Table. IV. $\cos 5^{\circ} 10^{\circ}=9959$.

Horizontal distance $-319 .+9454=318.09$ ft.

slope distance

ב Horizontal distance alsc-Slone dislance minus slope distance umes 11 -cosine of vertical anule). Wuth the same fixures as in the precedinf example. the follow:

Horizontal distance ing result is obtained. Cosine $5^{\circ} 10^{\circ}=.9959 .1-.9259=.0041$.

When the rise is known. the horizontal distance is approximately:-the slope distance less the square of the rise divided by $t$ wice the slope distance. Thus: rise $=1+1 \mathrm{ft}$. slope distance $=302.6 \mathrm{ft}$. Horizontal distance $=302.6-\frac{14 \mathrm{~V}}{2 \times 302.6}=302.6-0.32=30228 \mathrm{ft}$. 
TABI.E IV. NATURAL TRIGONOMETRICAL FUNCTIONS

\begin{tabular}{|c|c|c|c|c|c|c|c|c|c|c|c|c|c|c|c|}
\hline Angle & $\operatorname{Sin}$ & Ton. & Ses. & coser. & Cotg. & Cosin. & & Angle & Sin. & Ton. & Soc. & Cosec. & Cotg. & Cosin. & \\
\hline - & & & & & & & & & & & & & & & \\
\hline 0 & $\mid \begin{array}{c}0 \\
0029\end{array}$ & 0 & 1. & $\infty$ & $\infty$ & 1. & 90 & 8 & .1392 & .1405 & 1.0098 & 7.185 & 7.115 & .99027 & 82 \\
\hline 10 & $\begin{array}{l}.029 \\
.058\end{array}$ & .0029 & & 343.8 & 343.8 & 1. & 50 & 10 & .1421 & .1435 & 1.0102 & 7.040 & 0.968 & .98986 & 50 \\
\hline 20 & .0058 & .0058 & & 171.9 & $171^{\circ}$ & .99998 & 40 & 20 & .1449 & .1465 & 1.0107 & 0.900 & 6.827 & .98944 & 40 \\
\hline 30 & .0087 & .0087 & & $\mid 114.6$ & 114.6 & .99996 & 30 & 30 & .1478 & .1495 & 1.0111 & 0.760 & 0.691 & .98902 & 30 \\
\hline 40 & .0116 & .0116 & 1.0001 & 85.94 & 85.94 & .99993 & 20 & 40 & .1507 & .1524 & 1.0115 & 0.636 & 0.501 & .98858 & 20 \\
\hline 50 & .0145 & .0145 & 1.0001 & 6870 & 68.75 & .99989 & 10 & 50 & .1536 & .1554 & 1.0120 & 0512 & 0.435 & .98814 & 10 \\
\hline 1 & .0175 & .0175 & 1.0002 & 57.30 & 57.29 & .99985 & 89 & 9 & .1564 & .1584 & 1.0125 & 6.394 & 6.314 & .98760 & 81 \\
\hline 10 & .0204 & .0204 & 1.0002 & 49.11 & 49.10 & .99979 & 50 & 10 & .1593 & .1614 & 1.0129 & 0.277 & $6.197 \mid$ & .98723 & 50 \\
\hline 20 & .0233 & .0233 & 1.0003 & 42.98 & 42.96 & .99973 & 40 & 20 & .1622 & .1644 & 1.0134 & 0.166 & 6.084 & .98676 & 40 \\
\hline 30 & .0262 & .0262 & 1.0003 & 38.20 & 38.19 & .99900 & 30 & 30 & .1650 & .1673 & 1.0139 & 6.059 & 5.976 & .98629 & 30 \\
\hline 40 & .0291 & .0291 & 1.0004 & 34.38 & 3437 & .99958 & 20 & 40 & .1679 & .1703 & 1.0144 & 5.955 & 5.871 & .98580 & 20 \\
\hline so & .0320 & .0320 & 1.0005 & 31.26 & 31.24 & .99949 & 10 & 50 & .1708 & .1733 & 1.0149 & 5.855 & 5.769 & 98531 & 10 \\
\hline 2 & .0349 & .0349 & 1.0006 & 28.65 & 28.64 & .99939 & 88 & 10 & .1736 & .1763 & 1.0154 & 5.759 & 5.671 & .98481 & 80 \\
\hline 10 & .0378 & .0378 & 1.0007 & 2645 & 26.43 & .99929 & 50 & 10 & .1765 & .1793 & 1.0160 & 5.665 & 5.576 & .98430 & 50 \\
\hline 20 & .0407 & .0407 & 1.0008 & 24.56 & 24.54 & .99917 & 40 & 20 & .1794 & .1823 & 1.0165 & 5.575 & 5.485 & .98378 & 40 \\
\hline 30 & .0436 & .0437 & 1.0010 & 22.93 & 22.90 & .99905 & 36 & 30 & .1822 & .1853 & 1.0170 & 5.488 & 5.396 & .98325 & 30 \\
\hline 40 & .0465 & .0466 & 1.0011 & 21.49 & 21.47 & .99892 & 20 & 40 & .1851 & .1883 & 1.0176 & 5.403 & 5.309 & .98272 & 20 \\
\hline so & .0494 & 0495 & 1.0012 & 20.23 & 20.21 & .99878 & 10 & 50 & .1880 & .1914 & 1.0181 & 5.320 & 5.226 & .98218 & 10 \\
\hline 3 & .0523 & .0524 & 1.0014 & 19.11 & 19.08 & .99863 & 87 & 11 & .1908 & .1944 & 1.0187 & 5.241 & 5.145 & .98163 & 79 \\
\hline 10 & .0552 & .0553 & 1.0015 & 18.10 & 18.07 & .99847 & 50 & 10 & .1937 & .1974 & 1.0193 & 5.164 & 5.066 & .98107 & so \\
\hline 20 & .0581 & .0582 & 1.0017 & 17.20 & 17.17 & .99831 & 40 & 20 & .1965 & .2004 & 1.0199 & 5.089 & 4.989 & .98050 & 40 \\
\hline 30 & .0810 & .0612 & 1.0019 & 16.38 & 16.35 & .99813 & 30 & 30 & .1994 & .2035 & 1.0205 & 5.016 & 4.915 & .97992 & 30 \\
\hline 40 & .0640 & .0641 & 1.0020 & 15.64 & 15.60 & .99795 & 20 & 40 & .2022 & .2065 & 10211 & 4.945 & 4.843 & .97934 & 20 \\
\hline so & 0869 & .0670 & 1.0022 & 14.96 & 14.92 & 99776 & 10 & 50 & .2051 & 2095 & 1.0217 & 4.877 & 4.773 & .97875 & 10 \\
\hline 4 & .0698 & .0699 & 1.0024 & 14.34 & 14.30 & .99756 & 86 & 12 & 2079 & .2126 & 1.0223 & $4.8 \mathrm{i} 0$ & 4.705 & .97815 & 78 \\
\hline 10 & .0727 & 0729 & 1.0027 & 13.76 & 13.73 & .99736 & 50 & 10 & .2108 & .2156 & 1.0230 & 4.745 & 4.638 & .97754 & so \\
\hline 20 & .0756 & .0758 & 1.0029 & $\mid 13.23$ & 13.20 & .99714 & 40 & 20 & .2136 & .2186 & 1.0236 & 4.682 & 4.574 & .97692 & 40 \\
\hline 30 & .0785 & .0787 & 1.0031 & 12.75 & 12.71 & .99692 & 30 & 30 & .2164 & 2217 & 1.0243 & 4.620 & 4.511 & .97630 & 30 \\
\hline 40 & .0814 & .0816 & 1.0033 & 12.29 & 12.25 & .99668 & 20 & 40 & .2193 & .2247 & 1.0249 & 4560 & 4.449 & .97566 & 20 \\
\hline 50 & .0843 & .0846 & 1.0036 & 11.87 & 11.83 & .99644 & 10 & 50 & .2221 & .2278 & 1.0256 & 4.502 & 4.390 & .97502 & 10 \\
\hline 5 & .0872 & .0875 & 1.0038 & 11.47 & 11.43 & .99619 & 85 & 13 & .2250 & .2309 & 1.0263 & 14.445 & 4.331 & .97437 & 77 \\
\hline 10 & .0901 & .0904 & 1.0041 & 11.10 & 11.06 & .99594 & 50 & 10 & .2278 & .2339 & 1.0270 & 4.390 & 4.275 & .97371 & so \\
\hline 20 & .0929 & .0934 & 1.0043 & 10.76 & 10.71 & .99567 & 40 & 20 & .2306 & .2370 & 1.0277 & 4.336 & 4.219 & .97304 & 40 \\
\hline 30 & .0958 & .0963 & 1.0046 & 10.43 & 10.39 & .99040 & 30 & 30 & .2334 & .2401 & 1.0284 & 4.284 & 4.165 & .97237 & 30 \\
\hline 40 & .0987 & .0992 & 1.0049 & 10.13 & 10.08 & .99511 & 20 & 40 & .2363 & .2432 & 1.0291 & 4.232 & 4.113 & .97169 & 20 \\
\hline 50 & .1016 & .1022 & 1.0052 & 9.839 & 9.788 & .99482 & 10 & 50 & .2391 & .2462 & 1.0299 & 4.182 & 4.061 & .97100 & 10 \\
\hline 6 & .1045 & .1051 & & & 9.514 & .99452 & 84 & 14 & .2419 & .2493 & & & & & 76 \\
\hline 10 & .1074 & .1080 & 0058 & 9.309 & 9.255 & .99421 & 50 & 10 & .2447 & .2524 & 1.0 & 80 & 3.962 & .96959 & 50 \\
\hline 20 & .1103 & .1110 & 1.0061 & 9.065 & 9.010 & .99390 & 40 & 20 & .2470 & .2555 & 1.0321 & 4.039 & 3.914 & .90887 & 40 \\
\hline 30 & .1132 & .1139 & 065 & 8.834 & 8.777 & .99357 & 30 & 30 & .2504 & .2580 & 1.0329 & 3.994 & 3.867 & .96815 & 30 \\
\hline 40 & .1161 & .1169 & 068 & 8.614 & 8.556 & .99324 & 20 & 40 & .2532 & .2617 & 1.0337 & 3.949 & 3.821 & .96742 & 20 \\
\hline so & .1190 & .1198 & 10072 & 8.405 & 8.345 & .99290 & 10 & 50 & .2560 & .2648 & 1.0345 & 3.906 & 3.776 & .96607 & 10 \\
\hline 7 & .1219 & .1228 & 075 & 6.200 & 8.144 & .99255 & 83 & 15 & .2588 & .2679 & 1.0353 & 3.864 & 3.732 & .96593 & 75 \\
\hline 10 & .1248 & .1257 & 1.0079 & 8.016 & 7.953 & .99219 & so & 10 & .2016 & | 2711 & 1.0361 & 3.822 & 3.689 & .96517 & so \\
\hline 20 & 276 & .1287 & 882 & .834 & 7.770 & .99182 & 40 & 20 & .2644 & .2742 & 1.0369 & 3.782 & 3647 & .96440 & 40 \\
\hline 30 & .1305 & .1317 & 1.0086 & 7.661 & 7.596 & .99144 & 30 & 30 & .2672 & .2773 & 1.0377 & 3.742 & 3.606 & .96363 & 30 \\
\hline 40 & $\mid .1334$ & .1346 & & 7.490 & 7.429 & .99106 & 20 & 40 & .2700 & 2805 & 1.0386 & 3.703 & 3.560 & .96285 & 20 \\
\hline so & .1363 & .1376 & 1.0094 & 7.337 & 7.269 & .99067 & 10 & 50 & .2728 & .2836 & 1.0394 & 3.665 & 3.526 & .96200 & 10 \\
\hline & & & & & & & 82 & & & & & & & & 74 \\
\hline & sin & Colg. & Cosec. & Sec. & Ton. & Sin. & Angle & & cosin. & Cotg. & cosec. & sec. & Ion. & sin. & Angle \\
\hline
\end{tabular}


TABLE IV CONTD. NATURAL TRIGONOMETRICAL FUNCTIONS

\begin{tabular}{|c|c|c|c|c|c|c|c|c|c|c|c|c|c|c|c|}
\hline Angle & Sin. & Ton. & Sec. & Cosec. & cotg. & Cosin. & & Anglo & Sin. & Ton. & Sec. & Coser. & cotg. & $\cos i n$. & \\
\hline ' & & & & & & & & 01 & & & & & & & \\
\hline 16 & .2756 & .2867 & 1.0403 & 3.628 & 3.487 & .96126 & 74 & 24 & 4087 & .4452 & 1.0946 & 2.459 & 2.246 & .91355 & 66 \\
\hline 10 & .2784 & .2899 & 1.0412 & 3.592 & 3.450 & 96046 & 50 & 10 & .4094 & .4487 & 10961 & 2.443 & 2.229 & 91236 & 50 \\
\hline 20 & .2812 & 2931 & 1.0423 & 3.556 & 3.412 & .95964 & 40 & 20 & .4120 & .4522 & 1.0975 & 2.427 & 2.211 & .91116 & 40 \\
\hline 30 & .2840 & .2962 & 1.0429 & 3.521 & 3.376 & 95882 & 30 & 30 & .4147 & .4557 & 1.0989 & 2.411 & 2.194 & 90990 & 30 \\
\hline 40 & .2868 & 2994 & 1.0438 & 3.487 & 3.340 & .95799 & 20 & 40 & .4173 & 4592 & 1.1004 & 2.396 & 2.177 & 90875 & 20 \\
\hline 50 & .2896 & .3026 & 1.0448 & 3.453 & 3.305 & .95715 & 10 & 50 & 4200 & .4628 & 11019 & 2.381 & 2.101 & 90753 & 10 \\
\hline 17 & .2924 & .3057 & 1.0457 & 3.420 & 3.271 & .95630 & 73 & 25 & .4226 & .4883 & 1.1034 & 2.366 & 2.145 & .90631 & 65 \\
\hline 10 & .2952 & .3089 & 10466 & 3.388 & 3.237 & 95545 & 50 & 10 & .4253 & 4699 & 1.1049 & 2.351 & 2.128 & 90507 & so \\
\hline 20 & .2979 & 3121 & 1.0476 & 3.357 & 3204 & .95459 & 40 & 20 & .4279 & .4734 & 1.1064 & 2.337 & $2,1+2$ & .90383 & 40 \\
\hline 30 & .3007 & .3153 & 1.0485 & 3.326 & 3.172 & 95372 & 30 & 30 & .4305 & .4770 & 1.1079 & 2.323 & 2.097 & .90259 & 30 \\
\hline 40 & .3035 & 3185 & 1.0495 & 3295 & 3.140 & .95284 & 20 & 40 & .4331 & .4806 & 1.1095 & 2.309 & 2.081 & .90133 & 20 \\
\hline 50 & .3062 & .3217 & 1.0505 & 3.265 & 3.108 & .95195 & 10 & 50 & .4358 & .4841 & 1.1110 & 2.295 & 2060 & 90007 & 10 \\
\hline 18 & .3095 & .3249 & 1.0515 & 3.236 & 3.078 & .95106 & 72 & 26 & 4384 & .4877 & 1.1126 & 2.281 & 2.050 & .89879 & 64 \\
\hline 10 & .3118 & .3281 & 1.0525 & 3.207 & 3.048 & 95015 & so & 10 & .4410 & .4913 & 11142 & 2.268 & 2.035 & .89752 & 50 \\
\hline 20 & .3145 & 3314 & 1.0535 & 3.179 & 3018 & 94924 & 40 & 20 & .4436 & .4950 & 1.1158 & 2.254 & 2.020 & .89623 & 40 \\
\hline 30 & .3173 & .3346 & 1.0545 & 3.152 & 2.989 & .94832 & 30 & 30 & .4462 & 4986 & 1.1174 & 2.241 & 2.006 & .89493 & 30 \\
\hline 40 & .3201 & .3378 & 1.0555 & 3.124 & 2.960 & .94740 & 20 & 40 & .4488 & .5022 & 1.1190 & 2.228 & 1.991 & .89383 & 20 \\
\hline 50 & .3228 & .3411 & 1.0566 & 3.098 & 2.932 & .94646 & 10 & 50 & .4514 & $.50 \leqslant 9$ & 1.1207 & 2.215 & 1.977 & .89232 & 10 \\
\hline 19 & .3256 & .3443 & 1.0576 & 3.072 & 2.904 & .94552 & 71 & 27 & 4540 & .5095 & 1.1223 & 2.203 & 1.963 & .89101 & 83 \\
\hline 10 & .3283 & .3476 & 1.0587 & 3.046 & 2.877 & .94457 & 50 & 10 & 4566 & .5132 & 1.1240 & $2-190$ & 1.949 & 88908 & 50 \\
\hline 20 & .3311 & .3508 & 1.0598 & 3.020 & 2.850 & .94301 & 40 & 20 & 4592 & .5169 & 1.1257 & 2.178 & 1.935 & 335 & 40 \\
\hline 30 & .3338 & .3541 & 1.0608 & 2.996 & 2.824 & .94264 & 30 & 30 & 4617 & .5200 & 1,1274 & 2.188 & 1.921 & 88701 & 30 \\
\hline 40 & .3365 & .3574 & 1.0619 & 2.971 & 2.798 & .94167 & 20 & 40 & 4643 & .5243 & 1.1291 & 2.154 & 1.907 & .88566 & 20 \\
\hline 50 & .3393 & .3607 & 1.0631 & 2.947 & 2.773 & .94068 & 10 & 50 & 4669 & .5280 & 1.1308 & 2.142 & 1.894 & .88431 & 10 \\
\hline 20 & .3420 & .3640 & $|1.0642|$ & 2.924 & 2.747 & .93969 & 70 & 28 & .4695 & .5317 & 1.1326 & 2.130 & 1.881 & .88295 & 62 \\
\hline 10 & .3448 & 3673 & 1.0653 & 2900 & 2.723 & 93869 & 50 & 10 & .4720 & .5354 & 343 & 2.119 & 1.868 & .88158 & 50 \\
\hline 20 & .3475 & 3706 & 1.0685 & 2.878 & 2.699 & 93709 & 40 & 20 & .4746 & .5392 & 1.1381 & 2.107 & 1.855 & .88020 & 40 \\
\hline 30 & .3502 & .3739 & 1.0676 & 2856 & 2.675 & 93607 & 30 & 30 & .4772 & .5430 & 1.1379 & 996 & 1.842 & .87882 & 30 \\
\hline 40 & .3529 & .3772 & $1.068 \mathrm{~B}$ & 2.833 & 2.651 & .93565 & 20 & 40 & .4797 & .5467 & 1.1397 & 2.085 & 1.829 & 87743 & 20 \\
\hline 50 & .3557 & .3805 & 1.0700 & 2.811 & 2.028 & 93462 & 10 & 50 & 4823 & .5505 & 1.1415 & 2.073 & 1.816 & .87603 & 10 \\
\hline 21 & .3584 & .3839 & 1.0711 & $2.790 \mid$ & 2.605 & 93358 & 69 & 29 & .4848 & .5543 & 11434 & 2.063 & $|1.804|$ & 1.87462 & 61 \\
\hline 10 & .3611 & .3872 & 1.0723 & 2.769 & 2.583 & 93253 & 50 & 10 & 4874 & .5581 & 1.1452 & 2.052 & 1.792 & .87321 & 50 \\
\hline 20 & .3638 & .3906 & 1.0736 & 2.749 & 2.560 & .93148 & 40 & 20 & 4899 & .5019 & 1.1471 & .041 & 1.780 & .87178 & 40 \\
\hline 30 & .3665 & .3939 & 1.0748 & 2.729 & 2.539 & .93042 & 30 & 30 & 4924 & .5658 & 1.1490 & 31 & 1.767 & .87036 & 30 \\
\hline 40 & .3692 & .3973 & 1.0760 & 2.709 & 2.517 & .92935 & 20 & 40 & 4950 & .5690 & 1.1509 & 2.020 & 1.756 & .86892 & 20 \\
\hline 50 & .3719 & .4006 & 10773 & 2.689 & 2.490 & .92827 & 10 & 50 & 4975 & .5735 & 11528 & 2.010 & 1.744 & 86748 & 10 \\
\hline 22 & .3746 & .4040 & 1.0785 & 2.670 & 2.475 & .92718 & 68 & 30 & 5000 & .5774 & 11547 & .000 & 1.732 & & $\infty$ \\
\hline 10 & .3773 & .4074 & 1.0798 & 2.650 & 2.455 & .92609 & 50 & 10 & 5025 & .5812 & 1.1506 & 990 & 1.720 & 457 & 50 \\
\hline 20 & .3800 & .4108 & 1.0811 & 2.632 & 2434 & .92499 & 40 & 20 & 5050 & .5851 & 1.1586 & 1.980 & 1.709 & .86310 & 40 \\
\hline 30 & .3827 & 4142 & 1.0824 & 2.613 & 2.414 & .92388 & 30 & 30 & 5075 & .5890 & 1.1606 & 1.970 & 1.698 & 86163 & 30 \\
\hline 40 & .3854 & 4170 & 1.0837 & 2.595 & 2.394 & .92276 & 20 & 40 & 5100 & .5930 & 1.1826 & $1.961 \mid$ & 1.686 & .86015 & 20 \\
\hline 50 & .3881 & .4210 & 1.0850 & 2.577 & 2.375 & 92164 & 10 & 50 & 5125 & 5969 & 1.1646 & 1.951 & 1.675 & .85866 & 10 \\
\hline 23 & .3907 & .4245 & 1.0864 & 2.559 & 2.356 & .92050 & 67 & 31 & 5150 & .6009 & 66 & 24 & 1.604 & .85717 & 59 \\
\hline 10 & .3934 & .4279 & 1.0877 & 2.542 & 2.337 & 91930 & 50 & 10 & 5175 & .6048 & 11687 & 1.932 & 1.053 & 85567 & 50 \\
\hline 20 & .3961 & .4314 & 1.0891 & 2.525 & 2.318 & 91822 & 40 & 20 & 5200 & .0088 & 1.1707 & 1.923 & 1.643 & .85416 & 40 \\
\hline 30 & .3987 & .4348 & 1.0904 & 2.508 & 2.300 & .91706 & 30 & 30 & 5225 & .6128 & 1.1728 & 1.914 & 1.632 & 85264 & 30 \\
\hline 40 & .4014 & .4383 & 1.0918 & 2.491 & 2.282 & 91590 & 20 & 40 & 5250 & .6168 & 1.1749 & 1.905 & 1.621 & 85112 & 20 \\
\hline \multirow[t]{3}{*}{50} & .4041 & .4417 & 1.0932 & 2.475 & 2.264 & 91472 & 10 & 50 & 5275 & .6208 & 1.1770 & 1.896 & 1.611 & .84959 & 10 \\
\hline & & & & & & & 66 & & & & & & & & $5_{0}^{58}$ \\
\hline & cosin. & Ig. & & c. & Ton. & & & & & Corg. & cosec & Sec. & Ion. & Sin. & \\
\hline
\end{tabular}


TABLE IV CONTD. NATURAL TRIGONOMETRICAI, FUNCTIONS

\begin{tabular}{|c|c|c|c|c|c|c|c|c|c|c|c|c|c|c|c|}
\hline Angla & Sin. & Ton. & Soc. & Cosec. & Cotg. & Cosin. & & Anqle & Sin. & Ian & Sec. & Cosec. & Cotg. & Cosin. & \\
\hline - & & & & & & & 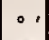 & $\circ \cdot$ & & & & & & & 01 \\
\hline 32 & .5299 & .6249 & 1.1792 & 1.887 & 1.600 & 84805 & 58 & 39 & .6293 & .8098 & 1.2868 & 1.589 & 1.235 & .77715 & 51 \\
\hline 10 & .5324 & .6289 & 1.1813 & 1.878 & 1.590 & 84650 & 50 & 10 & .0318 & .8146 & 1.2898 & 1.583 & 1.228 & .77531 & 50 \\
\hline 20 & .5348 & .6330 & 1.1835 & 1.870 & 1.580 & 84495 & 40 & 20 & .6338 & .8195 & 1.2929 & 1.578 & 1.220 & .77347 & 40 \\
\hline 30 & .5373 & .6371 & 1.1857 & 1.861 & 1.570 & .84339 & 30 & 30 & .0361 & 8243 & 1.2959 & 1.572 & 1.213 & .77162 & 30 \\
\hline 40 & .5398 & .0412 & 1.1879 & 1.853 & 1.560 & .84182 & 20 & 40 & .6383 & .8292 & 1.2991 & 1.567 & 1.206 & .76977 & 20 \\
\hline 50 & .5422 & .0453 & 1.1901 & 1.844 & 1.550 & .84025 & 10 & 50 & .6406 & .8342 & 1.3022 & 1.581 & 1,199 & .76791 & 10 \\
\hline 33 & .5446 & .6494 & 1.1924 & 1.836 & 1.540 & .83867 & 57 & 40 & .0428 & .8391 & 1.3054 & 1,550 & 1,192 & .76604 & 50 \\
\hline 10 & .5471 & .0536 & 11946 & 1.828 & 1.530 & .83708 & 50 & 10 & .6450 & .8441 & 1.3086 & 1.550 & 1.185 & .76417 & 50 \\
\hline 20 & .5495 & .0577 & 1.1969 & 1.820 & 1.520 & .83549 & 40 & 20 & .6472 & .8491 & 1.3118 & 1.545 & 1.178 & .70229 & 40 \\
\hline 30 & .5519 & .6619 & 1.1992 & 1.812 & 1.511 & 83389 & 30 & 30 & .0494 & .8541 & 1.3151 & 1.540 & 1.171 & .76041 & 30 \\
\hline 40 & .5544 & .0601 & 1.2015 & 1.804 & 1.501 & .83228 & 20 & 40 & .0517 & .8591 & 1.3184 & 1.535 & 1.164 & .75851 & 20 \\
\hline 50 & .5568 & .6703 & 12039 & 1.796 & 1.492 & 83060 & 10 & 50 & .6539 & .8642 & 1.3217 & 1.529 & 1.157 & .75661 & 10 \\
\hline 34 & .5592 & .6745 & 1.2062 & 1.788 & 1.483 & .82904 & 56 & 41 & .6561 & .8693 & 13251 & 1.524 & 1.150 & .75471 & 49 \\
\hline 10 & .5616 & .6787 & 1.2086 & 1.781 & 1.473 & .82741 & 50 & 10 & .6583 & .8744 & 1.3284 & 1.519 & 1.144 & .75280 & 50 \\
\hline 20 & .5640 & .6830 & 1.2110 & 1.773 & 1.464 & .82577 & 40 & 20 & .0004 & .8796 & 1.3318 & 1,514 & 1.137 & .75088 & 40 \\
\hline 30 & .5604 & .6873 & 12134 & 1.760 & 1.455 & .82413 & 30 & 30 & .6626 & .8847 & 1.3352 & 1.509 & 1.130 & .74890 & 30 \\
\hline 40 & .5688 & 6916 & 1.2158 & 1.758 & 1.446 & .82248 & 20 & 40 & .6648 & 8899 & 1.3386 & 1.504 & 1.124 & .74703 & 20 \\
\hline 50 & .5712 & .0959 & 1.2183 & 1.751 & 1.437 & .82082 & 10 & 50 & .6670 & .8952 & 13421 & 1.499 & 1.117 & 74509 & 10 \\
\hline 35 & .5736 & .7002 & 1.2208 & 1.743 & 1.428 & .81915 & 55 & 42 & .6091 & .9004 & 1,3456 & 1.494 & 1.111 & .74314 & 48 \\
\hline 10 & .5760 & .7046 & 1.2233 & 1.736 & 1.419 & .81748 & 50 & 10 & .6713 & .9057 & 1.3492 & 1.490 & 1.104 & .74120 & 50 \\
\hline 20 & .5783 & .7089 & 1.2258 & 1.729 & 1.411 & .81580 & 40 & 20 & .6734 & .9110 & 1.3527 & 1.485 & 1.098 & .73924 & 40 \\
\hline 30 & .5807 & .7133 & 12283 & 1.722 & 1.402 & .81412 & 30 & 30 & .6750 & .9163 & 1.3503 & 1.480 & 1.091 & .73728 & 30 \\
\hline 40 & .5831 & .7177 & 1.2309 & 1,715 & 1.393 & .81242 & 20 & 40 & .6777 & .9217 & 1.3600 & 1.470 & 1.085 & .73531 & 20 \\
\hline 50 & .5854 & .7221 & 1.2335 & 1.708 & 1.385 & 81072 & 10 & 50 & .0799 & .9271 & 1,3636 & 1.471 & 1.079 & .73333 & 10 \\
\hline 30 & .5878 & .7265 & 1.2361 & 1.701 & 1.376 & .80902 & 54 & 43 & .6820 & .9325 & 1.3673 & 1.466 & 1.072 & .73135 & 47 \\
\hline 10 & .5901 & .7310 & 1.2387 & 1.695 & 1.368 & .80730 & so & 10 & .6841 & .9380 & 1,3711 & 1,462 & 1.066 & 72937 & 50 \\
\hline 20 & .5925 & .7355 & 1.2413 & 1.088 & 1.360 & .80558 & 40 & 20 & .6862 & .9435 & 1.3748 & 1.457 & 1.000 & .72737 & 40 \\
\hline 30 & .5948 & .7400 & 12440 & 1.681 & 1.351 & 80386 & 30 & 30 & .6884 & .9490 & 1.3786 & 1453 & 1.054 & .72537 & 30 \\
\hline 40 & .5972 & .7445 & 1.2466 & 1.675 & 1.343 & .80212 & 20 & 40 & .6905 & .9545 & 1.3824 & 1.448 & 1.048 & .72337 & 20 \\
\hline 50 & .5995 & .7490 & 12494 & 1.668 & 1.335 & .80038 & 10 & 50 & .6926 & .9601 & 1,3863 & 1.444 & 1.042 & .72136 & 10 \\
\hline 37 & .0018 & .7536 & 1.2521 & 1.662 & 1.327 & .79864 & 53 & 44 & .0947 & .9057 & 1.3902 & 1.440 & 1.036 & .71934 & 46 \\
\hline 10 & .6041 & .7581 & 12549 & 1.655 & 1.319 & .79688 & 50 & 10 & .6967 & .9713 & 13941 & 1.435 & 1.030 & .71732 & 50 \\
\hline 20 & .0065 & .7627 & 1.2577 & 1.649 & 1.311 & .79512 & 40 & 20 & .0988 & .9770 & 1.3980 & 1431 & 1.024 & .71529 & 40 \\
\hline 30 & .6088 & .7673 & 1.2605 & 1.643 & 1.303 & .79335 & 30 & 30 & .7009 & .9827 & 1.4020 & 1.427 & 1.018 & .71325 & 30 \\
\hline 40 & .6111 & .7720 & 1.2633 & 1.636 & 1.295 & .79158 & 20 & 40 & .7030 & .9884 & 1.4061 & 1.422 & 1.012 & .71121 & 20 \\
\hline 50 & .6134 & .7760 & 1.2601 & 1.630 & 1.288 & .78980 & 10 & 50 & .7050 & .9942 & 1.4101 & 1.418 & 1.008 & .70916 & 10 \\
\hline 38 & .6157 & .7813 & 1.2690 & 1.624 & 1.280 & .78801 & 52 & & .7071 & 1. & 1.414 & 1.414 & 1. & .70711 & 45 \\
\hline 10 & .6180 & .7860 & 1.2719 & 1.618 & 1.272 & .78622 & 50 & & & & & & & & \\
\hline 20 & .6202 & .7907 & 1.2748 & 1.012 & 1.265 & .78442 & 40 & & & & & & & & \\
\hline 30 & .6225 & .7954 & 1.2778 & 1.606 & 1.257 & .78261 & 30 & & & & & & & & \\
\hline 40 & .6248 & .8002 & 1.2808 & 1.601 & 1.250 & .78079 & 20 & & & & & & & & \\
\hline \multirow[t]{2}{*}{ so } & .6271 & .8050 & 1.2838 & 1.595 & 1.242 & .77897 & 10 & & & & & & & & \\
\hline & Cosin. & C & Cosec & Sec. & Ton. & Sin. & Angle & & Co & Cot & Cosec. & Sec. & Ton. & Sin. & Angle \\
\hline
\end{tabular}




\section{CURVE FORMULAE \\ CURVE TABLE}

Table of Tangent and External to a $1^{\circ}$ Curve

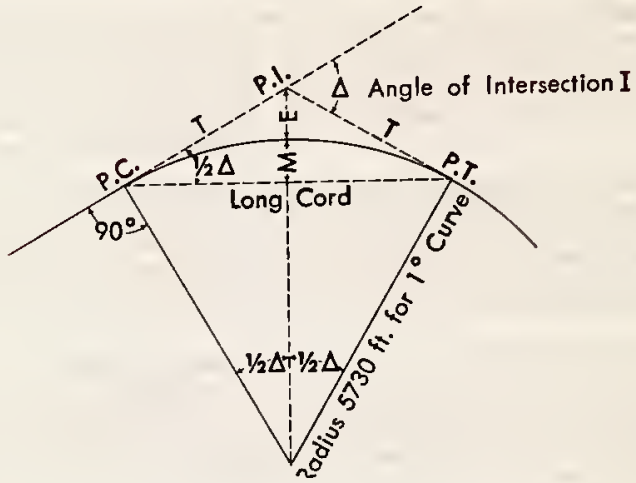

To find Tangent and External for curve of any other degree, divide by degree of curve and add correction found in column of corrections.

Degree of curve with a given I may be found by dividing tangent, (or external), opposite I by given tangent, (or external).

The distance from a point on the tangent to the curve is very nearly the square of the tangent length divided by twice the radius.

\section{CURVE FORMULAS}

Radius:

$$
\mathrm{R}=\frac{50}{\sin 1 / 2 \mathrm{D}}
$$

Length of Curve: $\mathrm{L}=100 \frac{\Delta}{\mathrm{D}}$

$$
\text { also } \mathrm{L}=.0174533 \times \Delta \times \mathrm{R}
$$

Degree of Curve: $\mathrm{D}=100 \frac{\Delta}{\mathrm{L}}$

Tangent: $\quad \mathrm{T}=\mathrm{R} \tan 1 / 2 \Delta$

Long Cord: $\quad L C=2 R \sin 1 / 2 \Delta$

Middle Ordinate: $\mathrm{M}=\mathrm{R}(1-\operatorname{Cos} 1 / 2 \Delta)$

External: $\quad E=T \tan 1 / 4 \Delta$ 
TABLE V. TANGENTS AND EXTERNALS TO A $1^{\circ}$ CURVE

\begin{tabular}{|c|c|c|c|c|c|c|c|c|c|c|c|}
\hline$I$ & $T$ & E & $I=10^{\circ}$ & $I$ & $\mathrm{~T}$ & E & $=20^{\circ}$ & $I$ & $T$ & E & \\
\hline $\begin{array}{l}10^{\prime} \\
20^{\prime} \\
30^{\prime} \\
40^{\prime} \\
50^{\prime}\end{array}$ & $\begin{array}{l}50.00 \\
58.34 \\
66.67 \\
75.01 \\
83.34 \\
91.68\end{array}$ & $\begin{array}{l}.218 \\
.297 \\
.388 \\
.491 \\
.606 \\
.733\end{array}$ & $\begin{array}{c}+ \\
5^{\circ} \mathrm{C} . \\
\mathrm{T} \\
.03 \\
E\end{array}$ & $\begin{array}{r}110 \\
10 \\
20 \\
30 \\
40 \\
50\end{array}$ & $\begin{array}{l}551.70 \\
560.11 \\
568.53 \\
576.95 \\
585.36 \\
593.79\end{array}$ & & $\begin{array}{c}+ \\
5^{\circ} \mathrm{C} . \\
T \\
.06 \\
E\end{array}$ & $\begin{array}{r}21^{\circ} \\
10 \\
20 \\
30 \\
40^{\prime} \\
50\end{array}$ & & & $\begin{array}{l}+ \\
5^{\circ} \mathrm{C} . \\
\mathrm{T} \\
.10 \\
\mathrm{E}\end{array}$ \\
\hline $\begin{array}{c}2^{\circ} \\
10 \\
20^{\prime} \\
30^{\prime} \\
40^{\prime} \\
50^{\prime}\end{array}$ & $\begin{array}{l}100.01 \\
108.35 \\
116.68 \\
125.02 \\
133.36 \\
141.70\end{array}$ & $\begin{array}{r}.873 \\
1.024 \\
1.188 \\
1.364 \\
1.552 \\
1.752\end{array}$ & .001 & $\begin{array}{r}12 \\
10 \\
20 \\
30 \\
40 \\
50\end{array}$ & $\begin{array}{l}602 \\
610 \\
619 \\
627 \\
635 \\
644\end{array}$ & & .006 & $\begin{array}{r}22^{\circ} \\
10^{\prime} \\
20^{\prime} \\
30^{\prime} \\
40^{\prime} \\
50^{\prime}\end{array}$ & $\begin{array}{l}1113.7 \\
1122.4 \\
1131.0 \\
1139.7 \\
1148.4 \\
1157.0\end{array}$ & & .013 \\
\hline $\begin{array}{l}10^{\prime} \\
20^{\prime} \\
30^{\prime} \\
40^{\prime} \\
50^{\prime}\end{array}$ & $\begin{array}{l}15 \\
15 \\
16 \\
17 \\
18 \\
19\end{array}$ & $\begin{array}{l}1.9 \\
2.1 \\
2.4 \\
2.6 \\
2.9 \\
3.2\end{array}$ & $\begin{array}{c}10^{\circ} \mathrm{C} . \\
\mathrm{T} \\
.06 \\
\mathrm{E} \\
.003\end{array}$ & $\begin{array}{r}13^{\circ} \\
10 \\
20 \\
30 \\
40 \\
50\end{array}$ & & & $\begin{array}{c}10^{\circ} \mathrm{C} . \\
\mathrm{T} \\
.13 \\
\mathrm{E} \\
.011\end{array}$ & $\begin{array}{r}23^{\circ} \\
10^{\prime} \\
20^{\prime} \\
30^{\prime} \\
40^{\prime} \\
50^{\prime}\end{array}$ & & & $\begin{array}{c}10^{\circ} \mathrm{C} . \\
\mathrm{T} \\
.19 \\
\mathrm{E} \\
.025\end{array}$ \\
\hline $\begin{array}{l}10^{\prime} \\
20^{\prime} \\
30^{\prime} \\
40^{\prime} \\
50^{\prime}\end{array}$ & $\begin{array}{l}200.08 \\
208.43 \\
216.77 \\
225.12 \\
233.47 \\
241.81\end{array}$ & $\begin{array}{l}3.4 \\
3.7 \\
4.0 \\
4.4 \\
4.7 \\
5.1\end{array}$ & $5^{\circ} \mathrm{C}$. & $\begin{array}{r}14^{\circ} \\
10 \\
20 \\
30^{\circ} \\
40^{\circ} \\
50^{\prime}\end{array}$ & & & 150 & $\begin{array}{r}24^{\circ} \\
10^{\prime} \\
20^{\prime} \\
30^{\prime} \\
40^{\prime} \\
50^{\prime}\end{array}$ & & & $13^{\circ} \mathrm{C}$ \\
\hline $\begin{array}{l}10^{\prime} \\
20^{\prime} \\
30^{\prime} \\
40^{\prime} \\
50^{\prime}\end{array}$ & $\begin{array}{l}2 \\
2 \\
2 \\
2 \\
2 \\
2\end{array}$ & $\begin{array}{l}5.459 \\
5.829 \\
6.211 \\
6.606 \\
7.013 \\
7.432\end{array}$ & $\begin{array}{c}T \\
.09 \\
E \\
.004\end{array}$ & $\begin{array}{r}15^{\circ} \\
10 \\
20^{\prime} \\
30^{\prime} \\
40^{\prime} \\
50^{\circ}\end{array}$ & & & $\begin{array}{c}\mathrm{T} \\
.19 \\
\mathrm{E} \\
.017\end{array}$ & $\begin{array}{l}15^{\circ} \\
10^{\prime} \\
30 \\
40 \\
50\end{array}$ & & & $\begin{array}{c}T \\
.29 \\
E \\
.038\end{array}$ \\
\hline $\begin{array}{l}10^{\prime} \\
20^{\prime} \\
30^{\prime} \\
40^{\prime} \\
50^{\prime}\end{array}$ & $\begin{array}{l}3 \\
3 \\
3 \\
3 \\
3\end{array}$ & $\begin{array}{r}7.8 \\
8.3 \\
8.7 \\
9.2 \\
9.7 \\
10.2\end{array}$ & $20^{\circ} \mathrm{C}$. & $\begin{array}{r}16^{\circ} \\
10 \\
20 \\
30 \\
40^{\circ} \\
50^{\prime}\end{array}$ & & $\begin{array}{l}5 \\
5 \\
5 \\
5 \\
6 \\
6\end{array}$ & $20^{\circ} \mathrm{C}$. & $\begin{array}{r}26^{\circ} \\
10^{\prime} \\
20^{\prime} \\
30^{\prime} \\
40^{\prime} \\
50^{\prime}\end{array}$ & & & $\begin{array}{c}20^{\circ} \mathrm{C} . \\
\mathrm{T}\end{array}$ \\
\hline $\begin{array}{l}10 \\
20 \\
30 \\
40^{\prime} \\
50^{\prime}\end{array}$ & & $\begin{array}{l}10.707 \\
11.224 \\
11.753 \\
12.294 \\
12.847 \\
13.413\end{array}$ & $\begin{array}{c}\mathrm{E} \\
.006\end{array}$ & $\begin{array}{r}17^{\circ} \\
10^{\prime} \\
20^{\prime} \\
30^{\prime} \\
40^{\prime} \\
50^{\prime}\end{array}$ & & $\begin{array}{l}63 \\
64 \\
66 \\
67 \\
68 \\
70\end{array}$ & $\begin{array}{c}.26 \\
E \\
.022\end{array}$ & $\begin{array}{l}10^{\prime} \\
20^{\prime} \\
30^{\prime} \\
40^{\prime} \\
50^{\prime}\end{array}$ & & & $\begin{array}{c}.39 \\
E \\
.051\end{array}$ \\
\hline $\begin{array}{l}10 \\
20 \\
30 \\
40 \\
50\end{array}$ & & $\begin{array}{l}13 \\
12 \\
15 \\
15 \\
16 \\
17\end{array}$ & $\begin{array}{c}25^{\circ} \mathrm{C} . \\
T \\
.16 \\
E\end{array}$ & $\begin{array}{l}10 \\
20 \\
30 \\
40^{\prime} \\
50^{\prime}\end{array}$ & & & $\begin{array}{c}25^{\circ} \mathrm{C} . \\
T \\
.32 \\
E\end{array}$ & $\begin{array}{r}28^{\circ} \\
10^{\prime} \\
20^{\prime} \\
30^{\prime} \\
40^{\prime} \\
50^{\prime}\end{array}$ & & & $\begin{array}{c}25^{\circ} \mathrm{C} . \\
T \\
.49 \\
E\end{array}$ \\
\hline $\begin{array}{l}10^{\prime} \\
20^{\prime} \\
30^{\prime} \\
40^{\prime} \\
50^{\prime}\end{array}$ & & & .007 & \begin{tabular}{r|}
$19^{\circ}$ \\
$10^{\prime}$ \\
$20^{\prime}$ \\
$30^{\prime}$ \\
$40^{\prime}$ \\
$50^{\prime}$
\end{tabular} & & & .028 & $\begin{array}{r}29^{\circ} \\
10^{\prime} \\
20^{\prime} \\
30^{\prime} \\
40^{\prime} \\
50^{\prime}\end{array}$ & & & .065 \\
\hline $\begin{array}{l}10^{\prime} \\
20 \\
30^{\prime} \\
40^{\prime} \\
50^{\prime}\end{array}$ & $\begin{array}{l}50 \\
518 \\
52 \\
53\end{array}$ & $\begin{array}{l}21 \\
22 \\
23 \\
24 \\
24 \\
25\end{array}$ & $\begin{array}{c}30^{\circ} \mathrm{C} \\
\mathrm{T} \\
19 \\
\mathrm{E} \\
008\end{array}$ & $\begin{array}{r}20^{\circ} \\
10 \\
20 \\
30 \\
40^{\prime} \\
50^{\circ}\end{array}$ & $\begin{array}{l}10 \\
10 \\
10 \\
10 \\
10 \\
10\end{array}$ & & $\begin{array}{c}30^{\circ} \mathrm{C} . \\
\mathrm{T} \\
.39 \\
\mathrm{E} \\
.034\end{array}$ & $\begin{array}{r}30^{\circ} \\
10^{\prime} \\
20^{\prime} \\
30^{\prime} \\
40^{\prime} \\
50^{\prime}\end{array}$ & & & $\begin{array}{c}30^{\circ} \mathrm{C} . \\
\mathrm{T} \\
.59 \\
\mathrm{E} \\
.078\end{array}$ \\
\hline
\end{tabular}


TABLE $V$ CONTD. TANGENTS AND EXTERNALS TO A $1^{\circ}$ CURVE

\begin{tabular}{|c|c|c|c|c|c|c|c|c|c|c|c|}
\hline$I$ & 1 & E & $I=40^{\circ}$ & $I$ & I & E & $I=50^{\circ}$ & $I$ & $T$ & E & $I=60^{\circ}$ \\
\hline $\begin{array}{l}10^{\circ} \\
20^{\prime} \\
30^{\prime} \\
40^{\prime} \\
50^{\prime}\end{array}$ & $\begin{array}{l}1589.0 \\
1598.0 \\
1606.9 \\
1615.9 \\
1624.9 \\
1633.9\end{array}$ & $\begin{array}{l}216.3 \\
218.7 \\
221.1 \\
223.5 \\
226.0 \\
228.4\end{array}$ & $\begin{array}{c}{ }^{+} \mathrm{C} . \\
T \\
13 \\
\mathrm{E}\end{array}$ & $\begin{array}{r}41^{\circ} \\
10^{\prime} \\
20^{\prime} \\
30^{\prime} \\
40^{\prime} \\
50^{\prime}\end{array}$ & $\begin{array}{l}2142.2 \\
2151.7 \\
2161.2 \\
2170.8 \\
2180.3 \\
2189.9\end{array}$ & $\begin{array}{l}387.1 \\
390.7 \\
394.1 \\
397.4 \\
400.8 \\
404.2\end{array}$ & $\begin{array}{c}+ \\
5^{\circ} \mathrm{C} . \\
T \\
.17 \\
\mathrm{E}\end{array}$ & $\begin{array}{r}51^{\circ} \\
10^{\prime} \\
20^{\prime} \\
30^{\prime} \\
40^{\prime} \\
50^{\prime}\end{array}$ & $\begin{array}{l}2732.9 \\
2743.1 \\
2753.4 \\
2763.7 \\
2773.9 \\
2784.2\end{array}$ & $\begin{array}{l}618.4 \\
622.8 \\
627.2 \\
631.7 \\
636.2 \\
640.7\end{array}$ & $\begin{array}{c}+ \\
5^{\circ} \mathrm{C} . \\
\mathrm{T} \\
.21 \\
\mathrm{E}\end{array}$ \\
\hline $\begin{array}{r}32^{\circ} \\
10^{\circ} \\
20^{\circ} \\
30^{\circ} \\
40^{\prime} \\
50^{\prime}\end{array}$ & $\begin{array}{l}1643.0 \\
1652.0 \\
1661.0 \\
1670.0 \\
1679.1 \\
1688.1\end{array}$ & $\begin{array}{l}230.9 \\
233.4 \\
235.9 \\
238.4 \\
241.0 \\
243.5\end{array}$ & .023 & $\begin{array}{r}42^{\circ} \\
10^{\circ} \\
20^{\prime} \\
30^{\prime} \\
40^{\prime} \\
50^{\prime}\end{array}$ & $\begin{array}{l}2199.4 \\
2209.0 \\
2218.6 \\
2228.1 \\
2237.7 \\
2247.3\end{array}$ & $\begin{array}{l}407.6 \\
411.1 \\
414.5 \\
418.0 \\
421.4 \\
425.0\end{array}$ & .037 & $\begin{array}{r}52^{\circ} \\
10^{\prime} \\
20^{\prime} \\
30^{\prime} \\
40^{\circ} \\
50^{\prime}\end{array}$ & $\begin{array}{l}2794.5 \\
2804.9 \\
2815.2 \\
2825.6 \\
2835.9 \\
2846.3\end{array}$ & $\begin{array}{l}645.2 \\
649.7 \\
654.3 \\
658.8 \\
663.4 \\
668.0\end{array}$ & .056 \\
\hline $\begin{array}{r}33^{\circ} \\
10^{\prime} \\
20^{\prime} \\
30^{\circ} \\
40^{\prime} \\
50^{\prime}\end{array}$ & $\begin{array}{l}1697.2 \\
1706.3 \\
1715.3 \\
1724.4 \\
1733.5 \\
1742.6\end{array}$ & $\begin{array}{l}246.1 \\
248.7 \\
251.3 \\
253.9 \\
256.5 \\
259.1\end{array}$ & $\begin{array}{c}10^{\circ} \mathrm{C} \\
\mathrm{T} \\
.26 \\
\mathrm{E} \\
.046\end{array}$ & $\begin{array}{r}43^{\circ} \\
10^{\prime} \\
20^{\prime} \\
30^{\prime} \\
40^{\prime} \\
50^{\prime}\end{array}$ & $\begin{array}{l}2257.0 \\
2266.6 \\
22762 \\
2285.9 \\
2295.6 \\
23052\end{array}$ & $\begin{array}{l}428.5 \\
432.0 \\
435.6 \\
439.2 \\
442.8 \\
446.4\end{array}$ & $\begin{array}{c}10^{\circ} \mathrm{C} . \\
\mathrm{T} \\
.34 \\
\mathrm{E} \\
.075\end{array}$ & $\begin{array}{r}53^{\circ} \\
10^{\circ} \\
20^{\circ} \\
30^{\prime} \\
40^{\circ} \\
50^{\prime}\end{array}$ & $\begin{array}{l}2856.7 \\
2867.1 \\
2877.5 \\
2888.0 \\
2898.4 \\
2908.9\end{array}$ & $\begin{array}{l}672.7 \\
677.3 \\
682.0 \\
686.7 \\
691.4 \\
696.1\end{array}$ & $\begin{array}{c}10^{\circ} \mathrm{C} . \\
\mathrm{T} \\
.42 \\
\mathrm{E} \\
.112\end{array}$ \\
\hline $34^{\circ}$ & $\begin{array}{l}17517 \\
1750.8\end{array}$ & $\begin{array}{l}261.8 \\
264.5\end{array}$ & & $\begin{array}{l}44^{\circ} \\
10^{\circ}\end{array}$ & $\begin{array}{l}2314.9 \\
23246\end{array}$ & $\begin{array}{l}450.0 \\
453.6\end{array}$ & & $\begin{array}{c}54^{\circ} \\
10^{\prime}\end{array}$ & & $\begin{array}{l}700.9 \\
705.7\end{array}$ & \\
\hline $\begin{array}{l}20^{\prime} \\
30^{\prime} \\
40^{\prime} \\
50^{\prime}\end{array}$ & $\begin{array}{l}1770.0 \\
1779.1 \\
1788.2 \\
1797.4\end{array}$ & $\begin{array}{l}267.2 \\
269.9 \\
272.6 \\
275.3\end{array}$ & $12 \mathrm{co}$ & $\begin{array}{l}20^{\prime} \\
30^{\prime} \\
40^{\prime} \\
50^{\prime}\end{array}$ & $\begin{array}{l}2334.3 \\
2344.1 \\
2353.8 \\
2363.5\end{array}$ & $\begin{array}{l}457.3 \\
461.0 \\
464.5 \\
468.4\end{array}$ & 12 & $\begin{array}{l}20^{\circ} \\
30^{\circ} \\
40^{\prime} \\
50^{\prime}\end{array}$ & $\begin{array}{l}2940.4 \\
2951.0 \\
2961.5 \\
2972.1\end{array}$ & $\begin{array}{l}710.5 \\
715.3 \\
720.1 \\
725.0\end{array}$ & $15^{7} \mathrm{C}$ \\
\hline $\begin{array}{r}35^{\circ} \\
10^{\prime} \\
20^{\prime} \\
30^{\prime} \\
40^{\prime} \\
50^{\prime}\end{array}$ & $\begin{array}{l}1806.6 \\
1815.7 \\
1824.9 \\
1834.1 \\
1843.3 \\
1852.5\end{array}$ & $\begin{array}{l}278.1 \\
280.8 \\
283.6 \\
286.4 \\
2892 \\
292.0\end{array}$ & $\begin{array}{c}T \\
.40 \\
E \\
.070\end{array}$ & $\begin{array}{r}45^{\circ} \\
10^{\prime} \\
20^{\circ} \\
30^{\prime} \\
40^{\prime} \\
50^{\prime}\end{array}$ & $\begin{array}{l}2373.3 \\
2383.1 \\
2392.8 \\
2402.6 \\
2412.4 \\
2422.3\end{array}$ & $\begin{array}{l}472.1 \\
475.8 \\
4796 \\
483.4 \\
487.2 \\
491.0\end{array}$ & $\begin{array}{c}T \\
.51 \\
E \\
116\end{array}$ & \begin{tabular}{|r|}
$55^{\circ}$ \\
$10^{\prime}$ \\
20 \\
30 \\
$40^{\prime}$ \\
$50^{\circ}$
\end{tabular} & $\begin{array}{l}2982.7 \\
2993.3 \\
3003.9 \\
3014.5 \\
3025.2 \\
3035.8\end{array}$ & $\begin{array}{l}729.9 \\
734.8 \\
739.7 \\
744.6 \\
749.6 \\
754.6\end{array}$ & $\begin{array}{c}T \\
.63 \\
E \\
.168\end{array}$ \\
\hline $\begin{array}{r}36^{\circ} \\
10^{\prime} \\
20^{\prime} \\
30^{\prime} \\
40^{\circ} \\
50^{\prime}\end{array}$ & $\begin{array}{l}1861.7 \\
1870.9 \\
1880.1 \\
1889.4 \\
1898.6 \\
1907.9\end{array}$ & $\begin{array}{l}294.9 \\
297.7 \\
300.6 \\
303.5 \\
306.4 \\
309.3\end{array}$ & $\frac{20^{\circ} \mathrm{C} .}{T}$ & $\begin{array}{r}46^{\circ} \\
10^{\prime} \\
20^{\prime} \\
30^{\prime} \\
40^{\prime} \\
50^{\prime}\end{array}$ & $\begin{array}{l}2432.1 \\
2441.9 \\
2451.8 \\
2461.7 \\
2471.5 \\
2481.4\end{array}$ & $\begin{array}{l}494.8 \\
498.7 \\
502.5 \\
506.4 \\
510.3 \\
514.3\end{array}$ & $20^{\circ} \mathrm{C}$. & $\begin{array}{r}56^{\circ} \\
10^{\circ} \\
20^{\circ} \\
30^{\circ} \\
40^{\circ} \\
50^{\circ}\end{array}$ & $\begin{array}{l}3046.5 \\
3057.2 \\
3067.9 \\
3078.7 \\
3089.4 \\
3100.2\end{array}$ & $\begin{array}{l}759.6 \\
764.6 \\
769.7 \\
774.7 \\
779.8 \\
784.9\end{array}$ & $\begin{array}{c}20-\mathrm{C} . \\
\mathrm{T} \\
.84\end{array}$ \\
\hline $\begin{array}{r}37^{\circ} \\
10^{\prime} \\
20^{\prime} \\
30^{\prime} \\
40^{\prime} \\
50^{\prime}\end{array}$ & $\begin{array}{l}1917.1 \\
1926.4 \\
1935.7 \\
1945.0 \\
1954.3 \\
1963.6\end{array}$ & $\begin{array}{l}312.2 \\
315.2 \\
318.1 \\
321.1 \\
324.1 \\
327.1\end{array}$ & $\begin{array}{c}.53 \\
E \\
.093\end{array}$ & $\begin{array}{r}47^{\circ} \\
10^{\circ} \\
20^{\circ} \\
30^{\circ} \\
40^{\circ} \\
50^{\prime}\end{array}$ & $\begin{array}{l}24913 \\
2501.2 \\
2511.2 \\
2521.1 \\
2531.1 \\
2541.0\end{array}$ & $\begin{array}{l}518.2 \\
522.2 \\
526.1 \\
530.1 \\
534.2 \\
538.2\end{array}$ & $\begin{array}{c}.68 \\
E \\
.151\end{array}$ & $\begin{array}{r}57^{\circ} \\
10^{\prime} \\
20^{\prime} \\
30^{\prime} \\
40^{\prime} \\
50^{\prime}\end{array}$ & $\begin{array}{l}3110.9 \\
3121.7 \\
3132.6 \\
3143.4 \\
3154.2 \\
3165.1\end{array}$ & $\begin{array}{l}790.1 \\
795.2 \\
800.4 \\
8056 \\
810.9 \\
816.1\end{array}$ & $\begin{array}{c}E \\
.225\end{array}$ \\
\hline $\begin{array}{l}18^{\circ} \\
10^{\prime} \\
20^{\prime} \\
30^{\prime} \\
40^{\prime} \\
50^{\prime}\end{array}$ & $\begin{array}{l}1972.9 \\
1982.2 \\
1991.5 \\
2000.9 \\
2010.2 \\
2019.6\end{array}$ & $\begin{array}{l}330.2 \\
3332 \\
336.3 \\
339.3 \\
342.4 \\
345.5\end{array}$ & $\begin{array}{c}25^{\circ} \mathrm{C} . \\
\mathrm{T} \\
.67 \\
\mathrm{E}\end{array}$ & $\begin{array}{r}48^{\circ} \\
10^{\circ} \\
20^{\prime} \\
30^{\prime} \\
40^{\prime} \\
50^{\prime}\end{array}$ & $\begin{array}{l}2551.0 \\
2561.0 \\
2571.0 \\
2581.0 \\
2591.0 \\
2601.1\end{array}$ & $\begin{array}{l}542.2 \\
546.3 \\
550.4 \\
554.5 \\
558.6 \\
562.8\end{array}$ & $\begin{array}{c}25 \mathrm{C} \\
T \\
85 \\
E\end{array}$ & \begin{tabular}{|r|}
$58^{\circ}$ \\
$10^{\prime}$ \\
20 \\
30 \\
40 \\
$50^{\circ}$
\end{tabular} & $\begin{array}{l}3176.0 \\
3186.9 \\
3197.8 \\
3208.8 \\
3219.7 \\
3230.7\end{array}$ & $\begin{array}{l}821.4 \\
826.7 \\
832.0 \\
837.3 \\
842.7 \\
848.1\end{array}$ & $\begin{array}{c}25^{\circ} \mathrm{C} . \\
T \\
1.05 \\
E\end{array}$ \\
\hline $\begin{array}{l}10^{\prime} \\
20^{\prime} \\
30^{\prime}\end{array}$ & $\begin{array}{l}2029.0 \\
2038.4 \\
2047.8\end{array}$ & $\begin{array}{l}348.6 \\
351.8 \\
354.9\end{array}$ & .117 & $\begin{array}{l}49^{\circ} \\
10^{\circ} \\
20^{\circ}\end{array}$ & $\begin{array}{l}26112 \\
2621.2 \\
2631.3\end{array}$ & $\begin{array}{l}566.9 \\
571.1 \\
575.3\end{array}$ & 189 & $\begin{array}{r}59^{\circ} \\
10^{\prime} \\
20^{\prime}\end{array}$ & $\begin{array}{l}3241.7 \\
3252.7 \\
3263.7\end{array}$ & $\begin{array}{l}853.5 \\
858.9 \\
8643\end{array}$ & .283 \\
\hline $\begin{array}{l}30^{\prime} \\
40^{\prime} \\
50^{\circ}\end{array}$ & $\begin{array}{l}2057.2 \\
2066.6 \\
2076.0\end{array}$ & $\begin{array}{l}358.1 \\
361.3 \\
364.5\end{array}$ & & $\begin{array}{l}30^{\prime} \\
40^{\circ} \\
50^{\circ}\end{array}$ & $\begin{array}{l}2641.4 \\
2651.5 \\
2661.6\end{array}$ & $\begin{array}{l}5795 \\
583.8 \\
5880\end{array}$ & & $\begin{array}{l}30 \\
40 \\
50^{\circ}\end{array}$ & $\begin{array}{l}3274.8 \\
3285.8 \\
3296.9\end{array}$ & $\begin{array}{l}869.8 \\
875.3 \\
880.8\end{array}$ & \\
\hline $\begin{array}{l}0^{\circ} \\
10^{\prime} \\
20^{\prime} \\
30^{\prime} \\
40^{\prime} \\
50^{\prime}\end{array}$ & $\begin{array}{l}2085.4 \\
2094.9 \\
2104.3 \\
2113.8 \\
2123.3 \\
2132.7 \\
\end{array}$ & $\begin{array}{l}367.7 \\
371.0 \\
374.2 \\
377.5 \\
380.8 \\
384.1\end{array}$ & $\begin{array}{c}30^{\circ} \mathrm{C} \\
T \\
.80 \\
E \\
141\end{array}$ & $\begin{array}{r}50^{\circ} \\
10^{\prime} \\
20^{\circ} \\
30^{\circ} \\
40^{\circ} \\
50^{\circ} \\
\end{array}$ & $\begin{array}{l}2671.8 \\
2681.9 \\
2692.1 \\
2702.3 \\
2712.5 \\
2722.7\end{array}$ & $\begin{array}{l}592.3 \\
5966 \\
600.9 \\
605.3 \\
609.6 \\
614.0\end{array}$ & $\begin{array}{c}30^{\circ} \mathrm{C} \\
\mathrm{T} \\
1.02 \\
\mathrm{E} \\
227\end{array}$ & $\begin{array}{r}60^{\circ} \\
10^{\circ} \\
20^{\prime} \\
30^{\circ} \\
40^{\prime} \\
50^{\prime}\end{array}$ & $\begin{array}{l}3308.0 \\
3319.1 \\
3330.3 \\
3341.4 \\
3352.6 \\
3363.8\end{array}$ & $\begin{array}{l}886.4 \\
892.0 \\
897.5 \\
903.2 \\
908.8 \\
914.5\end{array}$ & $\begin{array}{c}30 \mathrm{C} . \\
T \\
1.27 \\
E \\
.340\end{array}$ \\
\hline
\end{tabular}

$T=R \tan 1 / 2 I$

$E=R_{\text {exsec }} 1 / 2 I$ 
TABLE V CONTD. TANGENTS AND EXTERNALS TO A $1^{\circ}$ CURVE

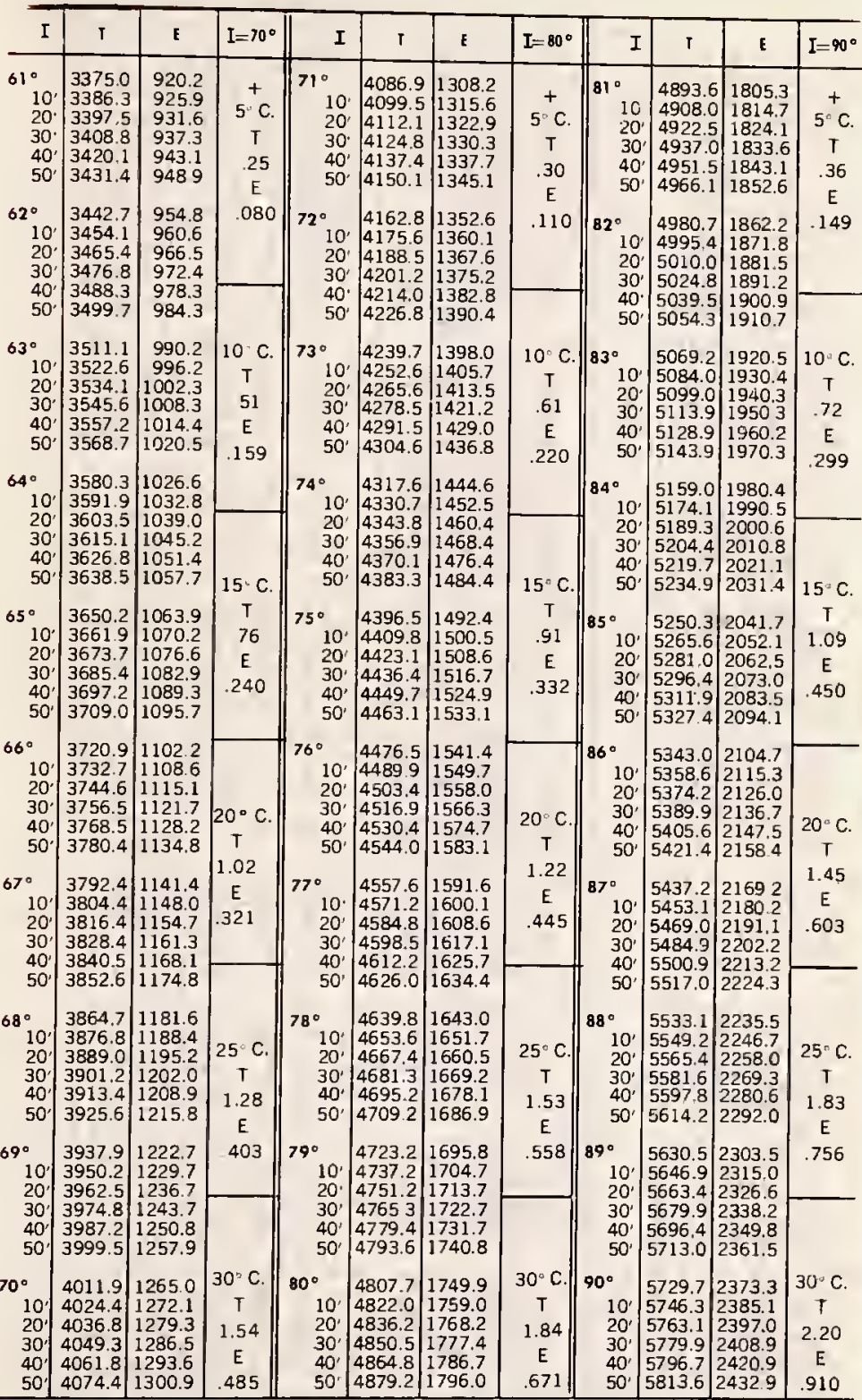


TABI.E V CONTD. TANGENTS AND EXTERNALS TO A $1^{\circ}$ CURVE

\begin{tabular}{|c|c|c|c|c|c|c|c|c|c|c|c|}
\hline$I$ & $T$ & E & $I=100^{\circ}$ & I & $T$ & E & $I=110^{\circ}$ & $I$ & $T$ & $\varepsilon$ & $I=120^{\circ}$ \\
\hline $\begin{array}{r}91^{\circ} \\
10^{\prime} \\
20^{\prime} \\
30^{\prime} \\
40^{\prime} \\
50^{\prime}\end{array}$ & $\begin{array}{l}5830.5 \\
5847.5 \\
5864.6 \\
5881.7 \\
5898.8 \\
5916.0\end{array}$ & $\begin{array}{l}2444.9 \\
2457.1 \\
2469.3 \\
2481.5 \\
2493.8 \\
2506.1\end{array}$ & $\begin{array}{c}+ \\
5 \mathrm{C} . \\
T \\
.43 \\
\mathrm{E}\end{array}$ & $\begin{array}{r}101^{\circ} \\
10 \\
20 \\
30 \\
40 \\
50^{\prime}\end{array}$ & $\begin{array}{l}6950.6 \\
6971.3 \\
6992.0 \\
7012.7 \\
7033.6 \\
7054.5\end{array}$ & \begin{tabular}{|l|}
3278.1 \\
3294.1 \\
3310.1 \\
3326.1 \\
3342.3 \\
3358.5
\end{tabular} & $\begin{array}{c}+ \\
5^{\circ} \mathrm{C} . \\
\mathrm{T} \\
.51 \\
\mathrm{E}\end{array}$ & $\begin{array}{r}111^{\circ} \\
10^{\prime} \\
20^{\prime} \\
30^{\prime} \\
40^{\prime} \\
50^{\prime}\end{array}$ & $\begin{array}{l}8336.7 \\
8362.7 \\
8388.9 \\
8415.1 \\
8441.5 \\
8468.0\end{array}$ & $\begin{array}{l}4386.1 \\
4407.6 \\
4429.2 \\
4450.9 \\
4472.7 \\
4494.6\end{array}$ & $\begin{array}{c}+ \\
5^{\circ} \mathrm{C} . \\
T \\
62 \\
\mathrm{E}\end{array}$ \\
\hline $\begin{array}{r}92^{\circ} \\
10^{\prime} \\
20^{\prime} \\
30^{\prime} \\
40^{\prime} \\
50^{\prime}\end{array}$ & $\begin{array}{l}5933.2 \\
5950.5 \\
5967.9 \\
5985.3 \\
6002.7 \\
6020.2\end{array}$ & $\begin{array}{l}2518.5 \\
2531.0 \\
2543.5 \\
2556.0 \\
2568.6 \\
2581.3\end{array}$ & .200 & $\begin{array}{r}102^{\circ} \\
10^{\circ} \\
20^{\prime} \\
30^{\prime} \\
40^{\prime} \\
50^{\prime}\end{array}$ & $\begin{array}{l}7075.5 \\
7096.6 \\
7117.8 \\
7139.0 \\
7160.3 \\
7181.7\end{array}$ & $\begin{array}{l}3374.9 \\
3391.2 \\
3407.7 \\
3424.3 \\
3440.9 \\
3457.6\end{array}$ & 268 & $\begin{array}{r}112^{\circ} \\
10^{\prime} \\
20^{\prime} \\
30^{\prime} \\
40^{\prime} \\
50^{\prime}\end{array}$ & $\begin{array}{l}8494.6 \\
8521.3 \\
8548.1 \\
8575.0 \\
8602.1 \\
8629.3\end{array}$ & $\begin{array}{l}4516.6 \\
4538.8 \\
4561.1 \\
4583.4 \\
4606.0 \\
4628.6\end{array}$ & .360 \\
\hline $\begin{array}{r}93^{\circ} \\
10^{\prime} \\
20^{\prime} \\
30^{\prime} \\
40^{\prime} \\
50^{\prime}\end{array}$ & $\begin{array}{l}6037.8 \\
6055.4 \\
6073.1 \\
6090.8 \\
6108.6 \\
6126.4\end{array}$ & $\left|\begin{array}{l}2594.0 \\
2606.8 \\
2619.7 \\
2632.6 \\
2645.5 \\
2658.5\end{array}\right|$ & $\begin{array}{c}10^{\circ} \mathrm{C} . \\
\mathrm{T} \\
.86 \\
\mathrm{E} \\
.401\end{array}$ & $\begin{array}{r}103^{\circ} \\
10^{\prime} \\
20^{\prime} \\
30^{\prime} \\
40^{\prime} \\
50^{\prime}\end{array}$ & \begin{tabular}{|l|}
7203.2 \\
7224.7 \\
7246.3 \\
7268.0 \\
7289.8 \\
7311.7
\end{tabular} & $\begin{array}{l}3474.4 \\
3491.3 \\
35082 \\
3525.2 \\
3542.4 \\
3559.6\end{array}$ & $\begin{array}{c}10^{\circ} \mathrm{C} . \\
T \\
.103 \\
E \\
.536\end{array}$ & $\begin{array}{r}113^{\circ} \\
10^{\prime} \\
20^{\prime} \\
30^{\prime} \\
40^{\prime} \\
50^{\prime}\end{array}$ & $\begin{array}{l}8656.6 \\
8684.0 \\
8711.5 \\
8739.2 \\
8767.0 \\
8794.9\end{array}$ & $\begin{array}{l}46513 \\
4674.2 \\
4697.2 \\
4720.3 \\
4743.6 \\
4766.9\end{array}$ & $\begin{array}{c}10 . \mathrm{C} . \\
T \\
1.25 \\
E \\
.721\end{array}$ \\
\hline $\begin{array}{r}94^{\circ} \\
10^{\prime} \\
20^{\prime} \\
30^{\prime} \\
40^{\prime} \\
50^{\prime}\end{array}$ & $\begin{array}{l}6144.3 \\
6162.2 \\
6180.2 \\
6198.3 \\
6216.4 \\
6234.6\end{array}$ & $\left|\begin{array}{l}2671.6 \\
2684.7 \\
2697.9 \\
2711.2 \\
2724.5 \\
2737.9\end{array}\right|$ & $15^{\circ} \mathrm{C}$ & $\begin{array}{r}104^{\circ} \\
10^{\prime} \\
20^{\prime} \\
30 \\
40^{\prime} \\
50^{\circ}\end{array}$ & $\begin{array}{l}7333.6 \\
7355.6 \\
7377.8 \\
7399.9 \\
7422.2 \\
7444.6\end{array}$ & $\begin{array}{l}3576.8 \\
3594.2 \\
3611.7 \\
3629.2 \\
3646.8 \\
3664.5\end{array}$ & $15^{\circ} \mathrm{C}$. & $\begin{array}{r}114^{\circ} \\
10^{\prime} \\
20^{\prime} \\
30^{\prime} \\
40^{\prime} \\
50^{\prime}\end{array}$ & $\begin{array}{l}8822.9 \\
8851.0 \\
8879.3 \\
8907.7 \\
8936.3 \\
8965.0\end{array}$ & $\begin{array}{l}4790.4 \\
4814.1 \\
4837.8 \\
4861.7 \\
4885.7 \\
4909.9\end{array}$ & $15^{\circ}$ \\
\hline $\begin{array}{r}95^{\circ} \\
10^{\prime} \\
20^{\prime} \\
30^{\prime} \\
40^{\prime} \\
50^{\prime}\end{array}$ & $\begin{array}{l}6252.8 \\
6271.1 \\
6289.4 \\
6307.9 \\
6326.3 \\
6344.8\end{array}$ & \begin{tabular}{|l|}
2751.3 \\
2764.8 \\
2778.3 \\
2792.0 \\
2805.6 \\
2819.4
\end{tabular} & $\begin{array}{c}T \\
1.30 \\
E \\
.604\end{array}$ & $\begin{array}{r}105^{\circ} \\
10^{\prime} \\
20^{\circ} \\
30^{\circ} \\
40^{\prime} \\
50^{\circ}\end{array}$ & $\begin{array}{l}7467.0 \\
7489.6 \\
7512.2 \\
7534.9 \\
7557.7 \\
7580.5\end{array}$ & $\begin{array}{l}3682.3 \\
3700.2 \\
3718.2 \\
3736.2 \\
3754.4 \\
3772.6\end{array}$ & $\begin{array}{c}T \\
1.56 \\
E \\
.806\end{array}$ & $\begin{array}{r}115^{\circ} \\
10^{\prime} \\
20^{\prime} \\
30^{\prime} \\
40^{\prime} \\
50^{\prime}\end{array}$ & $\begin{array}{l}8993.8 \\
9022.7 \\
9051.7 \\
9080.9 \\
9110.3 \\
9139.8\end{array}$ & $\begin{array}{l}4934.1 \\
4958.6 \\
4983.1 \\
5007.8 \\
5032.6 \\
5057.6\end{array}$ & $\begin{array}{c}T \\
1.93 \\
E \\
1.09\end{array}$ \\
\hline $\begin{array}{r}96^{\circ} \\
10 \\
20^{\prime} \\
30^{\prime} \\
40^{\prime} \\
50^{\prime}\end{array}$ & $\begin{array}{l}6363.4 \\
6382.1 \\
6400.8 \\
6419.5 \\
6438.4 \\
6457.3\end{array}$ & $\begin{array}{l}2833.2 \\
2847.0 \\
2861.0 \\
2875.0 \\
2889.0 \\
2903.1\end{array}$ & $\begin{array}{c}20^{\circ} \mathrm{C} \\
\mathrm{T} \\
1.74\end{array}$ & $\begin{array}{r}106^{\circ} \\
10^{\prime} \\
20^{\circ} \\
30^{\prime} \\
40^{\prime} \\
50^{\prime}\end{array}$ & \begin{tabular}{|l|}
7603.5 \\
7626.6 \\
7649.7 \\
7672.9 \\
7696.3 \\
7719.7
\end{tabular} & $\begin{array}{l}3791.0 \\
3809.4 \\
3827.9 \\
3846.5 \\
3865.2 \\
3884.0\end{array}$ & $\begin{array}{c}20^{\circ} \mathrm{C} . \\
\mathrm{T} \\
2.08\end{array}$ & $\begin{array}{c}16^{\circ} \\
10^{\prime} \\
20^{\prime} \\
30^{\prime} \\
40^{\prime} \\
50^{\prime}\end{array}$ & \begin{tabular}{|}
9169.4 \\
9199.1 \\
9229.0 \\
9259.0 \\
9289.2 \\
9319.5
\end{tabular} & $\begin{array}{l}5082.7 \\
5107.9 \\
5133.3 \\
5158.8 \\
5184.5 \\
5210.3\end{array}$ & $\begin{array}{c}20^{\circ} \mathrm{C} . \\
T\end{array}$ \\
\hline $\begin{array}{r}97 \\
10^{\circ} \\
20^{\prime} \\
30^{\prime} \\
40^{\prime} \\
50^{\prime}\end{array}$ & $\begin{array}{l}6476.2 \\
6495.2 \\
6514.3 \\
6533.4 \\
6552.6 \\
6571.9\end{array}$ & $\begin{array}{l}2917.3 \\
2931.6 \\
2945.9 \\
2960.3 \\
2974.7 \\
2989.2\end{array}$ & $\begin{array}{c}E \\
.809\end{array}$ & $\begin{array}{r}107^{\circ} \\
10^{\prime} \\
20^{\prime} \\
30^{\prime} \\
40^{\prime} \\
50^{\prime}\end{array}$ & \begin{tabular}{|l|}
7743.2 \\
7766.8 \\
7790.5 \\
7814.3 \\
7838.1 \\
7862.1
\end{tabular} & $\begin{array}{l}3902.9 \\
3921.9 \\
3940.9 \\
3960.1 \\
3979.4 \\
3998.7\end{array}$ & $\begin{array}{l}\text { E } \\
1.08\end{array}$ & $\mid \begin{array}{r}117^{\circ} \\
10^{\prime} \\
20^{\prime} \\
30^{\prime} \\
40^{\prime} \\
50^{\prime}\end{array}$ & $\begin{array}{l}9349.9 \\
9380.5 \\
9411.3 \\
9442.2 \\
9473.2 \\
9504.4\end{array}$ & $\begin{array}{l}5236.2 \\
5262.3 \\
5288.6 \\
5315.0 \\
5341.5 \\
5368.2\end{array}$ & $\begin{array}{c}2.52 \\
E \\
1.46\end{array}$ \\
\hline $\begin{array}{r}98^{\circ} \\
10^{\prime} \\
20^{\prime} \\
30^{\prime} \\
40^{\prime} \\
50^{\prime}\end{array}$ & $\begin{array}{l}6591.2 \\
6610.6 \\
6630.1 \\
6649.6 \\
6669.2 \\
6688.8\end{array}$ & \begin{tabular}{|l|}
3003.8 \\
3018.4 \\
3033.1 \\
3047.9 \\
3062.8 \\
3077.7
\end{tabular} & $\begin{array}{c}25^{\circ} \mathrm{C} \\
\mathrm{T} \\
2.18 \\
\mathrm{E}\end{array}$ & $\mid \begin{array}{r}108 \\
10 \\
20 \\
30^{\circ} \\
40^{\circ} \\
50^{\prime}\end{array}$ & \begin{tabular}{|l|}
7886.2 \\
7910.4 \\
7934.6 \\
$7959 . C$ \\
7983.5 \\
8008.0
\end{tabular} & $\begin{array}{l}4018.2 \\
4037.8 \\
4057.4 \\
4077.2 \\
4097.1 \\
4117.0\end{array}$ & $\begin{array}{c}25=C \\
T \\
2.61 \\
E\end{array}$ & $\begin{array}{r}18^{\circ} \\
10^{\prime} \\
20^{\circ} \\
30^{\prime} \\
40^{\prime} \\
50^{\prime}\end{array}$ & $\begin{array}{l}9535.7 \\
9567.2 \\
9598.9 \\
9630.7 \\
9662.6 \\
9694.7\end{array}$ & $\begin{array}{l}5395.1 \\
5422.1 \\
5449.2 \\
5476.5 \\
5504.0 \\
5531.7\end{array}$ & $\begin{array}{c}25^{\circ} \mathrm{C} . \\
\mathrm{T} \\
3.16 \\
\mathrm{E}\end{array}$ \\
\hline $\begin{array}{l}99^{\circ} \\
10^{\prime} \\
20^{\prime}\end{array}$ & $\begin{array}{l}6708.6 \\
6728.4 \\
6748.2\end{array}$ & $\begin{array}{l}3092.7 \\
3107.7 \\
3122.9\end{array}$ & 1.02 & $\begin{array}{r}109^{\circ} \\
10^{\prime} \\
20^{\prime}\end{array}$ & $\begin{array}{l}8032.7 \\
8057.4 \\
8082.3\end{array}$ & $\begin{array}{l}4137,1 \\
4157,3 \\
4177.5\end{array}$ & 1.36 & $\begin{array}{r}119^{\circ} \\
10^{\circ} \\
20^{\prime}\end{array}$ & $\begin{array}{l}9727.0 \\
9759.4 \\
9792.0\end{array}$ & $\begin{array}{l}5559.4 \\
5587.4 \\
5615.5\end{array}$ & 1.83 \\
\hline $\begin{array}{l}30^{\prime} \\
40^{\prime} \\
50^{\prime}\end{array}$ & $\begin{array}{l}6768.1 \\
6788.1 \\
6808.2\end{array}$ & $\begin{array}{l}3138.1 \\
3153.3 \\
3168.7\end{array} \mid$ & & $\begin{array}{l}30^{\prime} \\
40^{\prime} \\
50^{\prime}\end{array}$ & $\begin{array}{l}8107.3 \\
8132.3 \\
8157.5\end{array}$ & $\begin{array}{l}4197.9 \\
4218.4 \\
4239.0\end{array}$ & & $\begin{array}{l}30 \\
40^{\prime} \\
50^{\prime}\end{array}$ & $\begin{array}{l}9824.8 \\
9857.7 \\
9890.8\end{array}$ & $\begin{array}{l}5643.8 \\
5672.3 \\
5700.9\end{array}$ & \\
\hline $\begin{array}{r}100^{\circ} \\
10^{\prime} \\
20^{\prime} \\
30^{\prime} \\
40^{\circ} \\
50^{\circ}\end{array}$ & $\begin{array}{l}6828.3 \\
6848.5 \\
6868.8 \\
6889.2 \\
6909.6 \\
6930.1\end{array}$ & $\begin{array}{l}3184.1 \\
3199.6 \\
3215.1 \\
3230.8 \\
3246.5 \\
3262.3\end{array}$ & $\begin{array}{c}30^{\circ} \mathrm{C} \\
\mathrm{T} \\
2.62 \\
\mathrm{E} \\
1.22\end{array}$ & $\begin{array}{r}110^{\circ} \\
10 \\
20^{\prime} \\
30^{\circ} \\
40^{\prime} \\
50^{\prime}\end{array}$ & \begin{tabular}{|l|}
8182.8 \\
8208.2 \\
8233.7 \\
8259.3 \\
8285.0 \\
8310.8
\end{tabular} & $\begin{array}{l}4259.7 \\
4280.5 \\
4301.4 \\
4322.4 \\
4343.6 \\
4364.8\end{array}$ & $\begin{array}{c}30{ }^{\circ} \mathrm{C} . \\
T \\
3.14 \\
\mathrm{E} \\
1.63\end{array}$ & $\begin{array}{r}120^{\circ} \\
10^{\circ} \\
20^{\prime} \\
30^{\prime} \\
40^{\prime} \\
50^{\prime}\end{array}$ & $\begin{array}{r}9924.0 \\
9957.5 \\
9991.0 \\
10025.0 \\
10059.0 \\
10093.0\end{array}$ & $\begin{array}{l}5729.7 \\
5758.6 \\
5787.7 \\
5817.0 \\
5846.5 \\
5876.1\end{array}$ & $\begin{array}{c}30 \text { C. } \\
T \\
3.81 \\
E \\
2.20\end{array}$ \\
\hline
\end{tabular}




\section{USEFUL RELATIONS}

\begin{tabular}{|c|c|c|}
\hline Lineal feet & $\times .00019$ & $=$ miles \\
\hline Lineal yards & $\times .0006$ & $=$ miles \\
\hline Square inches & $\times .007$ & $=$ square feet \\
\hline Square feet & $\times .111$ & $=$ square yards \\
\hline Square yards & $\times .0002067$ & $=$ acres \\
\hline Acres & $\times 4840$ & $=$ square yards \\
\hline Cubic inches & $\times .00058$ & $=$ cubic feet \\
\hline Cubic feet & $\times .03704$ & $=$ cubic yards \\
\hline Links & $\times .22$ & $=$ yards \\
\hline Links & $\times .66$ & $=$ feet \\
\hline Feet & $\times 1.5$ & $=$ links \\
\hline \multicolumn{3}{|c|}{$360^{\circ}=21600^{\circ}=1296000^{\prime \prime}$} \\
\hline \multicolumn{3}{|c|}{ Radius $=$ arc of $57.2957790^{\circ}$} \\
\hline \multicolumn{3}{|c|}{ Arc of $1^{\circ}($ radius $=1)=.017453292$} \\
\hline \multicolumn{3}{|c|}{ Arc of $1^{\prime}($ radius $=1)=.000290888$} \\
\hline Arc of $1^{\prime \prime}$ (radi & us $=1)=$ & .000004848 \\
\hline
\end{tabular}

Curvature of Earth's surface $=$ about 0.7 feet in 1 mile Curvature in feet $=0.667$ (Dist. in miles) ${ }^{2}$

Difference between arc and chord length, 0.05 feet in $111 / 2$ miles

Probable error of a single observation $=0.6754 \sqrt{\frac{\sum v^{2}}{n-1}}$

Error in chaining of 0.01 feet in 100 feet:

Due to-

1. Length of tape error of 0.01 feet

2. Alignment. One end 1.4 feet out of line

3. Sag of tape at center of 0.61 feet.

4. Temperature difference of $15^{\circ}$

5. Difference of pull of $\mathbf{1 5} \mathrm{lbs}$.

SQUARE MEASURE

144 sq. inches $=1$ sq. $\mathrm{ft}$.

9 sq. ft. $=1$ sq. yard

$30 \frac{1}{4}$ sq. yds. $=1$ sq. rd.

40 sq. rds. $=1$ rood.

4 roods $=1$ acre

640 acres $=1$ sq. mile.

SURVEYORS' MEASURE

7.92 inches $=1$ link.

25 links $=1$ red.

4 rds. = 1 chain.

10 sq. chains or 160 sq. rods $=1$ acre.

640 acres $=1$ sq. mile.

36 sq. miles ( 6 miles sq.) $=1$ township. 
TABLE VI. INCHES TO DECIMALS OF A FOOT

\begin{tabular}{|c|c|c|c|c|c|c|c|c|c|c|c|c|c|}
\hline In. & 0 & 1 & 2 & 3 & 4 & 5 & 6 & 7 & 8 & 9 & 10 & 11 & n. \\
\hline $\begin{array}{l}0 \\
1-32 \\
1-16 \\
3-32 \\
1-8 \\
5-32 \\
3-16 \\
7-32\end{array}$ & $\begin{array}{l}\text { Foot } \\
.0026 \\
.0052 \\
.0078 \\
.0104 \\
.0130 \\
0156 \\
.0189 \\
.0189\end{array}$ & $\begin{array}{l}.0833 \\
.08599 \\
.0885 \\
.0911 \\
.0938 \\
.096464 \\
.04990 \\
.1016\end{array}$ & $\begin{array}{l}.1667 \\
.1693 \\
.1719 \\
.1745 \\
.1771 \\
.1797 \\
.18 \% 3 \\
.1849\end{array}$ & $\begin{array}{l}.2500 \\
.2526 \\
.2552 \\
.25 \pi 8 \\
.2604 \\
.2630 \\
.2605 \\
.2682\end{array}$ & $\begin{array}{l}.3333 \\
.33159 \\
.3355 \\
.3411 \\
3438 \\
3464 \\
.3490 \\
.3516\end{array}$ & $\begin{array}{r}4167 \\
.4193 \\
.4219 \\
.4245 \\
.4271 \\
.4297 \\
4323 \\
4349\end{array}$ & $\begin{array}{l}.5000 \\
.5026 \\
.5052 \\
.5078 \\
.5104 \\
.5130 \\
.5156 \\
.5182\end{array}$ & $\begin{array}{l}5833 \\
.5859 \\
58885 \\
.5411 \\
59338 \\
59414 \\
5900 \\
6016\end{array}$ & $\begin{array}{l}.6667 \\
.0693 \\
.6719 \\
.6745 \\
.6771 \\
.6797 \\
.68323 \\
.6849\end{array}$ & $\begin{array}{l}.7500 \\
.7526 \\
.7552 \\
.7578 \\
.7604 \\
.76030 \\
.7656 \\
.7682\end{array}$ & $\begin{array}{l}.8333 \\
.8359 \\
.8385 \\
.8411 \\
84138 \\
8464 \\
8490 \\
8516\end{array}$ & $\begin{array}{l}.9167 \\
.9193 \\
.9219 \\
.9245 \\
.9271 \\
.9297 \\
.9329 \\
.9349\end{array}$ & $\begin{array}{l}0 \\
1-32 \\
1-16 \\
2-32 \\
1-8 \\
5-32 \\
3-16 \\
7-32\end{array}$ \\
\hline $\begin{array}{r}1-4 \\
9-32 \\
5-16 \\
11-32 \\
3-8 \\
13-22 \\
7-16 \\
15-32\end{array}$ & $\begin{array}{r}.0208 \\
.0234 \\
.02860 \\
.0286 \\
.0313 \\
.0339 \\
.0365 \\
.0391\end{array}$ & $\begin{array}{r}.1042 \\
.1068 \\
.1094 \\
.1129 \\
.1146 \\
.1172 \\
.1198 \\
.1224\end{array}$ & $\begin{array}{l}.1875 \\
.1901 \\
.1927 \\
.1953 \\
.1979 \\
.2005 \\
.2031 \\
.2057\end{array}$ & $\begin{array}{l}.2708 \\
.2734 \\
.2760 \\
2780 \\
.2813 \\
.2839 \\
.2865 \\
.2891\end{array}$ & $\begin{array}{r}3542 \\
3568 \\
3594 \\
3620 \\
3646 \\
3672 \\
.3698 \\
.3724\end{array}$ & $\begin{array}{l}.4375 \\
.4401 \\
.4427 \\
.4453 \\
.4479 \\
.4505 \\
.4531 \\
.4557\end{array}$ & $\begin{array}{l}.5308 \\
.5234 \\
.5260 \\
.5236 \\
.53313 \\
.53399 \\
.53565 \\
.5391\end{array}$ & $\begin{array}{l}.6042 \\
.6068 \\
6094 \\
6120 \\
.6146 \\
.6172 \\
.6198 \\
.6224\end{array}$ & $\begin{array}{l}.9875 \\
6901 \\
6927 \\
6953 \\
6979 \\
.7005 \\
7031 \\
.7057\end{array}$ & $\begin{array}{l}T 08 \\
7.44 \\
760 \\
786 \\
7813 \\
.7865 \\
.7 \% 31\end{array}$ & $\begin{array}{l}8542 \\
.8568 \\
8594 \\
8620 \\
.8646 \\
.8672 \\
.8698 \\
.8 \pi 24\end{array}$ & $\begin{array}{l}.9375 \\
.9401 \\
.9427 \\
.9453 \\
.9479 \\
.9505 \\
.9531 \\
.9557\end{array}$ & $\begin{array}{c}1-4 \\
9-32 \\
6-16 \\
11-32 \\
3-8 \\
13-32 \\
7-16 \\
15-32\end{array}$ \\
\hline $\begin{array}{c}1-2 \\
17-32 \\
9-16 \\
19-32 \\
5-8 \\
21-32 \\
11-16 \\
23-32\end{array}$ & $\begin{array}{l}.0417 \\
.0443 \\
.0468 \\
.0495 \\
.0521 \\
.054 \\
.0553 \\
.0590\end{array}$ & $\begin{array}{l}1250 \\
1276 \\
1302 \\
.1328 \\
.1354 \\
.1350 \\
.1406 \\
.1132\end{array}$ & $\begin{array}{l}2083 \\
2109 \\
.2135 \\
2161 \\
2189 \\
2214 \\
.2210 \\
.2260\end{array}$ & $\begin{array}{l}.2917 \\
2+13 \\
.2069 \\
.2095 \\
.3021 \\
.3047 \\
.306 \\
.3000\end{array}$ & $\begin{array}{l}.3750 \\
3776 \\
3809 \\
.38253 \\
.3851 \\
.3890 \\
.3906 \\
.3932\end{array}$ & $\begin{array}{r}.4583 \\
.4609 \\
4695 \\
.4661 \\
4688 \\
.4714 \\
4740 \\
4766\end{array}$ & $\begin{array}{l}.5417 \\
.5443 \\
.5469 \\
.5495 \\
.5521 \\
.5547 \\
.5573 \\
.5599\end{array}$ & $\begin{array}{l}.6250 \\
.6276 \\
6302 \\
63030 \\
6354 \\
.6380 \\
6406 \\
.6430\end{array}$ & $\begin{array}{l}.7083 \\
.7109 \\
.7135 \\
.7161 \\
.7188 \\
.7214 \\
7240 \\
.7960\end{array}$ & $\begin{array}{l}.7917 \\
.7943 \\
.7009 \\
.7905 \\
8021 \\
.8047 \\
.8073 \\
.8099\end{array}$ & $\begin{array}{l}.8750 \\
.8766 \\
.8802 \\
.8858 \\
.8554 \\
.8880 \\
8906 \\
.8932\end{array}$ & $\begin{array}{l}.0583 \\
.9609 \\
.9635 \\
.9661 \\
.9688 \\
.9714 \\
9740 \\
.9766\end{array}$ & $\begin{array}{c}1-2 \\
17-32 \\
9-16 \\
19-32 \\
5-8 \\
21-32 \\
11-10 \\
20-32\end{array}$ \\
\hline $\begin{array}{c}3-4 \\
25-32 \\
13-16 \\
27-32 \\
7-8 \\
29-33 \\
15-16 \\
31-32\end{array}$ & $\begin{array}{l}.071 \\
.0703 \\
.0728 \\
.0755 \\
.0781 \\
.0807\end{array}$ & $\begin{array}{l}1510 \\
.1536 \\
.1563 \\
.1589 \\
.1615 \\
.1641\end{array}$ & $\begin{array}{l}2292 \\
2316 \\
244 \\
2370 \\
.2496 \\
.2422 \\
.2448 \\
.2474\end{array}$ & 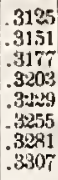 & $\begin{array}{r}.3958 \\
.3944 \\
.4010 \\
.40366 \\
.4063 \\
.4089 \\
.4115 \\
.4141\end{array}$ & $\begin{array}{l}4732 \\
.4818 \\
.46+4 \\
4870 \\
.4896 \\
4922 \\
.4948 \\
.4974\end{array}$ & $\begin{array}{l}.5625 \\
.5651 \\
.5677 \\
.5703 \\
.5729 \\
.5755 \\
.5781 \\
.5807\end{array}$ & $\begin{array}{l}6589 \\
6615 \\
.6641\end{array}$ & $\begin{array}{r}7292 \\
-7315 \\
7344 \\
7370 \\
.7396 \\
.7424 \\
7448 \\
.7474\end{array}$ & $\begin{array}{l}.8125 \\
.8151 \\
.817 \\
.8203 \\
.8229 \\
.8225 \\
.8281 \\
8307\end{array}$ & $\begin{array}{l}.8958 \\
.8934 \\
.9010 \\
.9036 \\
.9063 \\
.9089 \\
.9115 \\
.9141\end{array}$ & $\begin{array}{l}.9896 \\
.9922 \\
.9948 \\
.9974\end{array}$ & $\begin{array}{c}3-4 \\
25-32 \\
13-16 \\
27-32 \\
7-8 \\
29-32 \\
15-16 \\
31-39\end{array}$ \\
\hline & 0 & 1 & 2 & 3 & 4 & 5 & 6 & 7 & 8 & 9 & 10 & 11 & \\
\hline
\end{tabular}

TABLE VII. MINUTES IN DECIMALS OF A DEGREE

\begin{tabular}{|c|c|c|c|c|c|c|c|c|c|c|c|}
\hline $0^{\prime} 30^{\prime \prime}$ & 0833 & 30 & .17500 & $20^{\prime} 30^{\prime \prime}$ & .34167 & $30^{\prime} 30^{\prime \prime}$ & .50833 & $40^{\prime} 30^{\prime \prime}$ & .67500 & $50^{\prime} 30^{\prime}$ & .84167 \\
\hline 100 & .01667 & $\begin{array}{ll}11 & 00\end{array}$ & .18333 & 2100 & .35000 & $\begin{array}{ll}31 & 00\end{array}$ & .51667 & $\begin{array}{ll}41 & 00\end{array}$ & .68333 & $\begin{array}{ll}51 & 00\end{array}$ & .85000 \\
\hline 30 & .02500 & 30 & .19167 & 30 & .35833 & 30 & .52500 & 30 & .69167 & 30 & . 85833 \\
\hline 200 & .03333 & 1200 & .20000 & 2200 & .36667 & 3200 & .53333 & $\begin{array}{lll}42 & 00\end{array}$ & .70000 & $\begin{array}{lll}52 & 00\end{array}$ & .86667 \\
\hline 30 & .04167 & 30 & .20833 & 30 & .37500 & 30 & .54167 & 30 & .70833 & 30 & .87500 \\
\hline 300 & .050000 & 1300 & .21667 & 2300 & .38333 & 3300 & .55000 & $\begin{array}{lll}43 & 00\end{array}$ & .71667 & $\begin{array}{lll}53 & 00\end{array}$ & .88333 \\
\hline 30 & .05833 & 30 & .22500 & 30 & .39167 & 30 & .55833 & 30 & .72500 & 30 & 89167 \\
\hline 400 & .06667 & 1400 & .23333 & 2400 & .40000 & 3400 & .56667 & 4400 & .73333 & 5400 & .90000 \\
\hline 30 & .07500 & 30 & .24167 & 30 & .40833 & 30 & .57500 & 30 & .74167 & 30 & (90833. \\
\hline 500 & .08333 & $15 \mathrm{~m}$ & .25000 & 2500 & .41667 & $\begin{array}{ll}35 & 00\end{array}$ & .58333 & 4500 & .75000 & $\begin{array}{lll}55 & 00\end{array}$ & .91667 \\
\hline 30 & .09167 & 30 & 25833 & 30 & .42500 & 30 & .59167 & 30 & .75833 & 30 & .92500 \\
\hline 600 & .10000 & 1600 & .26667 & 2600 & .43333 & $\begin{array}{ll}36 & 00\end{array}$ & .60000 & 4600 & .76667 & $\begin{array}{lll}56 & 00^{\circ}\end{array}$ & .93333 \\
\hline 30 & 33 & 30 & .27500 & 30 & & 30 & 33 & 30 & 00 & 30 & .94167 \\
\hline 700 & .11667 & 1700 & 28333 & 2700 & .45000 & 3700 & .61667 & 4700 & .78333 & 5700 & .95000 \\
\hline 30 & .12500 & 30 & .29167 & 30 & .45833 & 30 & .62500 & 30 & .79167 & 30 & .95833 \\
\hline 800 & .13333 & 1800 & .30000 & 2800 & .46667 & 3800 & .63333 & $\begin{array}{lll}48 & 00\end{array}$ & .80 & 800 & .96667 \\
\hline 30 & .14167 & 30 & .30833 & 30 & .47500 & 30 & .64167 & 30 & .80 & 30 & .97500 \\
\hline 00 & .15000 & 1900 & .31667 & \begin{tabular}{|ll}
29 & 00
\end{tabular} & .48333 & 3900 & .65000 & 4900 & .81667 & $\mid$\begin{tabular}{|lll}
59 & 00
\end{tabular} & .98333 \\
\hline 30 & .15833 & & .32500 & 30 & .49167 & 30 & .65833 & 30 & & 30 & \\
\hline $10 \quad 00$ & .16667 & $\begin{array}{ll}20 & 00\end{array}$ & .33333 & $\begin{array}{ll}30 & 00\end{array}$ & .500000 & $\begin{array}{lll}40 & 00\end{array}$ & .66667 & $\begin{array}{ll}50 & 00\end{array}$ & .83333 & $\begin{array}{lll}60 & 00\end{array}$ & 1.00000 \\
\hline
\end{tabular}


TABI.E VIII. MIDDLE ORDINATES OF RAILS

Length of Rail (feet)

\begin{tabular}{|c|c|c|c|c|c|c|c|c|c|c|c|c|c|c|c|}
\hline $\begin{array}{l}\mathrm{C} \\
0 \quad 1\end{array}$ & $\begin{array}{c}\mathrm{R} \\
\text { Feet } \\
\end{array}$ & $\begin{array}{c}30 \\
\text { Inch }\end{array}$ & \begin{tabular}{|l|}
28 \\
Inch \\
\end{tabular} & $\begin{array}{l}26 \\
\text { Ioch }\end{array}$ & $\begin{array}{c}24 \\
\text { Inch } \\
\end{array}$ & $\begin{array}{l}22 \\
\text { lnch }\end{array}$ & $\begin{array}{l}20 \\
\text { Inch }\end{array}$ & $\begin{array}{l}\mathrm{C} \\
\mathrm{O}\end{array}$ & $\begin{array}{r}\mathbf{R} \\
\text { Fett }\end{array}$ & \begin{tabular}{|l}
30 \\
Inch \\
\end{tabular} & \begin{tabular}{|c|}
28 \\
lack \\
\end{tabular} & \begin{tabular}{|l|}
26 \\
Inch \\
\end{tabular} & \begin{tabular}{|l|}
24 \\
Inch \\
\end{tabular} & $\begin{array}{l}22 \\
\text { Ioch } \\
\end{array}$ & $\begin{array}{l}20 \\
\text { Inch }\end{array}$ \\
\hline-20 & 17189 & .08 & .07 & .06 & .05 & .04 & .03 & 8 & 716.8 & 88 & 1,64 & 4! & .2 & 1.01 & .84 \\
\hline-40 & 8594 & .16 & .14 & .12 & .10 & .08 & .07 & 9 & 637.3 & 12 & 1.8 & 1.60 & 1.36 & 14 & .94 \\
\hline-0 & 5730 & .24 & .20 & .18 & .15 & .13 & .10 & 10 & 573.7 & 36 & 2.05 & 1.78 & 1.50 & 1.27 & 1.04 \\
\hline-20 & 4297 & .31 & .27 & .23 & .20 & .17 & .13 & 11 & 521.7 & 6 & 2.26 & 1.95 & 6 & 1.38 & 1.15 \\
\hline $1-40$ & 3438 & .39 & .34 & .29 & .25 & 21 & .17 & 12 & 478.3 & 3.83 & 2.47 & 2.15 & 1.81 & 1.5 & 1.26 \\
\hline $2-0$ & 2865 & .47 & .41 & .35 & .30 & .25 & .20 & 13 & 441.7 & 3.05 & 2.66 & $|2.30|$ & 1.96 & 1.66 & 1.36 \\
\hline $2-20$ & 2456 & .55 & .48 & .41 & .35 & .29 & .23 & 14 & 410.3 & 3.30 & 2.87 & 2.48 & 2.10 & 1.78 & 1.46 \\
\hline $2-40$ & 2149 & .63 & .55 & .47 & .40 & .33 & .27 & 15 & 383.1 & 3.5 & 3.08 & 2.68 & 2.26 & 1.91 & 1.67 \\
\hline $3-0$ & 1910 & .71 & .62 & .53 & .45 & .38 & .31 & 16 & 359.3 & 3.76 & 3.28 & 2.83 & 2.40 & 2.0 & 1.67 \\
\hline $3-20$ & 1719 & .78 & .68 & .59 & .50 & .42 & .35 & 17 & 338.3 & 4.00 & 3.48 & 3.02 & 2.57 & 2.16 & 1.78 \\
\hline $3-40$ & 1563 & .86 & 75 & .65 & .55 & .46 & .38 & 18 & 319.6 & 4.21 & 3.67 & 3.18 & 2.70 & 228 & 1.87 \\
\hline 4 & 1433 & .94 & .82 & .71 & .60 & .50 & .42 & 18 & 302.9 & 4.45 & 3.8 & $\mid 3.36$ & 2.80 & 2.41 & 1.98 \\
\hline 4 & 1323 & 1.02 & .89 & .77 & .65 & .65 & .45 & 20 & 287.9 & 4.70 & 4.00 & 3.55 & 3.00 & 2.54 & 2.09 \\
\hline 4 & 1228 & 1.10 & .96 & .83 & .70 & .59 & .48 & 22 & 262.0 & 5.16 & 4.44 & 3.84 & 3.30 & 2.80 & 2.29 \\
\hline 5 & 1146 & 1.18 & 1.03 & .88 & .75 & .63 & .52 & 24 & 240.5 & 5.64 & 4.92 & 4.20 & 3.59 & 3.04 & 2.50 \\
\hline 6 & 955.3 & 1.41 & 1.23 & 1.06 & .90 & .76 & .62 & 26 & 222.3 & 6.07 & 5.29 & 4.58 & 3.8 & 3.29 & 2.70 \\
\hline & 819.0 & 1.6 & .44 & & 05 & .89 & .73 & & & & & & & & \\
\hline
\end{tabular}

TABLE IX. SHORT RADIUS CURVES

\begin{tabular}{||c|c|c|c|c|c||}
\hline \hline & $\begin{array}{c}\text { Redius } \\
\text { Foet }\end{array}$ & $\begin{array}{c}\text { Obord } \\
\text { Feet }\end{array}$ & $\begin{array}{c}\text { Contral } \\
\text { Angle }\end{array}$ & $\begin{array}{c}\text { Defloction } \\
\text { Angle }\end{array}$ & $\begin{array}{c}\text { Doflootion } \\
\text { for 1 Foot }\end{array}$ \\
\hline 35 & 10 & $16-26$ & $8-13$ & 49.3 \\
45 & 10 & $12-46$ & $6-23$ & 38.3 \\
50 & 15 & $17-16$ & $8-38$ & 34.5 \\
60 & 15 & $14-22$ & $7-11$ & 28.8 \\
75 & 15 & $11-30$ & $5-45$ & 23.0 \\
100 & 20 & $11-30$ & $5-45$ & 17.3 \\
120 & 20 & $9-34$ & $4-47$ & 14.3 \\
150 & 20 & $7-39$ & $3-49$ & 11.5 \\
190 & 25 & $7-32$ & $3-46$ & 9.15 \\
200 & 25 & $7-10$ & $3-35$ & 8.6 \\
225 & 25 & $6-25$ & $3-12$ & 7.7 \\
& 240 & 25 & $5-58$ & $2-59$ & 7.2 \\
& 250 & 25 & $5-44$ & $2-52$ & 6.9 \\
& 275 & 25 & $5-12$ & $2-36$ & 6.2 \\
& 288 & 50 & $9-58$ & $4-59$ & 6.0 \\
& 300 & 50 & $9-32$ & $4-46$ & 5.7 \\
& 350 & 50 & $8-12$ & $4-06$ & 4.9 \\
& 376 & 50 & $7-40$ & $3-50$ & 4.6 \\
& 400 & 50 & $7-10$ & $3-35$ & 4.3 \\
& 410 & 50 & $7-00$ & $3-30$ & 4.2 \\
\hline
\end{tabular}

To find length of curve divide angle from P. C. to P. T. by central angle of chord, and multiply by length of chord. 
TABLE X. RODS IN FEET, 10THS AND 100TIIS OF FEET

\begin{tabular}{|c|c|c|c|c|c|c|c|c|c|}
\hline $\begin{array}{c}\stackrel{n}{8} \\
\dot{0} \\
\end{array}$ & Feet & 莒 & Feet & $\begin{array}{l}\text { 量 } \\
\text { 足 }\end{array}$ & Feet & $\begin{array}{l}\bar{q} \\
\stackrel{0}{0} \\
\tilde{n}\end{array}$ & Feet & वृ & Feet \\
\hline$\overline{1}$ & 16.50 & 21 & 346.50 & 41 & 676.50 & 61 & 1006.50 & 81 & 1336.50 \\
\hline 2 & 33.00 & 22 & 363.00 & 42 & 693.00 & 62 & 1023.00 & 82 & 1353.00 \\
\hline 3 & 49.50 & 23 & 379.50 & 43 & 709.50 & 63 & 1039.50 & 83 & 1369.50 \\
\hline 4 & 66.00 & 24 & 396.00 & 44 & 726.00 & 64 & 1056.00 & 84 & 1386.00 \\
\hline 5 & 82.50 & 25 & 412.50 & 45 & 742.50 & 65 & 1072.50 & 85 & 1402.50 \\
\hline 6 & 99.00 & 26 & 429.00 & 46 & 759.00 & 66 & 1089.00 & 86 & 1419.00 \\
\hline 7 & 115.50 & 27 & 445.50 & 47 & 775.50 & 67 & 1105.50 & 87 & 1435.50 \\
\hline 8 & 132.00 & 28 & 462.00 & 48 & 792.00 & 68 & 1122.00 & 88 & 1452.00 \\
\hline 9 & 148.50 & 29 & 478.50 & 49 & 808.50 & 69 & 1138.50 & 89 & 1468.50 \\
\hline 10 & 165.00 & 30 & 495.00 & 50 & 825.00 & 70 & 1155.00 & 90 & 1485.00 \\
\hline 11 & 181.50 & 31 & 511.50 & 51 & 841.50 & 71 & 1171.50 & 91 & 1501.50 \\
\hline 12 & 198.00 & 32 & 528.00 & 52 & 858.00 & 72 & 1188.00 & 92 & 1518.00 \\
\hline 13 & 214.50 & 33 & 544.50 & 53 & 874.50 & 73 & 1204.50 & 93 & 1534.50 \\
\hline 14 & 231.00 & 34 & 561.00 & 54 & 891.00 & 74 & 1221.00 & 94 & 1551.00 \\
\hline 15 & 247.50 & 35 & 577.50 & 55 & 907.50 & 75 & 1237.50 & 95 & 1567.50 \\
\hline 16 & 264.00 & 36 & 594.00 & 56 & 924.00 & 76 & 1254.00 & 96 & 1584.00 \\
\hline 17 & 280.50 & 37 & 610.50 & 57 & 940.50 & 77 & 1270.50 & 97 & 1600.50 \\
\hline 18 & 297.00 & 38 & 627.00 & 58 & 957.00 & 78 & 1287.00 & 98 & 1617.00 \\
\hline 19 & 313.50 & 39 & 643.50 & 59 & 973.50 & 79 & 1303.50 & 99 & 1633.50 \\
\hline 20 & 330.00 & 40 & 660.00 & 60 & 990.00 & 80 & 1320.00 & 100 & 1650.00 \\
\hline
\end{tabular}

TABLE XI. LINKS IN FEET, 10THS AND 100THS OF FEET

\begin{tabular}{|c|c|c|c|c|c|c|c|c|c|c|c|}
\hline 苞 & Feet & $\stackrel{\text { m }}{\stackrel{\text { I }}{5}}$ & Feet & 点 & Feet & $\stackrel{5}{5}$ & Feet & $\frac{9}{5}$ & Feet & $\stackrel{2}{\frac{x}{5}}$ & F'eet \\
\hline 1 & 0.66 & 18 & 11.88 & 35 & 23.10 & 52 & 34.32 & 69 & 45.54 & 86 & 56.76 \\
\hline 2 & 1.32 & 19 & 12.54 & 36 & 23.76 & 53 & 34.98 & 70 & 46.20 & 87 & 57.42 \\
\hline 3 & 1.98 & 20 & 13.20 & 37 & 24.42 & 54 & 35.64 & 71 & 46.86 & 88 & 58.08 \\
\hline 4 & 2.64 & 21 & 13.86 & 38 & 25.08 & 55 & 36.30 & 72 & 47.52 & 89 & 58.74 \\
\hline 5 & 3.30 & 22 & 14.52 & 39 & 25.74 & 56 & 36.96 & 73 & 48.18 & 90 & 59.40 \\
\hline 6 & 3.96 & 23 & 15.18 & 40 & 26.40 & 57 & 37.62 & 74 & 48.84 & 91 & 60.06 \\
\hline 7 & 4.62 & 24 & 15.84 & 41 & 27.06 & 58 & 38.28 & 75 & 49.50 & 92 & 60.72 \\
\hline 8 & 5.28 & 25 & 16.50 & 42 & 27.72 & 59 & 38.94 & 76 & 50.16 & 93 & 61.38 \\
\hline 9 & 5.94 & 26 & 17.16 & 43 & 28.38 & 60 & 39.60 & 77 & 50.82 & 94 & 62.04 \\
\hline 10 & 6.60 & 27 & 17.82 & 44 & 29.04 & 61 & 40.26 & 78 & 51.48 & 95 & 62.70 \\
\hline 11 & 7.26 & 28 & 18.48 & 45 & 29.70 & 62 & 40.92 & 79 & 52.14 & 96 & 63.36 \\
\hline 12 & 7.92 & 29 & 19.14 & 46 & 30.36 & 63 & 41.58 & 80 & 52.80 & 97 & 64.02 \\
\hline 13 & 8.58 & 30 & 19.80 & 47 & $: 1.02$ & 64 & 42.24 & 81 & 53.46 & 98 & 64.68 \\
\hline 14 & 9.24 & 31 & 20.46 & 48 & 31.68 & 65 & 42.90 & 82 & 54.12 & 99 & 65.34 \\
\hline 15 & 9.90 & 82 & 21.12 & 49 & 32.34 & 66 & 43.56 & 83 & 54.78 & 100 & 66.00 \\
\hline 16 & 10.56 & 33 & 21.78 & 50 & 33.00 & 67 & 44.22 & 84 & 55.44 & 101 & 66.66 \\
\hline 17 & 11.22 & 34 & 22.44 & 51 & 33.66 & 68 & 44.88 & 85 & 56.10 & 102 & 67.32 \\
\hline
\end{tabular}




\section{Sokkia Surveying Systems, Instruments, Equipment and Supplies}

- Total Stations

-Electronic Field Book

-Mapping Software

-EDM Systems

- Theodolites

- Levels

- Transits

-Tripods

- Rods

-Hand Levels

- Tapes

-Planimeters

-Accessories

The paper in this book is a fine quality thick $50 \%$ rag ledger specially treated during the making to give "High Wet Strength." It retains is strength and writing surfaces when dried after having been subjected to extreme weather conditions. 


\section{FIELD BOOKS}

Rain-resistant fine quality ledger paper, bound in highvisibility durable yellow imitation leather. Printed in waterproof ink.

Stock No. 8152-00 Transit Field Book. Size $4^{1 / 2} \times 7^{1 / 1} / 4$ inches. .

Stock No. 8152-05 Economy Field Book. Spiral bound, Paperback. Size $4 \frac{1}{2} \times 7^{1} / 4$ inches.

Stock No. 8152-10 Economy Field Book. Same as above except saddle stitched (stapled).

Left page blue horizontal lines; red vertical lines.

Right page 4 horizontal and 8 vertical blue lines; red vertical center line.

Stock No. 8152-20 Mining Transit Book. Size $4^{1} / 2 \times 7^{1 / 4}$ inches.

Left page blue horizontal lines; red vertical center line.

Right page $8 \times 8$ blue lines; red vertical lines.

Stock No. 8152-30 Engineers Fìeld Book. Size $4^{1} \frac{1}{2} \times 7^{1 / 1} / 4$ inches.

Left page blue horizontal' lines; red vertical lines.

Right page $10 \times 10$ blue lines; red vertical center line. Inch lines heavy.

Stock No. 8152-50 Level B̈ook Kं Size $4 \times 6^{1} / 2$ inches.

Stock No. 8152-55 Level Book Size 4 $\frac{1}{2} \times 7^{1} / 4$ inches. Both pages blue horizontal lines; red vertical lines. 6 vertical columns.

Stock No. 8152-60 Field Bookt. Size $4^{\frac{1}{1}} / 2 \times 7^{1 / 1} / 4$ inches. Left page blue horizontal lines; red vertical lines.

Right page $4 \times 4$ blue lines; red vertical center line.

Stock No. 8152-75 Cross Section Book. Size $6^{1} / 2 \times 8^{1} / 2$ inches.

Both pages $10 \times 10$ blue lines; inch lines slightly heavier.

Stock No. 8152-80 Duplicating Transit Book. Size $4^{\frac{1}{2} / 2 \mathrm{X}}$ $7 \%$ inches.

Left page blue horizontal lines; red vertical lines.

Right page 4 horizontal and 8 vertical blue lines; red vertical center line. Pages numbered and perforated. Carbon paper. 


\section{CURVE FORMULAE}

$\mathrm{D}=$ Degree of Curve

$1^{\circ}=1$-Degree of Curve

$2^{\circ}=2$-Degree of Curve

P.C. $=$ Point of Curve

P.T. = Point of Tangent

P.I. = Point of Intersection

I = Intersection of Angle, Angle between Two Tangents

$\mathbf{L}=$ Length of Curve, from P.C. to P.T.

$T=$ Tangent Distance

E = External Distance

$\mathbf{R}=$ Radius

L.C. $=$ Length of Chord

$M$ = Length of Middle Ordinate

c = Length of Sub-Chord

$\mathrm{d}=$ Angle of Sub-Chord

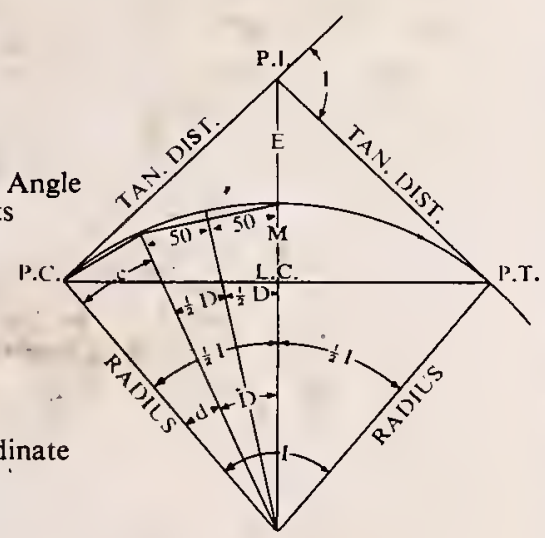

$R=\frac{\text { L.C. }}{2 \sin \frac{1}{2} I} T=R \tan \frac{1}{2} I=\frac{\text { L.C. }}{2 \cos \frac{1}{2} I}$

$\frac{\text { L.C. }}{2}=\mathrm{R} \sin \frac{\mathrm{I}}{2}, \mathrm{D} 1^{\circ}=\mathrm{R}=5730, \mathrm{D} 2^{\circ}=\frac{5730}{2}, \mathrm{D}=\frac{5730}{\mathrm{R}}$

$\mathbf{M}=\mathbf{R}\left(1-\cos \frac{1}{2} \mathrm{I}\right),=\mathrm{R}-\mathrm{R} \cos \frac{\mathrm{I}}{2}$

$\frac{E+R}{R}=\sec \frac{I}{2}, \frac{R-M}{R}=\cos \frac{I}{2}$

$c=2 R \sin \frac{1}{2} d, d=\frac{c}{2 R}$

L.C. $=2 \mathrm{R} \sin \frac{1}{2} \mathrm{I}, \mathrm{E}=\mathrm{R}\left(\sec \frac{1}{2} \mathrm{I}-1\right),=\mathrm{R} \sec \frac{\mathrm{I}}{2}-\mathrm{R}$

Minutes in Decimals of a Degree

\begin{tabular}{|c|c|c|c|c|c|c|c|c|c|c|c|}
\hline $\mathbf{1}^{\prime}$ & .0167 & $\mathbf{1 1}$ & .1833 & $\mathbf{2 1}$ & .3500 & $\mathbf{3 1}$ & .5167 & $41^{\prime}$ & .6833 & $\mathbf{5 1} 1^{\prime}$ & .8500 \\
$\mathbf{2}$ & .0333 & $\mathbf{1 2}$ & .2000 & $\mathbf{2 2}$ & .3667 & $\mathbf{3 2}$ & .5333 & $\mathbf{4 2}$ & .7000 & $\mathbf{5 2}$ & .8667 \\
$\mathbf{3}$ & .0500 & $\mathbf{1 3}$ & .2167 & $\mathbf{2 3}$ & .3833 & $\mathbf{3 3}$ & .5500 & $\mathbf{4 3}$ & .7167 & $\mathbf{5 3}$ & .8833 \\
$\mathbf{4}$ & .0667 & $\mathbf{1 4}$ & .2333 & $\mathbf{2 4}$ & .4000 & $\mathbf{3 4}$ & .5667 & $\mathbf{4 4}$ & .7333 & $\mathbf{5 4}$ & .9000 \\
$\mathbf{5}$ & .0833 & $\mathbf{1 5}$ & .2500 & $\mathbf{2 5}$ & .4167 & $\mathbf{3 5}$ & .5833 & $\mathbf{4 5}$ & .7500 & $\mathbf{5 5}$ & .9167 \\
$\mathbf{6}$ & .1000 & $\mathbf{1 6}$ & .2667 & $\mathbf{2 6}$ & .4333 & $\mathbf{3 6}$ & .6000 & $\mathbf{4 6}$ & .7667 & $\mathbf{5 6}$ & .9333 \\
7 & $\mathbf{1 1 6 7}$ & $\mathbf{1 7}$ & .2833 & 27 & .4500 & $\mathbf{3 7}$ & .6167 & $\mathbf{4 7}$ & .7833 & $\mathbf{5 7}$ & .9500 \\
$\mathbf{8}$ & .1333 & $\mathbf{1 8}$ & .3000 & $\mathbf{2 8}$ & .4667 & $\mathbf{3 8}$ & .6333 & $\mathbf{4 8}$ & .8000 & $\mathbf{5 8}$ & .9667 \\
$\mathbf{9}$ & .1500 & $\mathbf{1 9}$ & .3167 & $\mathbf{2 9}$ & .4833 & $\mathbf{3 9}$ & .6500 & $\mathbf{4 9}$ & .8167 & $\mathbf{5 9}$ & .9833 \\
$\mathbf{1 0}$ & .1667 & $\mathbf{2 0}$ & .3333 & $\mathbf{3 0}$ & .5000 & $\mathbf{4 0}$ & .6667 & $\mathbf{5 0}$ & .8333 & $\mathbf{6 0}$ & $\mathbf{1 . 0 0 0 0}$ \\
\hline
\end{tabular}

Inches in Decimals of a Foot

\begin{tabular}{|c|c|c|c|c|c|c|c|c|c|c|}
\hline$\frac{1}{16}$ & $\frac{3}{32}$ & $\frac{3}{8}$ & $\frac{3}{18}$ & $\frac{1}{5}$ & $\frac{5}{18}$ & $\frac{3}{8}$ & $\frac{1}{2}$ & $\frac{5}{8}$ & $\frac{3}{4}$ & $\frac{7}{8}$ \\
.0052 & .0078 & .0104 & .0156 & .0208 & .0260 & .0313 & .0417 & .0521 & .0625 & .0729 \\
\hline \hline 1 & 2 & 3 & 4 & 5 & 6 & 7 & 8 & 9 & 10 & 11 \\
.0833 & .1667 & .2500 & .3333 & .4167 & .5000 & .5833 & .6667 & .7500 & .8333 & .9167 \\
\hline
\end{tabular}




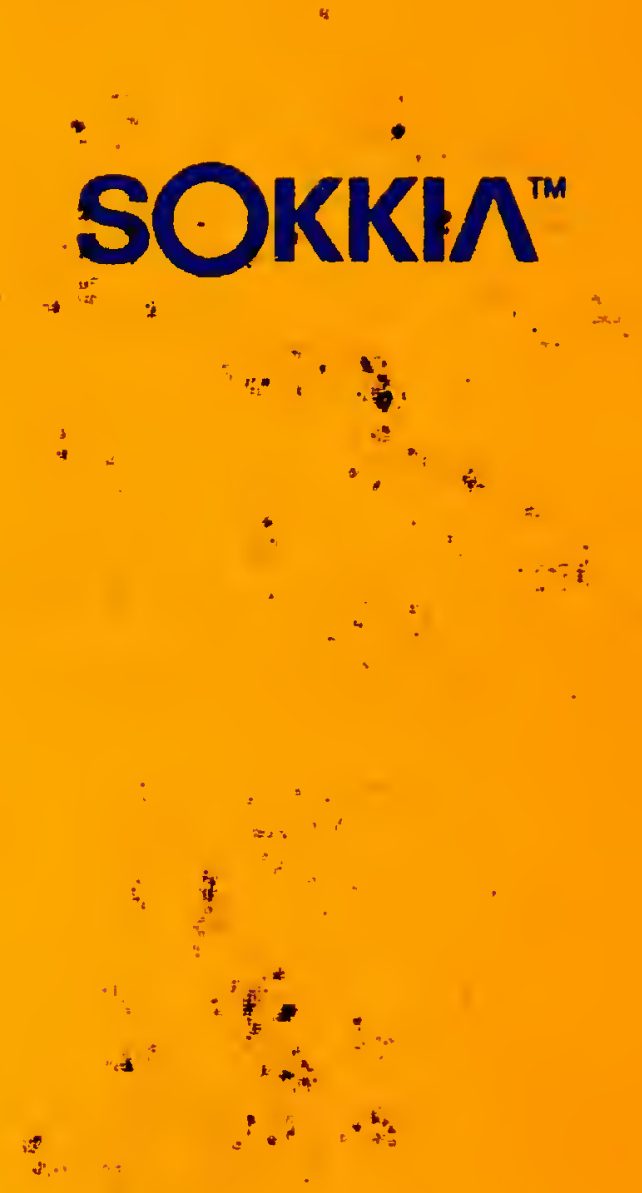

MADE IN U.S.A. 